\title{
STABILIZATION OF HORSERADISH PEROXIDASE USING EPOXY NOVOLAC RESINS FOR APPLICATIONS WITH MICROFLUIDIC PAPER-BASED ANALYTICAL DEVICES
}

\author{
A Thesis \\ presented to \\ the Faculty of California Polytechnic State University, \\ San Luis Obispo \\ In Partial Fulfillment \\ of the Requirements for the Degree \\ Master of Science in Polymers and Coatings Science
}

by

Cory A. Chaplan

June 2014 
(C) 2014

Cory A. Chaplan

ALL RIGHTS RESERVED 
COMMITTEE MEMBERSHIP

TITTLE:

Stabilization of Horseradish Peroxidase using Epoxy

Novolac Resins for Applications with Microfluidic

Paper-Based Analytical Devices

AUTHOR:

Cory A. Chaplan

DATE SUBMITTED:

June 2014

COMMITTEE CHAIR:

Andres W. Martinez, Ph.D.

Assistant Professor of Chemistry and Biochemistry

COMMITTEE MEMBER: Philip J. Costanzo, Ph.D.

Associate Professor of Chemistry and Biochemistry

COMMITTEE MEMBER: Emily J. Fogle, Ph.D.

Assistant Professor of Chemistry and Biochemistry 


\begin{abstract}
Stabilization of Horseradish Peroxidase using Epoxy Novolac Resins for Applications with Microfluidic Paper-Based Analytical Devices
\end{abstract}

Cory A. Chaplan

Microfluidic paper-based analytical devices (microPADs) are an emerging platform for point-of-care diagnostic tests for use by untrained users with potential applications in healthcare, environmental monitoring, and food safety. These devices can be developed for a multitude of different tests, many of which employ enzymes as catalysts. Without specialized treatment, some enzymes tend to lose their activity when stored on microPADs within 48 hours, which is a major hurdle for taking these types of devices out of the laboratory and into the real world. This work focused on the development of simple methods for stabilizing enzymes by applying polymers to chromatography paper. The longterm stabilization was exlored and SU-8 of various concentrations was found to stabilize horseradish peroxidase for times in excess of two weeks. A variety of microPAD fabrications, enzyme dispensing methods, and substrate delivery techniques were explored.

Keywords: Microfluidic Paper-Based Analytical Devices, MicroPADs, Enzyme Stabilization, Epoxy Novolac Resins, SU-8, Horseradish Peroxidase. 


\section{ACKNOWLEDGMENTS}

I would like to believe those reading these acknowledgments are looking here for inspiration, guidance, or knowledge. Believing this, I would first like to thank the reader. By taking the time to read even this sentence, you justify all of my metaphorical (yet perhaps literal) blood, sweat, and tears that have been poured into this project.

As a close second I would like to acknowledge and thank Dr. Andres Martinez. Without your support, guidance, and knowledge, the research I performed would not have an ounce of what I present today. It has been both an honor and a privilege to work under you as a student and scientist.

Drs. Phil Costanzo and Emily Fogle, having been a student under each of you I can happily say I am better off as both a scientist and a human. Dr. Costanzo, your drive, determination, and perseverance towards excellence will remind me constantly to follow my ambitions and never accept anything less than pursuing perfection. Dr. Fogle, your care, precision, and steadfast support will remind me to reflect and grow from my experiences with nothing less than a good light. Thank you both for all of your efforts in helping me become a "Master of Science."

I, of course, must acknowledge my friend, brother, and colleague, Haydn Mitchell. It's hard to believe that we've known each other for over three years, it feels like thirty. But perhaps that's just because of all the 14 hour days in lab. Thank you for everything, I know there's more to come. In addition, Team AWesoMe: you're awesome.

A special thank you to Dr. Lori Robins, had you not allowed me to do research for you all that time ago, I would certainly be in a much different place today. I owe, let's say (roughly) 63\% percent of who and where I am today, to you. For that and for so much more, I cannot thank you enough!

I would be remiss if I did not acknowledge the faculty of the Chemistry \& Biochemistry Department at Cal Poly. To those of you who know me, have supported me, and kept me in line throughout my stay at Cal Poly, I thank you (you know who you are). I would also like to acknowledge the Polymers and Coatings Program and all of those involved, it has been an incredible learning experience - one that I hope many more yet to come can appreciate.

Finally, this project was made possible by funding from $3 \mathrm{M}$. Additional funding was provided by the Extramural Funding Initiative (Cal Poly). 


\section{TABLE OF CONTENTS}

List of Tables $\quad$ ix

List of Figures $\quad$ X

1 Introduction 1

1.1 Microfluidic Paper-Based Analytical Devices . . . . . . . . . . . . . 1

1.1.1 History of Microfluidic Paper-based Analytical Devices . . . . . . 3

1.1.1.1 Photolithography \& Fabricating Microfluidic Paper-Based Analytical Devices .............. . . 4

\subsubsection{Wax Printing \& Fabricating Microfluidic Paper-Based} Analytical Devices . . . . . . . . . . . 10

1.1.2 Current Challenges Facing Microfluidic Paper-Based Analytical

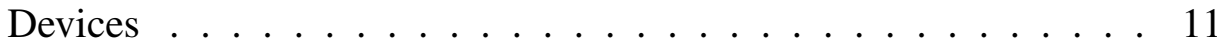

1.2 Enzyme Stabilization . . . . . . . . . . . . . . . 13

1.3 Research Plan . . . . . . . . . . . . . . . . . . . 16

1.3.1 Fabrication ..................... 17

1.3.2 Performance ....................... 17

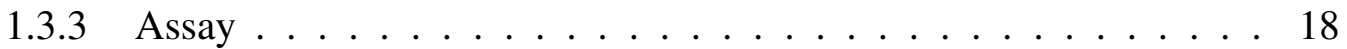

1.3.4 Shelf Life . . . . . . . . . . . . . . . . . . . 18

2 Methods and Materials $\quad 19$

2.1 Materials ............................. 19

2.2 Microfluidic Paper-Based Analytical Device Design, Processing, and Development ............................. 19

2.2.1 Electronic Processing and Physical Creation . . . . . . . . . . 20

2.2.2 Epoxy Novolac Resin Preparation . . . . . . . . . . . . . 20 
2.2.3 Photolithographic Process . . . . . . . . . . . . . . . . 21

2.2.4 Alternative Polymer Matrices . . . . . . . . . . . . . . 23

2.3 Microfluidic Paper-Based Analytical Device Characterization . . . . . 28

2.3.1 Fourier Transform Infrared Spectroscopy _ . . . . . . . . . 28

2.3.2 Microfluidic Paper-Based Analytical Device Hydrophobicity . . . . 28

2.4 Horseradish Peroxidase Assay . . . . . . . . . . . . . . . . . . . . 29

2.4.1 Assay Solution Preparation . . . . . . . . . . . . . . . . 29

2.4.2 Microfluidic Paper-Based Analytical Device Implementation (Shelf Life $\ldots \ldots \ldots \ldots \ldots \ldots \ldots$

2.4.3 Microfluidic Paper-Based Analytical Device Post-Assay Processing 32

3 Results and Discussion $\quad 33$

3.1 Microfluidic Paper-Based Analytical Device Design, Processing, and De-

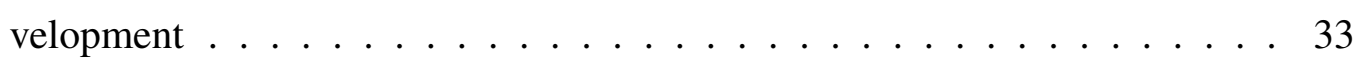

3.1 .1 Photolithographic Process . . . . . . . . . . . . . 33

3.1.1.1 Photoresist application $\ldots \ldots \ldots 33$

3.1 .1 .2 Pre-Bake . . . . . . . . . . . . . . 34

3.1.1.3 Exposure . . . . . . . . . . . . 35

3.1.1.4 Post-Exposure and Hard Bakes . . . . . . . . . . . . . 36

3.1.1.5 Development . . . . . . . . . . . . . . . 37

3.1.2 Alternative Polymer Matrices _ . . . . . . . . . . . 37

3.2 Microfluidic Paper-Based Analytical Device Characterization . . . . . . 38

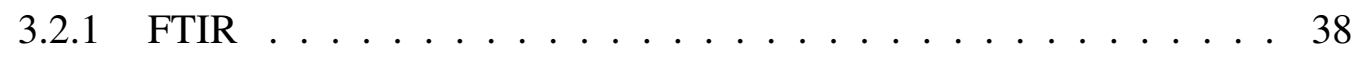

3.2.2 Microfluidic Paper-Based Analytical Device Hydrophobicity . . . . 42

3.3 Enzyme Survival - Microfluidic Paper-Based Analytical Device Shelf Life . 47

3.3.1 Unexposed SU-8 Shelf Life . . . . . . . . . . . . . . . 48

3.3.2 Alternative Matrices Shelf Life . . . . . . . . . . . . . . 49 
3.3.3 SU-X Shelf Life . . . . . . . . . . . . . . . . . . . . . 50

3.3.3.1 Low SU-X Concentrations . . . . . . . . . . . 50

3.3.3.2 High SU-X Concentrations _ . . . . . . . . . . 51

4 Conclusions $\quad 61$

$\begin{array}{ll}\text { References } & 63\end{array}$

$\begin{array}{ll}\text { Appendices } & 68\end{array}$

A Reference FTIR Spectra $\ldots \ldots \ldots \ldots$

B Analytical Methods, $2014,1296 \ldots \ldots \ldots \ldots$. . . . . . . . 71 


\section{LIST OF TABLES}

2.2.1 Theoretical values for SU-X solution preparations. . . . . . . . . . . . 21

2.2.2 Alternate Polymer Matrices Solution Preparation. . . . . . . . . . . 26

2.4.1 Individual microPAD assay conditions, " $\sqrt{\mathrm{s}}$ " indicating reagents used. . . . 30 


\section{LIST OF FIGURES}

1.1.1 One of the first published paper-based analytical devices. . . . . . . . . . 4

1.1.2 Comparison of positive and negative tone resists at the exposure stage of photolithographic processing. . . . . . . . . . . . . 5

1.1.3 Representative cross-section of the photolithographic process used in microPAD fabrication. Reproduced with permission from Wiley. . . . . . . . 9

1.1.4 Representative cross-section of wax printing microPAD fabrication. Reproduced with permission from Wiley. . . . . . . . . . . . . . 10

1.1.5 Comparison of (left) photolithographic and (right) wax print fabricated microPADs. Reproduced with permission from Wiley. . . . . . . . . . . . 11

1.1.6 Stability of microPAD glucose bioassasy over time. Reproduced with permission from the American Chemical Society. . . . . . . . . . . . . 13

1.2.1 Process of protein deactivation in organic solvents where: (1) water is stripped away by water-miscible organic solvents, (2) the partially dehydrated protein binds with the organic solvent, and (3) a conformational transition of the protein into a denatured state. Reproduced with permission from Elsevier. . . . . . . . . . . . . . . . . . 15

2.2.1 A 96-well plate designed for wax patterning, using the same dimensions as a $96-$ well plate. . . . . . . . . . . . . . . . 22

2.2.2 A microPAD impregnated with $40 \%(w / w)$ SU-8 solution in acetone. . . . . 22

2.2.3 Removing excess solution from microPAD . . . . . . . . . . . . 24

2.2.4 "The box" is used as an exposure chamber for SU-X impregnated microPADs: (a) "The box" with a microPAD awaiting exposure. The UV lamp sits completely within the box, and is elevated $4 \mathrm{~cm}$ above the floor of "the box:" (b) the UV lamp in place within "the box," ready for the exposure. 24 
2.2.5 Post and hard bake setup for microPAD. . . . . . . . . . . . . 25

2.2.6 MicroPAD development: (a) acetone bath, and (b) IPA rinse. . . . . . . . 25

2.2.7 Completed microPAD 96-well paper plate. . . . . . . . . . . . . 27

3.2.1 A FTIR spectrum of (bottom) cellulose found in the untreated chromatography paper, and (top) developed (acetone, IPA washed) paper used in the production of microPADs. . . . . . . . . . . . . . . 39

3.2.2 A FTIR spectrum of (bottom) 40\% (w/w) SU-8 impregnated paper before exposure, and (top) after exposure, in open environment. . . . . . . . . 40

3.2.3 A FTIR spectrum of an uncrosslinked portion of 40\% (w/w) SU-8 impregnated paper after exposure, in open environment. . . . . . . . . 41

3.2.4 A FTIR spectrum of $40 \%$ (w/w) SU-8 after the hard bake. . . . . . . . . 43

3.2.5 Stacked FTIR spectra of the various SU-8 concentrations used: (top) $40 \%$ (w/w) SU-8, (middle) 5\% (w/w) SU-8, (bottom) 0\% (w/w) SU-8. . . . . 43

3.2.6 A FTIR spectrum of 40\% (w/w) SU-8 with 4\% (w/w) PS post-development. 46

3.3.1 Solution stabilized horseradish peroxidase, stored at $(\bullet) 4^{\circ} \mathrm{C}$ and $(\square)$ room temperature for (Blue) HRP in 1X PBS and (Red) HRP in IPA. . . . . . . 53

3.3.2 HRP deposited to microPADs: $(\bullet)$ unstabalized HRP on paper, and (汭 trehalose stabilized HRP on paper. . . . . . . . . . . . . . . 54

3.3.3 Shelf life for variable low concentrations of SU-8, both with and without trehalose for unexposed SU-8 without development. . . . . . . . . . . 55 
3.3.4 Composite image of the results displayed in Figure 3.3.3. Odd numbered columns contain $1 \mu \mathrm{L}$ of $1 \mathrm{M}$ trehalose in addition to $2.5 \%$ (w/w) SU-8. Even numbered columns contain no trehalose, but retain the $2.5 \%(\mathrm{w} / \mathrm{w})$ SU-8. Columns $1 \& 2$ represent day 0 results; columns $3 \& 4$ represent day 3; columns $5 \& 6$ represent day 7; columns $7 \& 8$ represent day 10; 9 $\& 10$ represent day 14 ; finally, columns $11 \& 12$ represent day 17 . Rows A through $\mathrm{E}$ include all reagents including enzyme. Rows $\mathrm{F}$ through $\mathrm{H}$ contain all reagents except enzyme. With the exception of column 1, rows F-H which do contain enzyme. . . . . . . . . . . . . . . . 55

3.3.5 MicroPADs containing (Blue) 2.5\% (w/w) SU-8, (Red) 2.5\% (w/w) PS MW 800-5000, and (Green) 2.5\% (w/w) NC both (-) presoaked with DI H2O before enzyme deposition, and (ם) enzyme deposited directly after

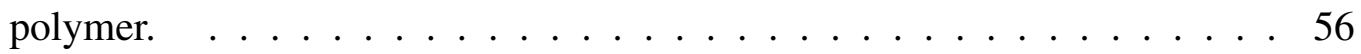

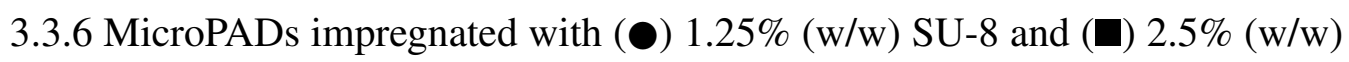
impregnated with (Red) IPA-HRP and (Blue) 1X PBS-HRP. Assays were run with $50 \%(\mathrm{v} / \mathrm{v})$ IPA-ABTS. . . . . . . . . . . . . . 57

3.3.7 Summary of low SU-8 concentrations performed over the course of the shelf life experiments. . . . . . . . . . . . . . 58

3.3.8 Hydrophobic concentrations of SU-X on fully processed microPADs. Assays were run with $50 \%(\mathrm{v} / \mathrm{v})$ IPA-ABTS to avoid the hydrophobic effects of high SU-X concentrations: (a) microPADs with fully processed (๑)10\% $(\mathrm{w} / \mathrm{w}),(\mathbf{\square}) 20 \%(\mathrm{w} / \mathrm{w})$, and $(\diamond) 30 \%(\mathrm{w} / \mathrm{w})$ "SU-4;" and (b) microPADs with fully processed (๑)10\% (w/w), (ם) $20 \%(\mathrm{w} / \mathrm{w})$, and (•) $30 \%(\mathrm{w} / \mathrm{w})$ "SU-8." ............................ 59

3.3.9 Comparison of activity of high SU-X concentration microPADs spotted with (a) IPA-HRP, or (b) 1X PBS-HRP. . . . . . . . . . . . . . . 60

A.1 FTIR spectrum of acetone. . . . . . . . . . . . . . . 69 
A.2 FTIR spectrum of 2-propanol. . . . . . . . . . . . . 70 


\section{LIST OF SCHEMES}

1 Photoacid generation from triarylsulfonium hexafluorophosphate salts. . . . 7

2 Photoacid initiation and propagation to the monomer SU-8. . . . . . . . 8

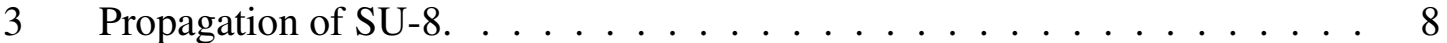

4 Termination of SU-8 by chain transfer to an alcohol or water $\left(\mathrm{R}_{3}=\mathrm{H}\right)$. $\ldots \quad$. 8

5 ABTS oxidation is catalyzed by horseradish peroxidase in the presence of a half equivalent of hydrogen peroxide producing a radical ion. . . . . . . . 18 


\section{Introduction}

Imagine taking a laboratory away from its traditional, white lab coat, controlled environment and moving it to a remote desert in Africa, a favela in Brazil, or the side of a busy freeway in Los Angeles. It is not difficult to argue that due to the relatively harsh environment, these laboratories would be prone to equipment failure, loss of accuracy and precision, and costly maintenance and upkeep, which would likely not be practical in any of these given locations. Laboratory personnel is another issue all together. Imagine trying to staff a laboratory in a remote desert in Africa, a favela in Brazil or the side of a busy freeway in Los Angeles. It would be virtually impossible to find people in these locations that already possessed the specialized skills required to operate the laboratory instruments, and it would take a significant amount of time to train inexperienced users to perform complex laboratory procedures. And yet, the information that could be obtained by running analytical tests in the field or in remote settings could have a huge positive impact on the well-being of populations in these locations. In summary, there is a strong need for simple diagnostic tests that could be performed in remote settings by untrained users that is not being addressed by current centralized laboratories. One way to address this need would be to develop small, portable, inexpensive and easy-to-use diagnostic tests that could be taken to remote locations and used by untrained users to test for the presence of specific analytes. These devices would have to operate without relying on external equipment or power sources and would have to be stable at room temperature for extended periods of time.

\subsection{Microfluidic Paper-Based Analytical Devices}

Microfluidic paper-based analytical devices, also known as microPADs or 2D paper networks, provide a powerful diagnostic platform for detecting and diagnosing disease and illness, particularly in areas of the world without access to expensive analytical equipment 
or even basic infrastructure. ${ }^{1}$ In order to provide high quality diagnostic products for use in resource-limited settings, the World Health Organization (WHO) has called for the development of diagnostic devices that are A.S.S.U.R.E.D.: affordable, sensitive, specific, user-friendly, rapid and robust, equipment free, and deliverable to end-users. ${ }^{2}$ Paper is an intriguing platform for A.S.S.U.R.E.D. analytical device development for a variety of reasons: (i) paper is readily available and inexpensive even for high quality chromatography paper (Whatman ${ }^{\circledR} 1 \mathrm{chr}: \$ 8.20 / \mathrm{m}^{2}$ from Sigma Aldrich); (ii) paper passively transports aqueous solutions by wicking; (iii) paper is lightweight, allowing it to be transported cheaply and efficiently; (iv) biological samples are near-ubiquitously compatible with cellulose, and cellulose composites, which is the main (if not only) component of paper; ${ }^{3}$ (v) paper can be modified chemically in order to permanently bind proteins, DNA, and small molecules; ${ }^{4,5}$ (vi) paper is easily processed and compatible with a variety of printing technologies; ${ }^{4,6}$ and (vii) paper is flammable, so microPADs can be safely disposed of by incineration.

MicroPADs can be described as point-of-care (POC) diagnostics. ${ }^{7,8,9,10}$ POC testing, also known as patient or bedside testing, encompass a range of techniques with the goal of delivering quick results that lead to improved patient care and treatment without the need for a clinical lab. ${ }^{7,11}$ Early POC diagnostics utilized tablets containing reagents to test glucose levels in patients. ${ }^{11}$ This was followed by the development of dip stick devices and eventually lateral flow tests. ${ }^{11}$ Such devices could be used to test for pregnancy, HIV-1, and cardiac disease (among other conditions). ${ }^{11}$ Today, POC tests are developed for location-specific settings including in a physicians office, for first responders (EMTs, police, firefighters) for emergency care, at home, or by the military. ${ }^{9}$ As a platform for POC tests, paper has been used extensively (see Section .1.1.1). ${ }^{8}$ POC tests for diseases such as malaria, tuberculosis, HIV, and various sexually transmitted infections (gonorrhea, syphilis, chlamydia) among a plethora of ailments could benefit greatly from any POC test, but a POC test developed on paper has the added benefit of more likely meeting the 
A.S.S.U.R.E.D. standard set by the WHO.

From a global health perspective, microPADs have a high potential for meeting the A.S.S.U.R.E.D. standard. Unfortunately, a large hurdle remains to be addressed which entails interpretation of diagnostic test's results; ${ }^{12,8}$ this hurdle could potentially be surpassed by employing microPAD tests that can be imaged via telemedicine. ${ }^{13,8}$ Telemedicine is broadly defined as the use of information and communication technology to exchange health care services between individuals and medical professionals or trained medical technicians, without the need for a face-to-face meetings. ${ }^{14}$ Combined with the appropriate diagnostic test and portable imaging equipment, an image could easily be sent from endusers to a staffed medical center for rapid analysis and feedback from nearly anywhere in the world (given the appropriate infrastructure is in place).

\subsubsection{History of Microfluidic Paper-based Analytical Devices}

The use of paper-based analytical devices and techniques have been recorded as early as 23 to 79 C.E. with Pliny detecting ferrous sulfate in verdigris with paper saturated with a gallnuts extract. ${ }^{15}$ Much later, in 1937, Yagoda published work detailing the fabrication of filter paper impregnated with paraffin to create confined test zones for qualitative metal ion assays. ${ }^{15}$ In this article, Yagoda concludes with: "this [technique] should also prove useful in biological analyses of the composition of blood and urine," foreshadowing the current era of paper-based analytical devices with eerie accuracy. Then in 1949, Müller and Clegg developed a method of preferential elution of a mixture of pigments on filter paper with dye pressed paraffin channels (Figure 1.1.1). ${ }^{16}$ Finally, in 2007, the Whitesides Group at Harvard University introduced and popularized microfluidic paper-based analytical devices (microPADs) as a platform for diagnostic testing in less-industrialized countries, in the field, or as an inexpensive alternative to more advanced technologies used in clinical settings ushering in the current era of microPADs. ${ }^{17}$ Since then, a multitude of techniques have been developed to fabricate hydrophobic barriers around hydrophilic paper, including 


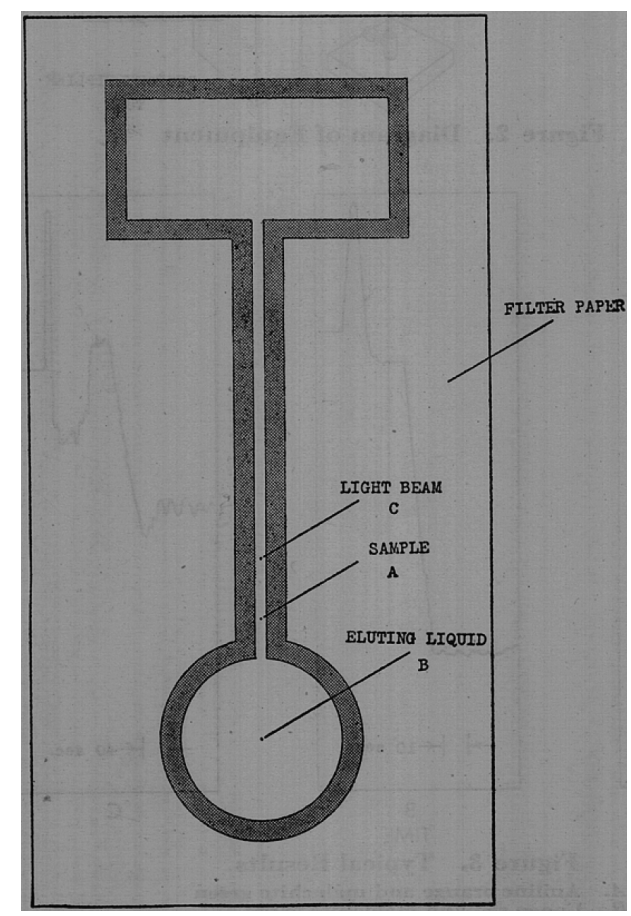

Figure 1.1.1.: One of the first published paper-based analytical devices. ${ }^{16}$

photolithography, plotting with an analogue plotter, ink jet etching, plasma treatment, paper cutting, wax printing, ink jet printing, flexography printing, screen printing, and laser treatment. ${ }^{18}$ Of these, photolithography and wax printing are of special note and are discussed in Sections 1.1.1.1 and 1.1.1.2 respectively.

\subsubsection{Photolithography \& Fabricating Microfluidic Paper-Based Analytical Devices}

Microfluidics have a long history with photolithography, in addition it was the first method introduced by the Whitesides group to pattern paper. ${ }^{17}$ Photolithography employs photoresists, which are light sensitive materials used in industrial processes: poly(methyl methacrylate), poly(methyl glutarimide), phenol formaldehyde resin, and SU-8 are all common resists. SU-8 is one of the most commonly used resists since its introduction by IBM in $1989 .{ }^{19}$ SU-8 falls under the category of negative tone resists, meaning that upon exposure to a radiation source, the material becomes insoluble while unexposed material remains soluble to the solvent used in the development process (Figure 1.1.2) SU-8 solutions have several main components: 1) a bisphenol A novolac epoxy oligomer, 


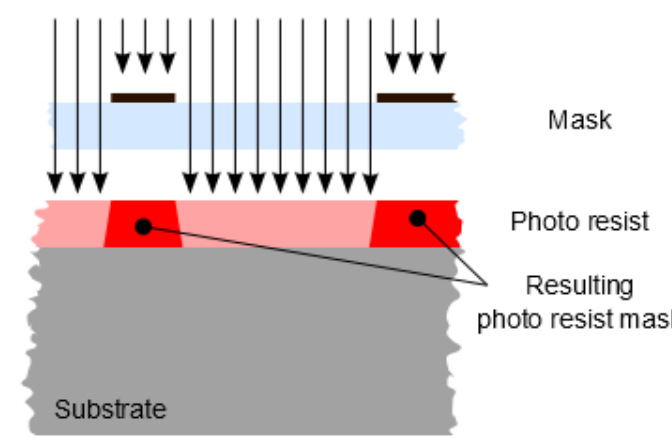

Positive tone resist

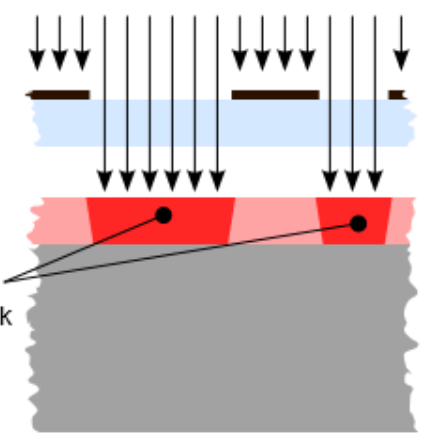

Negative tone resist

Figure 1.1.2.: Comparison of positive and negative tone resists at the exposure stage of photolithographic processing. ${ }^{20}$

2) up to $10 \%$ weight triaryl sulfonium salts, and 3) an appropriate solvent such as propylene glycol monomethyl ether acetate, cyclopentanone, or $\gamma$-butyrolactone. ${ }^{21}$

SU-8 sets itself apart from other photoresist agents due to its ability to produce high aspect ratio structures through relatively simple fabrication. A technique known as L.I.G.A. or lithographie, galvanoformung, abformung, is a multi-step process in which a photoresist goes through lithography, electroplating, and molding. ${ }^{22,23}$ When L.I.G.A. is applied to SU-8, the structures produced can have aspect ratios greater than 1000, but this can be both an expensive and time consuming process. ${ }^{24}$ More commonly used with SU-8 is photolithographic processing. By itself, photolithography can produce structures with an aspect ratio of greater than 20, which compared to a complete L.I.G.A. process is quite poor. However, compared to aspect ratios produced from other photoresist (often less than five) it is clear why SU-8 is the preferred resist for a variety of applications. With other photoresists, issues have arisen with producing high-aspect-ratio, and thick microstructures. These issues are due to minimal penetration of photons through the materials, typical positive photoresists can only achieve thicknesses of tens of $\mu m .^{25,24}$

The fabrication processing parameters involved with the photolithography of SU-8 can be considerably optimized to achieve the best microstructures. ${ }^{23}$ This optimization depends, to some extent, on the application SU-8 is being used for; but can be generalized to 
include five to six steps: 1) coating a substrate, 2) soft bake, 3) exposure, 4) post bake, 5) development, and an optional 6) hard bake. To produce uniform structures, a coating process, which can apply a uniform layer of SU-8, is needed. For nearly all applications and SU-8 formulations, spin coating is used to produce uniform film thicknesses between 0.5 and $200 \mu \mathrm{m}$. After a substrate is coated a soft bake is typically performed in order to vaporize and remove the solvent and increase the density of the film. For SU-8, the ideal temperature to perform a soft bake is approximately $65{ }^{\circ} \mathrm{C} .{ }^{26}$ At this temperature stress due to thermal mismatch between SU-8 and the substrate is minimized producing crackfree structures. The substrate is then ready to be exposed; the most common method of exposure employs near ultraviolet (UV) light on the order of $350-400 \mathrm{~nm}$. Wavelengths higher than $400 \mathrm{~nm}$ are not absorbed by SU-8, yet wavelengths lower than $350 \mathrm{~nm}$ produce significantly lower resolution structures than those produced in the $350-400 \mathrm{~nm}$ range. ${ }^{26}$ Post-exposure baking continues the polymerization process selectively for the exposed portion of the substrate. The post bake has the potential to introduce stress and cracking within the SU-8 structures, which can be avoided by controlling the ramp heat and cooling, total bake time, and the temperature conditions. ${ }^{26}$ To remove the soluble (uncrosslinked) SU-8, the substrate must be developed in an appropriate solvent such as ethyl lactate, diacetone alcohol, or even acetone. Finally, what is referred to as a hard bake may be performed, if the application employs SU-8 as a final structure, in order to completely cure the SU-8.

Diglycidyl ether bisphenol A photoresists (SU-8 and SU-X, a mixture of SU-8 and SU-2.5 to achieve " $\mathrm{X}$ " functionality) have been extensively used and characterized in microelectromechanical systems (MEMS). ${ }^{19,21,22,23,24,25,27}$ Beyond the semiconductor industry, SU-8 has also been developed for biomedical applications as Biomedical MEMS as well. ${ }^{28,29,30}$ This is due its ease of fabrication, thermal and chemical stability, optical transparency, and biological compatibility. ${ }^{28}$

The exposure process begins the polymerization and crosslinking of the photoresist. 
Scheme 1: Photoacid generation from triarylsulfonium hexafluorophosphate salts. ${ }^{31}$

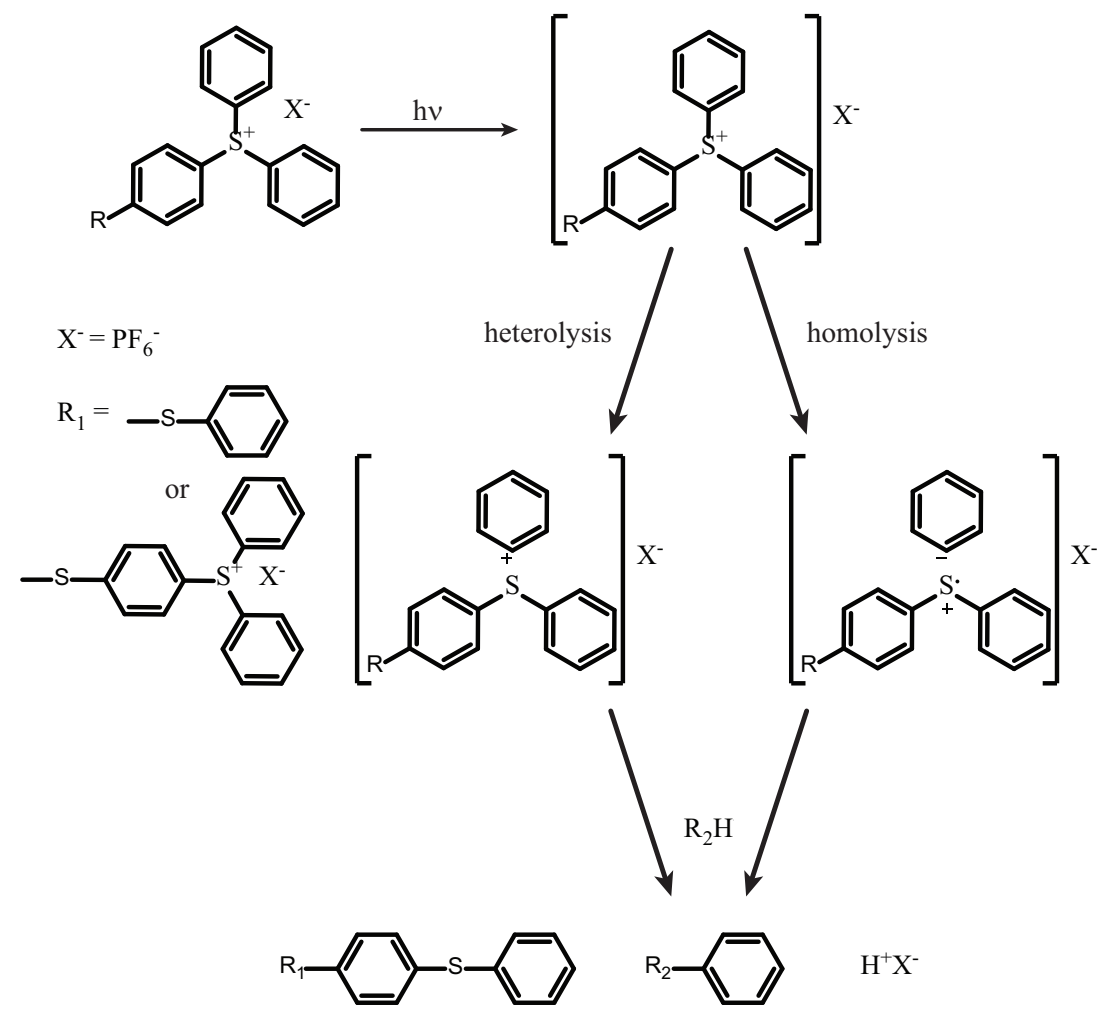

Schemes 1 - 4 follow the photoacid generation, photoinitiation, polymerization initiation, propagation, and termination of the crosslinking of all SU-X photoresists. The photoinitiation in Scheme 1 uses UV light to heterolytically or homolytically cleave the photoinitiation yielding a photoacid. ${ }^{31,32,33}$ This photoacid can then initiate and propagate by cationic chain growth, as shown in Scheme 2 and 3 respectively. Finally, termination can be reached through combination with a counter-ion, beta-proton transfer, or, most likely, chain transfer from the development with isopropyl alcohol. ${ }^{31}$

Fabrication of microPADs historically use a very similar fabrication method. Unlike other photolithographic methods, photolithographic fabrication of microPADs does not build three-dimensional structures onto a substrate. Instead, photolithographic fabrication of microPADs polymerizes a photoresist into the cellulose matrix of paper creating hydrophobic barriers of polymer that direct the capillary action (wicking) of hydrophilic paper channels. Figure 1.1.3 illustrates the fabrication process of the first microPADs 
Scheme 2: Photoacid initiation and propagation to the monomer SU-8.

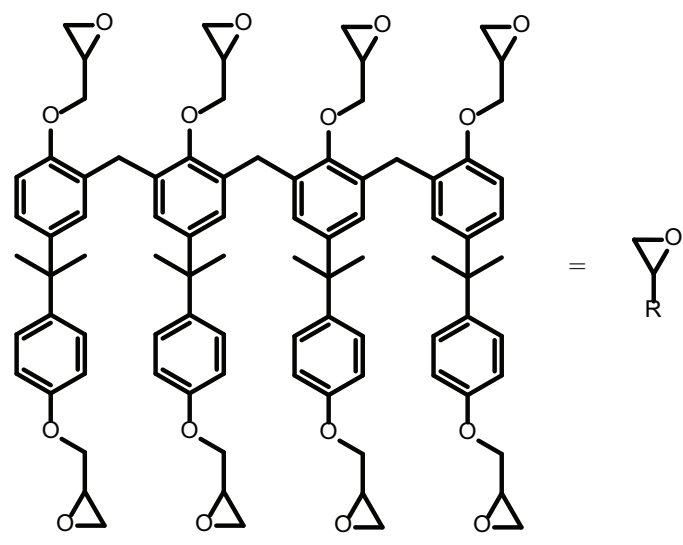

SU-8

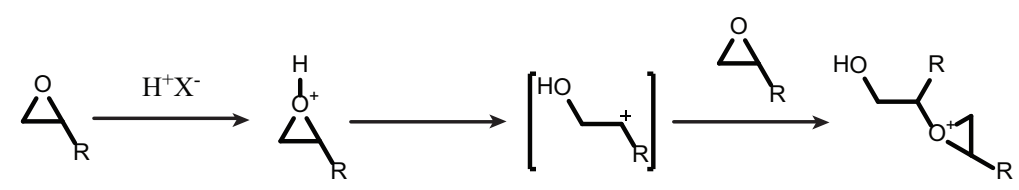

Scheme 3: Propagation of SU-8.

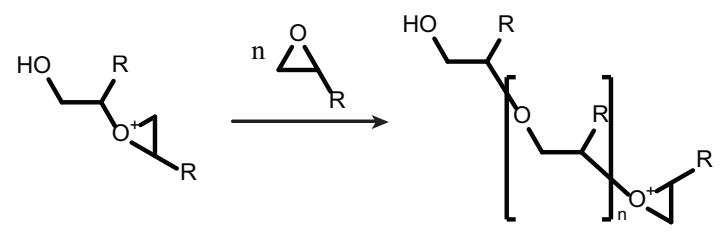

Scheme 4: Termination of SU-8 by chain transfer to an alcohol or water $\left(R_{3}=H\right)$.

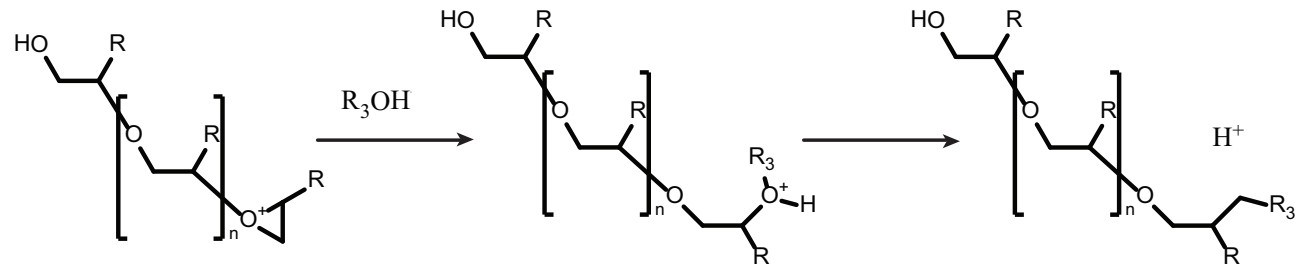




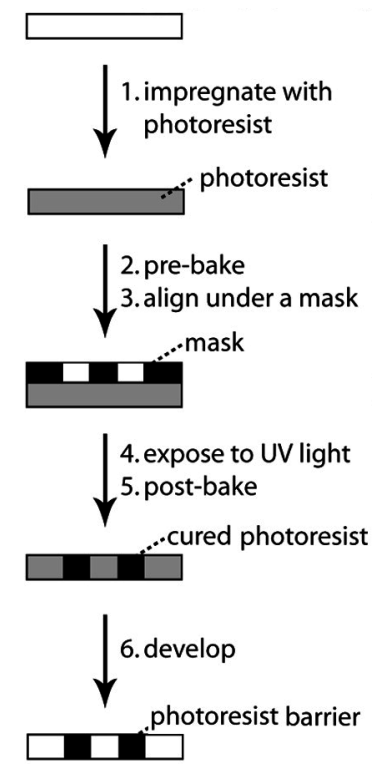

Figure 1.1.3.: Representative cross-section of the photolithographic process used in microPAD fabrication. ${ }^{17}$ Reproduced with permission from Wiley.

produced with photolithography on paper. The most apparent difference is the application of the photoresist to the substrate. With a typical photolithographic fabrication on a silicon wafer, application of the photoresist is done by spin-coating to create a photoresist film of uniform thickness. With microPAD fabrication, the paper must be impregnated with the photoresist. This is because the hydrophobic barrier must penetrate the thickness of the paper to completely prevent flow outside of the barriers. Since this process fills the pores in the paper, it has the effect of increasing the brittleness of the paper making the microPAD vulnerable to bending. ${ }^{34}$ Treatment of the paper with organic solvents to remove uncrosslinked photoresist is another disadvantage to this technique, as a layer of hydrophobic organic residue can often be left on the surface of the paper. ${ }^{17}$ Photolithography is an involved, multi-step, process which is not easily scalable; additionally, photolithography has a relatively high cost compared to other microPAD fabrication methods such as wax printing, and can add undesirable characteristics to the paper (e.g. bending from internal stress of polymerization). ${ }^{34}$ 


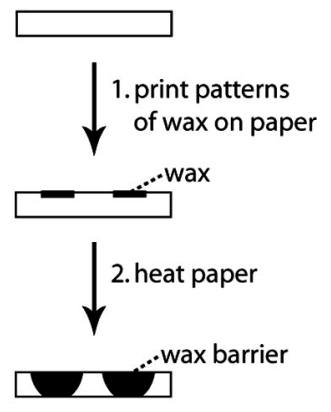

Figure 1.1.4.: Representative cross-section of wax printing microPAD fabrication. ${ }^{17}$ Reproduced with permission from Wiley.

\subsubsection{Wax Printing \& Fabricating Microfluidic Paper-Based Analytical Devices}

Fabrication of microfluidic paper-based analytical devices (microPADs) with wax has numerous advantages, slightly dependent on the method of patterning. Wax screen printing, dipping, and printing are distinct methods of delivering wax to a paper platform. ${ }^{34}$ Of these, wax printing is the most robust method, allowing high throughput and reproducibility between batches ${ }^{34}$ Wax printing was first described by Lu et al., ${ }^{35}$ and concurrently characterized by Carrilho et al. in $2009 .{ }^{36}$ In all cases the processing of wax on paper remains very similar. Figure 1.1 .4 outlines the process of wax fabrication via printing. This process is as simple as heating low-melting point solid wax, patterning the liquid wax onto paper, and heating the paper impregnated with wax to allow the wax to flow through the matrix of the cellulose to the back of the paper. ${ }^{35,36}$ Comparing this approach to photolithography (Figure 1.1.3) it is easy to imagine there is a significant increase in device throughput and prototyping as fabrication time goes down from hours to minutes. Wax impregnation is not without its faults. Barrier resolution is significantly lost when compared to photolithographic fabrication (Figure 1.1.5), ${ }^{17}$ and the combination of high ambient temperatures and long storage can cause the wax to re-melt - potentially making the device unusable. 

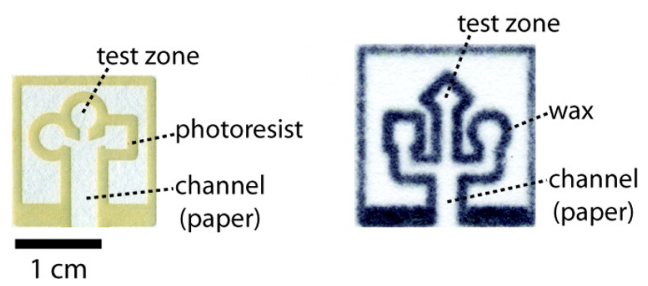

Figure 1.1.5.: Comparison of (left) photolithographic and (right) wax print fabricated microPADs. ${ }^{17}$ Reproduced with permission from Wiley.

\subsubsection{Current Challenges Facing Microfluidic Paper-Based Analytical Devices}

Several challenges face the widespread implementation of microfluidic paper-based analytical devices (microPADs). Those challenges include both device limitations as well as market entry and commercialization of the microPADs. Historical challenges with microPAD limitations include creating fluid channels in paper that provide fixed volumes of sample to the assay reagents within the device, timers, and power sources. ${ }^{34,18}$

The introduction of switches and valves to microPAD design can allow, for example, multi-step protocols that require timed reagent delivery. ${ }^{34}$ A three-dimensional microPAD design can be constructed in a way that allows for an on-valve based on a close-the-gap concept via a simple button. ${ }^{37}$ Yet producing an on-off valve to control sample delivery has yet to be seen in the literature with microPADs.

Fluid mixing remains a challenge with microPADs; passive mixing by capillary action is dominated by diffusion and is often not seen on short-channel microPADs due to laminar flow. It is possible to introduce eddies through the introduction of threads or bypass-able channel blocks, but these can increase the run-times of the devices to an unreasonable extent. ${ }^{34}$

Detection techniques are yet another issue that plague microfluidics as a whole. ${ }^{18}$ Ideally, microPADs would have self-contained readouts displaying the results of the assay. However, this is not always possible due to the nature of the performed assays. Various electrochemical detection methods have been reported and work remarkably well in terms of sensitivity and specificity; the downside of electrochemical detection is that it requires 
additional detection instruments and increases the cost of the test both monetarily and in terms of the A.S.S.U.R.E.D. criterion. ${ }^{34}$ Colorimetric detection is another highly regarded method for microPAD readouts; here too are a variety of problems, such as background noise from the paper or sample and the heterogeneous distribution of colored assay products. The latter is often referred to as the "coffee-ring" effect where soluble assay products flow to, and build up around, the hydrophobic barriers on the edges of the device. These coffee rings make quantitative comparisons difficult, if not impossible. And even when the coffee-ring effect is not present, quantifying results requires a calibration chart, and a handheld reader or a camera phone. ${ }^{13,34,38}$ Preventing the coffee-ring effect depends on the requirements of each assay; however, using substrates whose assay products precipitate out of aqueous solution is a one possible way to prevent a coffee-ring effect. The camera phone plays into the concept of telemedicine but has the issue of lighting conditions affecting the intensities of recorded colors on a device. ${ }^{13,38}$ This can either be addressed by making the lighting and camera conditions under which the images are taken constant, by introducing an external standard, or by using a methodology such as standard-addition. ${ }^{39}$

A final and important challenge facing microPADs involves the ability (or lack thereof) of paper to stabilize assay reagents (particularly enzymes) long-term, especially for quantitative tests. ${ }^{13}$ Figure 1.1.6 gives the first study of enzymatic shelf life on microPADs. In this figure a significant decrease in signal intensity over a relatively short period of time is seen for both unstabilized enzyme (15 days), and trehalose stabilized enzyme (two months). ${ }^{13}$ While, these results indicate a potentially commercially relevant storage and transport time - room for improvement remains, which could be achieved by a variety of physical and chemical means. 


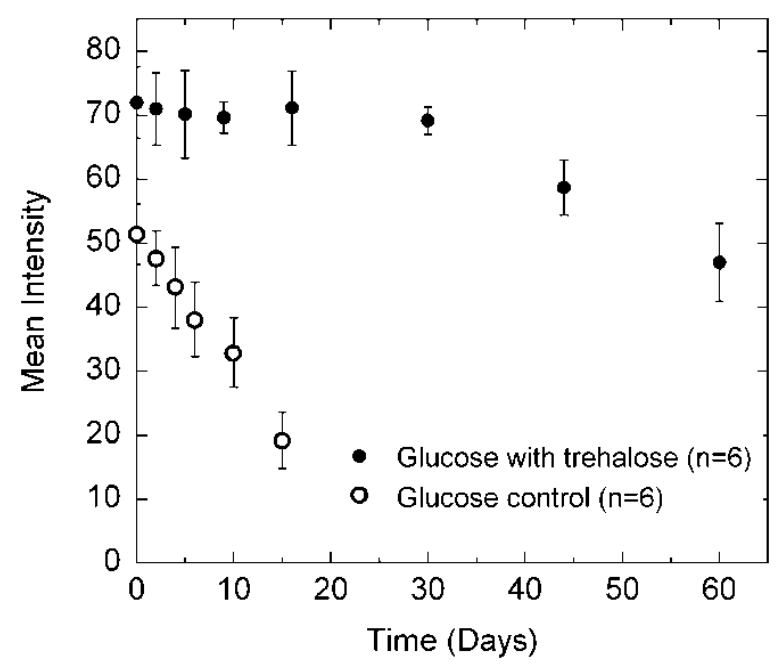

Figure 1.1.6.: Stability of microPAD glucose bioassasy over time. ${ }^{13}$ Reproduced with permission from the American Chemical Society.

\subsection{Enzyme Stabilization}

Enzymes, among other reagents, are essential components of many quantitative and qualitative bioassays. As catalysts, enzymes are unrivaled in their high specificity (both molecular and chiral), and activity; yet they also display inherent instability outside of solution and high costs of production, processing and storage. ${ }^{40}$ Enzyme denaturation, the process of an enzyme unfolding into an inactive state may occur during production, storage, or even at the application stage of the device. Theoretically, denaturation alone is a reversible process, so long as the denatured enzyme does not undergo a chemical or physical change that leads to a permanent loss of activity. ${ }^{40}$ Empirically, many enzymes do not regain full activity once denatured. It is important to recognize the two separate phenomena (temporary and permanent denaturation) and how they may lead to enzyme inactivation. The first is thermodynamic - or conformational - stability. Thermodynamic stability is a measure of an enzyme's conformational resistance to denaturing. ${ }^{40}$ The second is long-term - or kinetic - stability. Kinetic stability refers to an enzyme's resistance to becoming irreversibly inactivated after denaturing. ${ }^{40}$ 
Before discussing methods of stabilization, the variables that may affect a protein's spatial configuration and activity should be noted. These include temperature, $\mathrm{pH}$, chemicals, autolysis, and ionic strength. ${ }^{40}$ In the context of microPADs, the problem of enzyme denaturation leading to permanent loss of enzyme activity is a seemingly surmountable hurdle. Cellulose alone offers functionality to chemically attach proteins. ${ }^{41,42}$ Physical modifications such as the application of the sugar trehalose to microPADs has been shown previously to improve the stability of enzymes stored on these devices. ${ }^{13}$ A variety of enzyme stabilization techniques have been previously presented, each with their own particular advantages and disadvantages. For example: (1) use of extremophile enzyme variants; ${ }^{43}$ (2) genetic modification of organisms to produce more stable enzymes; ${ }^{44}$ (3) chemical modification of enzymes; ${ }^{45}$ (4) the addition of salts, sugars, polyols, or other additives; ${ }^{40}$ and (5) enzyme immobilization to solid carriers. ${ }^{41}$ The most appealing of the above is immobilization which - in addition to minimizing denaturing - also has the benefit of decreasing inhibition by reaction products, selectivity towards non-natural substrates, and better functional properties. ${ }^{41,42}$ Immobilization may be achieved through either non-covalent adsorption, covalent binding, entrapment, or enzyme aggregation/cross-linking. ${ }^{40,41}$ No matter the method of immobilization, the stability of an immobilized enzyme is dictated by the number and type of bonds between the carrier and the protein, the degree of movement available to the protein once immobilized, the carrier environment, and the immobilization conditions. $^{28}$

Of the above immobilization methods, two stand out with respect to microPADs: adsorption/deposition and encapsulation. These two methods have the potential to minimize chemical modifications to the paper, leaving microPADs as robust as possible with respect to the variety of tests which can be performed. In non-covalent adsorption and deposition, proteins can interact with a hydrophobic platform via van der Waals forces and entropy changes; similarly, hydrophilic platforms can interact by hydrogen bonding. ${ }^{41}$

Immobilizing a protein via adsorption has the benefit of not requiring pre-treatment or 


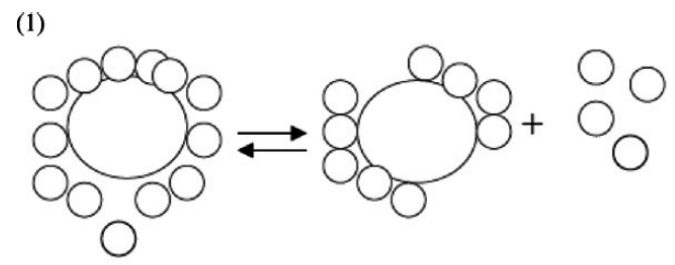

(2)

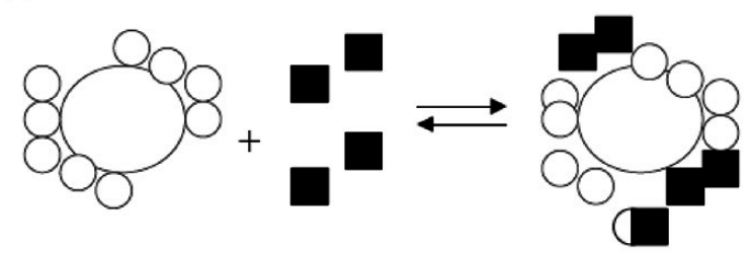

(3)

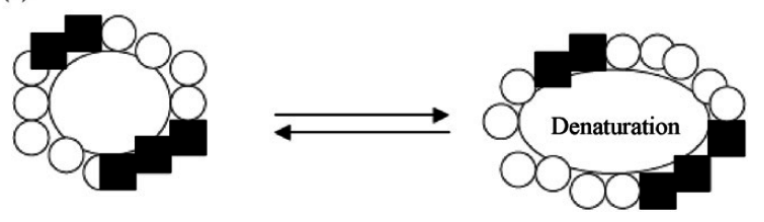

Figure 1.2.1.: Process of protein deactivation in organic solvents where: (1) water is stripped away by water-miscible organic solvents, (2) the partially dehydrated protein binds with the organic solvent, and (3) a conformational transition of the protein into a denatured state. ${ }^{40}$ Reproduced with permission from Elsevier.

chemical modification of the microPAD. ${ }^{41}$ Deposition on the other hand has no hydrophobic or entropic driving force, the deposited protein is immobilized by the evaporation of the aqueous solution. ${ }^{41}$ This could prove problematic and contribute to the coffee-ring effect described in Section 1.1.2, or may assist with devices utilizing a sequential addition of reagents. Organic solvents can aid in the immobilization of proteins in cases when a hydrophobic carrier is used. However, because most proteins contain a hydration sphere around their surface which maintains their three dimensional structure and activity, organic solvents can deactivate or denature proteins based on the hydrophobicity and the solvation capacity of the solvent. Figure 1.2.1 outlines this process of hydration sphere replacement and ultimate enzyme deactivation.

Encapsulation can be achieved by delivering the protein or protein aggregates to a crosslinked carrier, or by crosslinking the carrier around the enzyme to achieve encapsulation. ${ }^{28} \mathrm{Re}-$ gardless of the method of entrapment, this immobilization method offers higher volume 
activity and higher mechanical stability compared to other methods. ${ }^{28}$

\subsection{Research Plan}

The purpose of this project was to characterize the effect of SU-8 on the activity and shelf life of enzymes deposited on microPADs. The motivation for this project came rather unexpectedly while performing enzyme-based glucose assays on microPADs. On the first day of the experiments, a fresh reagent solution containing the enzymes for the assay and trehalose, which was added to stabilize the enzymes based on published results, was added to and dried on the microPAD. Some of the tests were conducted immediately after the device was prepared by adding glucose solutions to the device, and these tests produced a strong signal for the assay. Other devices were stored overnight and the tests were conducted the following day. These devices produced a very weak signal for the assay. After running a series of control tests, we concluded that the reason the signal dropped on the second day was because the enzymes were losing activity when stored on the microPAD. This result was unexpected because previously published work showed that trehalose-stabilized enzymes could survive on microPADs for 30 days with no loss in activity (Figure 1.1.6). It was suspected that the method used to fabricate the devices had an effect on the stability of the enzymes stored on the devices. The devices used for the published study were patterned by photolithography using SU-8. The devices currently being used were patterned by wax printing. This lead to the hypothesis that when devices were patterned using SU-8, a small amount of the polymer remained on the device and contributed to the added enzyme stabilization.

The project was carried out in a series of steps. First, a fabrication procedure allowing for the combination of wax printing and photolithography was optimized. Then methods were developed to characterize the devices that contained SU8. This was followed by an optimization of an assay for horseradish peroxidase to study the enzyme stability, and finally, the stability of HRP on microPADs containing SU8 was studied. 


\subsubsection{Fabrication}

In order to produce a functional microPAD to test the enzyme shelf life on paper, two fabrication techniques are employed: wax printing, and photolithography. The ideal microPAD for testing would use wax to create hydrophobic barriers which would contain SU-8. Reagents could then be delivered to the SU-8 impregnated test zones and the assays performed. For ease of fabrication, wax was impregnated into paper, and heated through. Photolithography could then be performed to deliver photoresist to the device. However, certain considerations needed to be taken into account when performing photolithography in a lab lacking dedicated photolithographic equipment.

Ideally microPAD fabrication would consist of less steps than outlined above in Section 1.1.1.1 and in all be less time consuming than a structural SU-8 MEMS fabrication because there is no need to produce well defined structures typical of SU-8 applications. To expedite this fabrication, initially, a vacuum assisted resin transfer molding process (VARTM) was attempted. VARTM is useful for molding viscous resins, and would potentially be a useful, consistent technique when applied to microPAD fabrication. ${ }^{46}$ Should VARTM prove unsuccessful, photoresist impregnation would follow literature guidelines. ${ }^{13}$ Exposure, due to lack of equipment, will initially be performed in sunlight. This had the benefit of highlighting the robust nature of photopolymerization, yet to better characterize and produce more consistent microPADs a lamp emitting 360 to 400 nm light was also used.

\subsubsection{Performance}

In order to evaluate the ability of the microPAD to perform functionally, it must be characterized in several ways: (1) the hydrophobic effect of the coating, (2) the total delivered, and remaining photoresist after fabrication, and (3) the extent of crosslinking. 
Scheme 5: ABTS oxidation is catalyzed by horseradish peroxidase in the presence of a half equivalent of hydrogen peroxide producing a radical ion.
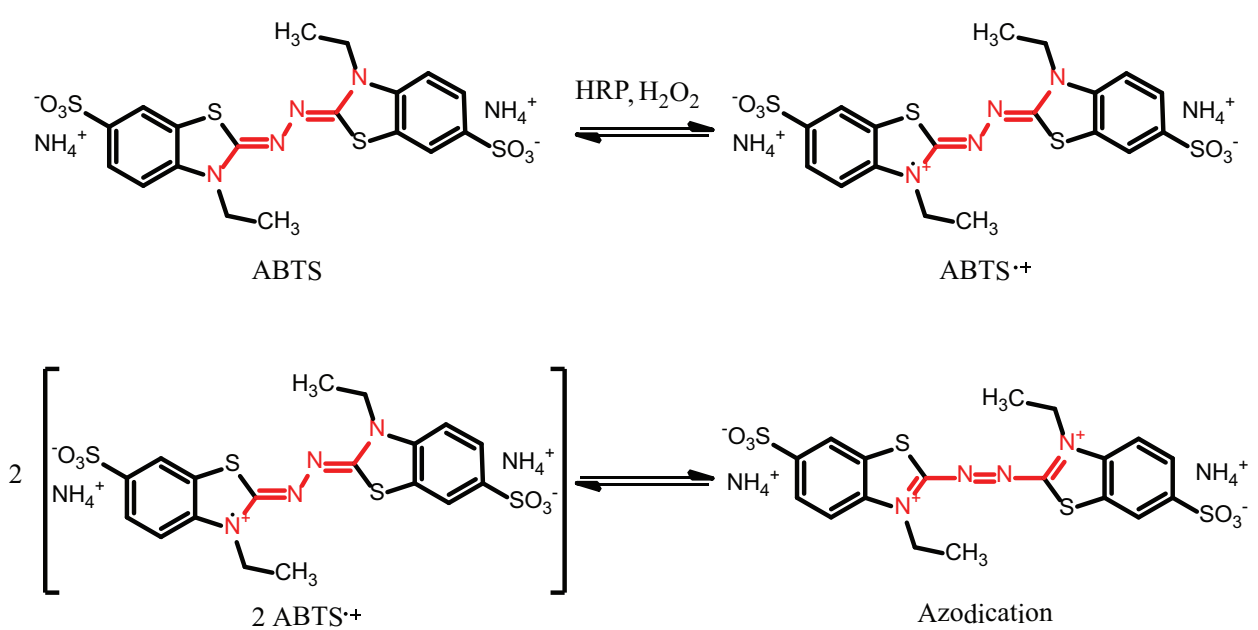

\subsubsection{Assay}

Evaluation of protein stability can be performed by monitoring the catalytic activity of the enzyme with a chromogen. The enzyme-substrate pair chosen for this work was horseradish peroxidase and hydrogen peroxide to oxidize the substrate 2,2'azino-di(3ethylbenzothiazoline-6-sulphonic acid) (ABTS) into a radical cation chromogenic product (Figure 5) that can be assessed on paper through imaging techniques. ${ }^{47,48,49}$ The reverse reduction reaction, as well as a disproportiation reaction to form an azodication, are in equilibrium with the radical cation. The assay must therefore be performed and the color intensity recorded in a time sensitive fashion.

\subsubsection{Shelf Life}

Enzyme stability and microPAD shelf life were evaluated by immobilizing horseradish peroxidase on paper under ambient laboratory conditions $\left(20^{\circ} \mathrm{C}, 30\right.$ to $60 \%$ relative humidity). The microPADs were be evaluated over a period of days, assessing enzyme activity. 
2. Methods and Materials

\subsection{Materials}

All materials were purchased or provided by commercial sources, unless otherwise stated. EPON ${ }^{\mathrm{TM}}$ Resin SU-8 (SU-8, Momentive) contained formaldehyde and polymer with 4,4' (1-methylethylidene)bis[phenol] and (chloromethyl)oxirane was pulverized, dissolved in acetone, and mixed to make a stock solution of $40 \%(w / w)$ SU-8 in acetone. EPONTM Resin SU-2.5 (SU-2.5, Momentive) contained formaldehyde, 4,4'-isopropylidenediphenolepichlorohydrin copolymer, and polymer with 4,4'-(1-methylethylidene)bis[phenol] and (chloromethyl)oxirane was dissolved in acetone, and mixed to make a stock solution of $40 \%$ (w/w) SU-2.5 in acetone. Triarylsulfonium hexafluorophosphate salts, mixed (initiator, Sigma-Aldrich) were used as received. A phosphate buffered saline, 10X solution, (PBS, Fisher Scientific) was diluted to a $1 \mathrm{X}$ solution with deionized water $\left(\mathrm{DI} \mathrm{H}_{2} \mathrm{O}\right)$. $\mathrm{D}(+)$-trehalose dihydrate (trehalose, Acros Organics), reagent grade, was prepared into a $1 \mathrm{M}$ (3.7846 g, 0.01 moles) solution in 1X PBS buffer. A 1-Step ${ }^{\mathrm{TM}}$ ABTS solution (ABTS, Thermo Scientific), containing proprietary concentrations hydrogen peroxide and 2,2'-azino-bis(3-ethylbenzothiazoline-6-sulphonic acid) diammonium salt, was used as received. Lyophilized peroxidase from horseradish (HRP, MP Biomedicals) was used as received. Polystyrene (PS, Polysciences) molecular weight 800 to 5000 and PS (Aldrich) molecular weight 30,000 were used as received. Whatman ${ }^{\circledR} \# 1$ chromatography paper (paper) and Whatman ${ }^{\circledR}$ Protran BA 85 Nitrocellulose (NC) were used as received.

\subsection{Microfluidic Paper-Based Analytical Device Design, Processing, and Development}

Before experimentation began, a microfluidic paper-based analytical device (microPAD) design was chosen to expedite the development and analysis of the survival of enzymes on a cellulose matrix. The simplest design incorporates a singular well - surrounded 
by a hydrophobic barrier, without channels - where the enzyme can be deposited until a substrate is added to perform an assay. The analogous platform within biology and biochemistry is the 96-well plate. The production of a 96-well "paper plate" (hereafter, referred to as microPAD or plate) and subsequent treatment of the microPADs are described below. Processing conditions described below reflect the final optimized steps for microPAD production, an in depth discussion of the evolution of the processing conditions can be found on page 33; unless explicitly stated the procedures detailed below, or a variant of the procedure, was used.

\subsubsection{Electronic Processing and Physical Creation}

A pattern corresponding to the dimensions seen in Figure 2.2.1 was produced in the software package CleWin version 2.89. This pattern was applied to Whatman® \#1 chromatography paper via a method known as wax printing. ${ }^{36}$ First, the pattern was printed on the paper using a Xerox Phaser 8560 printer. ${ }^{13}$ After printing, the wax was melted through the paper by heating the paper for 2 minutes within a MTI Corporation Compact Forced Air Convection Oven set to $195{ }^{\circ} \mathrm{C}$. Two microPADs were patterned on each page of $20 \mathrm{x}$ $20 \mathrm{~cm}^{2}$ chromatography paper. The plates were then cut to the final dimensions of the plate in preparation for microPAD processing. Additional designs, including the device pictured in Figure 2.2.1 were produced in the same manner as described above.

\subsubsection{Epoxy Novolac Resin Preparation}

An epoxy novolac resin was chosen to be impregnated within the cellulose matrix. The entirety of the plate was impregnated with an epoxy oligomer ranging in functionality from 2.5 to 8 average epoxy moieties. This was achieved through a mixture of EPONTM Resin SU-2.5 and EPONTM Resin SU-8 to achieve the desired functionality (hereafter, solutions containing either or both EPON" ${ }^{\mathrm{TM}}$ resins will be referred to as "SU-X" where "X" designates the calculated epoxide functionality - whether explicitly defined or otherwise). 
Using the appropriate stock solutions, as described in Section 2.1, solutions ranging from $0.625 \%(\mathrm{w} / \mathrm{w})$ to $30 \%(\mathrm{w} / \mathrm{w})$ were made by serial dilution with acetone. The corresponding mass of initiator (see Table 2.2.1) was then pipetted into solution SU-X and mixed. After mixing the initiator into the solution, the prepared photoresist was used (as described in Section 2.2.3) within 12 hours. See Table 2.2.1 for individual solution preparations.

Table 2.2.1.: Theoretical values for SU-X solution preparations.

\begin{tabular}{|c|c|c|c|c|}
\hline \multirow[b]{2}{*}{ Label } & \multicolumn{3}{|c|}{$\begin{array}{c}\text { Stock Solution Preparation } \\
\text { for } 100 \mathrm{~g}\end{array}$} & \multirow{2}{*}{$\begin{array}{c}\text { Mass per } 10 \mathrm{~g} \text { Sample } \\
\text { of Stock Solution Label } \\
\text { Theoretical } \\
\text { Mass Initiator }\end{array}$} \\
\hline & $\begin{array}{c}\text { Theoretical } \\
\%(w / w) \text { SU-X }\end{array}$ & $\begin{array}{l}\text { Theoretical } \\
\text { Mass SU-X }\end{array}$ & $\begin{array}{c}\text { Theoretical } \\
\text { Mass Acetone }\end{array}$ & \\
\hline A1 & $40 . \%$ SU-8 & 40 g SU-8 & $60 \mathrm{~g}$ & $0.250 \mathrm{~g}$ \\
\hline A2 & $30 . \%$ SU-8 & 40 g A1 & $60 \mathrm{~g}$ & $0.188 \mathrm{~g}$ \\
\hline A3 & $20 . \%$ SU-8 & $40 \mathrm{~g} \mathrm{~A} 2$ & $60 \mathrm{~g}$ & $0.125 \mathrm{~g}$ \\
\hline A4 & $10 . \%$ SU-8 & $40 \mathrm{~g} \mathrm{~A} 3$ & $60 \mathrm{~g}$ & $0.063 \mathrm{~g}$ \\
\hline A5 & $5.0 \%$ SU-8 & $40 \mathrm{~g} \mathrm{~A} 4$ & $60 \mathrm{~g}$ & $0.031 \mathrm{~g}$ \\
\hline A6 & $2.5 \% \mathrm{SU}-8$ & $40 \mathrm{~g}$ A5 & $60 \mathrm{~g}$ & $0.016 \mathrm{~g}$ \\
\hline A7 & $1.3 \% \mathrm{SU}-8$ & $40 \mathrm{~g}$ A6 & $60 \mathrm{~g}$ & $0.008 \mathrm{~g}$ \\
\hline A8 & $0.63 \% \mathrm{SU}-8$ & 40 g A7 & $60 \mathrm{~g}$ & $0.004 \mathrm{~g}$ \\
\hline B1 & $40 . \%$ SU-4 & $\begin{array}{l}10.8 \text { g SU-8, } \\
29.2 \text { g SU-2.5 }\end{array}$ & $60 \mathrm{~g}$ & $0.177 \mathrm{~g}$ \\
\hline B2 & $30 . \%$ SU-4 & 40 g B1 & $60 \mathrm{~g}$ & $0.133 \mathrm{~g}$ \\
\hline B3 & $20 . \%$ SU-4 & $40 \mathrm{~g} \mathrm{~B} 2$ & $60 \mathrm{~g}$ & $0.088 \mathrm{~g}$ \\
\hline B4 & $10 . \% \mathrm{SU}-4$ & $40 \mathrm{~g} \mathrm{~B} 3$ & $60 \mathrm{~g}$ & $0.044 \mathrm{~g}$ \\
\hline B5 & $5.0 \% \mathrm{SU}-4$ & $40 \mathrm{~g} \mathrm{~B} 4$ & $60 \mathrm{~g}$ & $0.022 \mathrm{~g}$ \\
\hline B6 & $2.5 \% \mathrm{SU}-4$ & $40 \mathrm{~g} \mathrm{~B} 5$ & $60 \mathrm{~g}$ & $0.011 \mathrm{~g}$ \\
\hline B7 & $1.3 \%$ SU-4 & $40 \mathrm{~g} \mathrm{~B} 6$ & $60 \mathrm{~g}$ & $0.005 \mathrm{~g}$ \\
\hline
\end{tabular}

\subsubsection{Photolithographic Process}

The unprocessed microPAD created by the process described in Section 2.2.1 was placed in a Pyrex dish. The SU-X solution prepared in Section 2.2.2 was distributed across the surface of the paper and allowed to completely impregnate the paper (Figure 2.2.2). Typically, this took only a seconds. Excess SU-X was removed from the the impregnated microPAD by dragging both the top and bottom of the paper across the edge of the Pyrex dish (Figure 2.2.3). The impregnated microPAD was then immediately placed within "the 


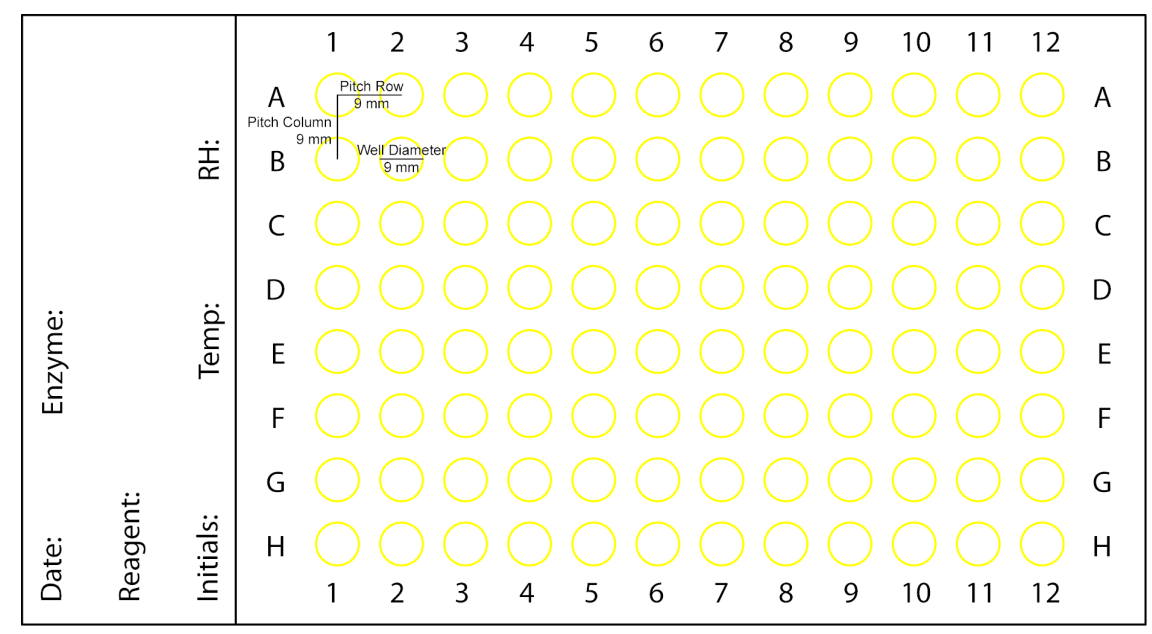

Figure 2.2.1.: A 96-well plate designed for wax patterning, using the same dimensions as a 96 -well plate.

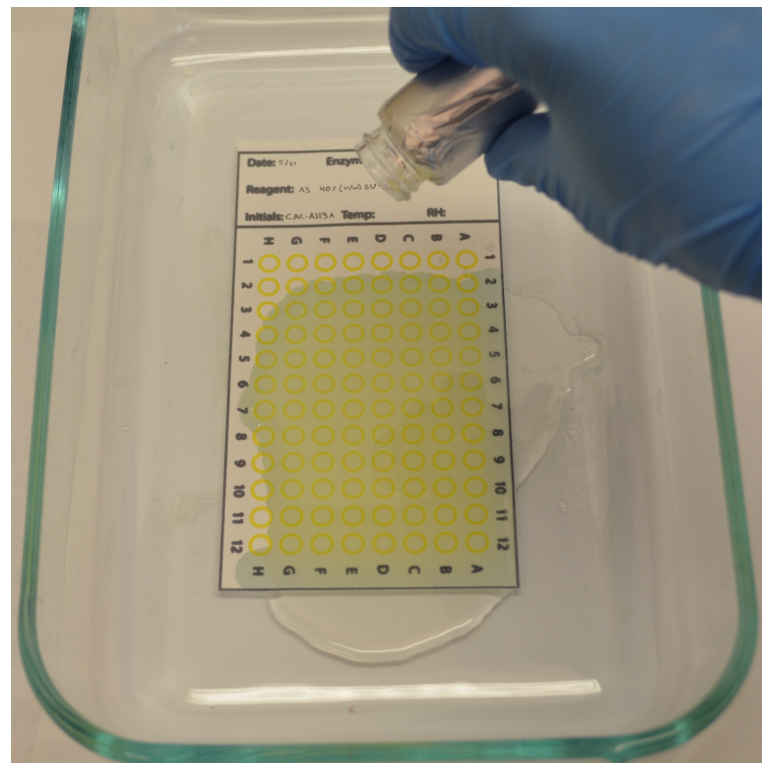

Figure 2.2.2.: A microPAD impregnated with $40 \%$ (w/w) SU-8 solution in acetone. 
box" in preparation for exposure, to minimize solvent evaporation (Figure 2.2.4). "The box" was an enclosure designed to hold the microPAD and UV source in a controlled environment.

The microPAD was exposed for 20 minutes under long wave ultraviolet light $(365 \mathrm{~nm})$ with a Spectroline Model ENF-240C (300 $\mu \mathrm{W} / \mathrm{cm}^{2}$ or output ). The exposed microPAD then underwent both a post-bake and hard bake at $65^{\circ} \mathrm{C}$ and $95{ }^{\circ} \mathrm{C}$, respectively, for 10 minutes each, on a Cimarec ${ }^{\mathrm{TM}}$ ceramic top hot plate (model HP131225). The microPAD was then developed by soaking it in an acetone bath for approximately one hour with slight agitation, followed by a rinse in IPA. The development steps were repeated until no white residues were observed. The microPAD was then complete and ready to be used with reagents.

\subsubsection{Alternative Polymer Matrices}

Alternate polymer matrices explored include: polystyrene (PS) 800 to 5000 molecular weight, nitrocellulose (NC), SU-8 with $30 \mathrm{~K}$ molecular weight PS, and SU-8 with 800 to 5000 molecular weight PS, all prepared as weight percents in acetone (see Table 2.2.2 on page 26). PS and NC solutions were applied similarly to the process described in Section 2.2.3. Half of the prepared microPADs were primed for reagent delivery. This priming consisted of soaking a microPAD for 30 minutes in a $\mathrm{DI} \mathrm{H}_{2} \mathrm{O}$ bath, or until the water had completely penetrated the microPAD. The primed microPADs were air dried overnight, after which reagents were ready to be added. SU-8 solutions containing PS were applied to the microPADs as described in Section 2.2.3. 


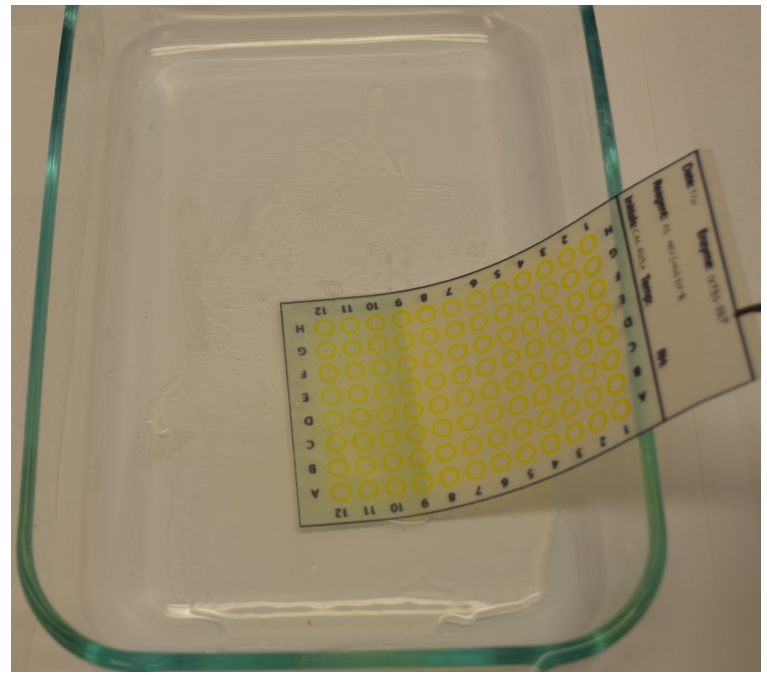

Figure 2.2.3.: Removing excess solution from microPAD
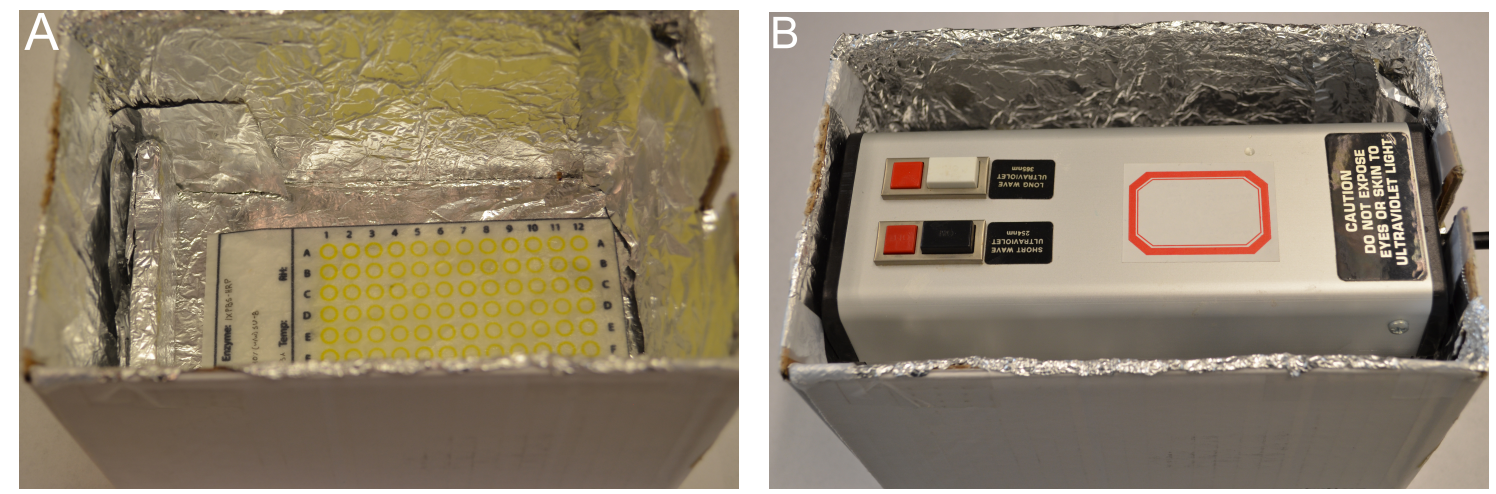

Figure 2.2.4.: "The box" is used as an exposure chamber for SU-X impregnated microPADs: (a) "The box" with a microPAD awaiting exposure. The UV lamp sits completely within the box, and is elevated $4 \mathrm{~cm}$ above the floor of "the box:" (b) the UV lamp in place within "the box," ready for the exposure. 


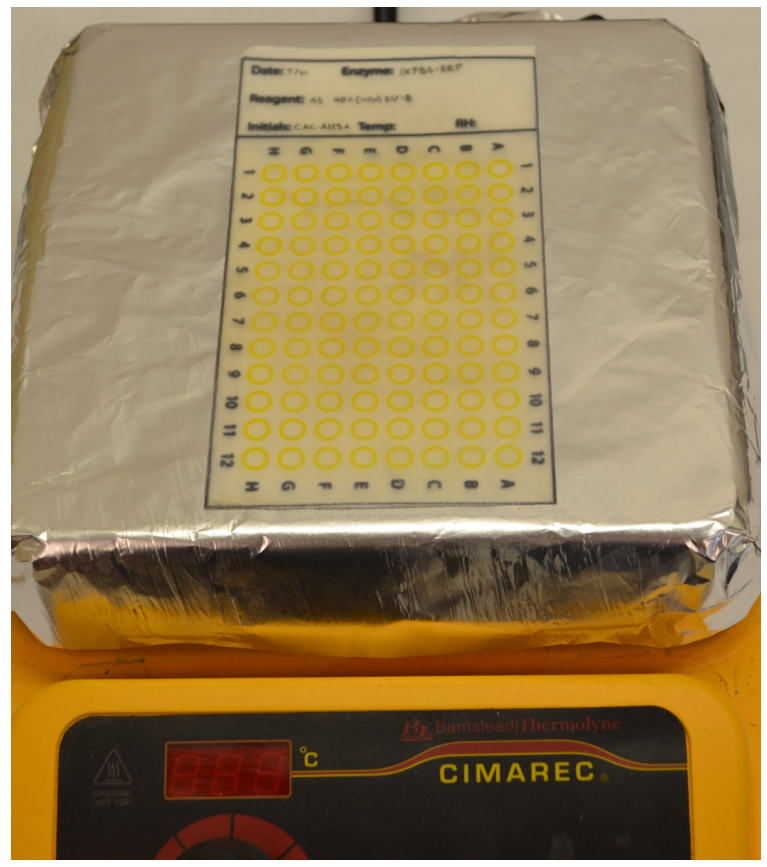

Figure 2.2.5.: Post and hard bake setup for microPAD.
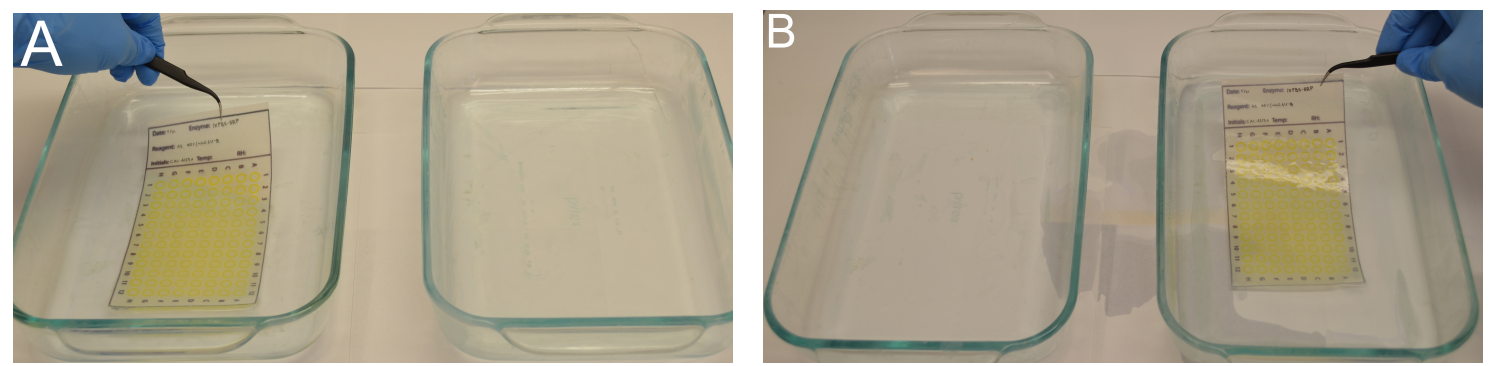

Figure 2.2.6.: MicroPAD development: (a) acetone bath, and (b) IPA rinse. 
Table 2.2.2.: Alternate Polymer Matrices Solution Preparation.

\begin{tabular}{|c|c|c|c|c|}
\hline \multirow[b]{2}{*}{ Label } & \multicolumn{4}{|c|}{ Solution Preparation } \\
\hline & $\begin{array}{c}\text { Theoretical } \\
\%(w / w) \text { Polymer }\end{array}$ & $\begin{array}{l}\text { Theoretical } \\
\text { Mass Polymer }\end{array}$ & $\begin{array}{l}\text { Theoretical } \\
\text { Mass Acetone }\end{array}$ & $\begin{array}{l}\text { Theoretical } \\
\text { Mass Initiator }\end{array}$ \\
\hline A6-1 & $2.5 \% \mathrm{SU}-8$ & $0.470 \mathrm{~g} \mathrm{~A} 6$ & $17.590 \mathrm{~g}$ & $0.016 \mathrm{~g}$ \\
\hline A6-2 & $2.5 \%$ SU-8 & $0.470 \mathrm{~g} \mathrm{~A} 6$ & $17.590 \mathrm{~g}$ & $0.016 \mathrm{~g}$ \\
\hline $\mathrm{NC} 1$ & $2.5 \% \mathrm{NC}$ & $0.522 \mathrm{~g}$ & $17.596 \mathrm{~g}$ & $x$ \\
\hline $\mathrm{NC} 2$ & $2.5 \% \mathrm{NC}$ & $0.522 \mathrm{~g}$ & $17.596 \mathrm{~g}$ & $x$ \\
\hline PS1 & $\begin{array}{c}2.5 \% \text { PS } \\
800-5000 \mathrm{MW}\end{array}$ & $0.25 \mathrm{~g}$ PS & $9.75 \mathrm{~g}$ & $x$ \\
\hline PS2 & $\begin{array}{c}2.5 \% \text { PS } \\
800-5000 \mathrm{MW}\end{array}$ & $0.25 \mathrm{~g}$ PS & $9.75 \mathrm{~g}$ & $x$ \\
\hline PS3 & $\begin{array}{c}4 \% \text { PS } \\
800-5000 \mathrm{MW}\end{array}$ & $\begin{array}{c}15 \mathrm{~g} \mathrm{~A} 1, \\
0.586 \mathrm{~g} \text { PS }\end{array}$ & $x$ & $x$ \\
\hline PS4 & $4 \%$ PS $30 \mathrm{~K} \mathrm{MW}$ & $\begin{array}{c}15 \mathrm{~g} \mathrm{~A} 1, \\
0.586 \mathrm{~g} \mathrm{PS}\end{array}$ & $x$ & $x$ \\
\hline
\end{tabular}




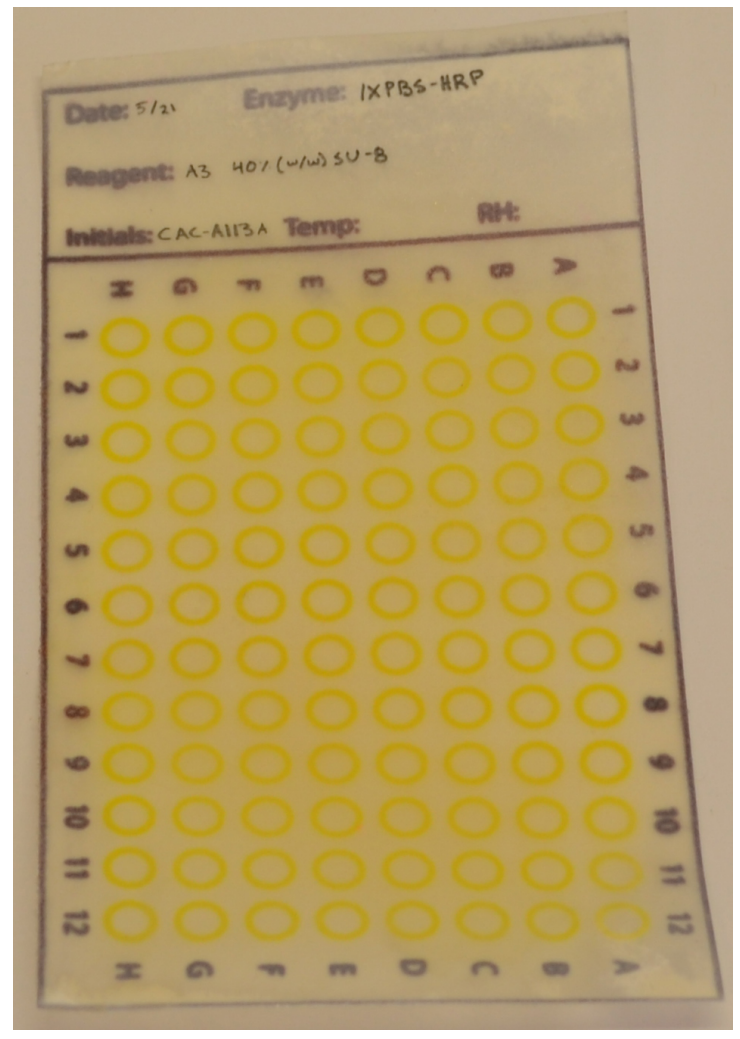

Figure 2.2.7.: Completed microPAD 96-well paper plate. 


\subsection{Microfluidic Paper-Based Analytical Device Characterization}

Several attempts were made to characterize the success of SU-X polymerizations within a cellulose matrix, as well as the total SU-X delivered to the microPAD, and the total SU-X polymerized in the paper. The efforts made are described below, their success and potential results are discussed in Section 3.2.

\subsubsection{Fourier Transform Infrared Spectroscopy}

Fourier transform infrared (FTIR) absorbance spectra were obtained on a Nicolet iS10 FT-IR spectrometer with a DTGS KBr detector (Thermo Scientific) in attenuated total reflection mode at a resolution of $0.5 \mathrm{~cm}^{-1}$ for 512 scans. Samples analyzed by FTIR spectroscopy include paper, paper after microPAD fabrication without photoresist, and microPADs processed with Solution A1 (Table 2.2.1) after impregnation (pre-exposure), after exposure, and after the post-exposure/hard bake. MicroPADs processed with solutions A2 through A8 were analyzed post-hard bake.

\subsubsection{Microfluidic Paper-Based Analytical Device Hydrophobicity}

After processing, an aliquot of $3 \mu \mathrm{L}$ deionized $\mathrm{H}_{2} \mathrm{O}$ was placed on the surface of the sample with a micropipette. The potential wicking ability of the microPAD was evaluated by whether the water was immediately absorbed into the microPAD, absorbed when left on the surface for less than 5 minutes, absorbed into the microPAD in more than 5 minutes, or failed to absorb into the microPAD.

Qualitative testing was performed in order to determine the physical processing conditions required to allow fully processed microPADs to accept reagents into the cellulose/SU$\mathrm{X}$ matrix. Paper squares $\left(2.5 \times 2.5 \mathrm{~cm}^{2}\right)$ were cut out and processed in several ways:

1. As in the photolithographic process described in Section 2.2.3.

2. Without exposure, both with and without acetone development. 
3. Variable pre-bake, post-bake, and hard-bake time and temperatures.

4. Variable acetone, IPA wash times.

\subsection{Horseradish Peroxidase Assay}

As discussed in Section 1.3.3, the enzyme HRP was chosen to perform a colorimetric assay with the substrate ABTS. HRP was chosen for this assay because of its extensive use in biochemistry when coupled with other reactions to amplify a weak signal, ${ }^{47}$ as well as its use in clinically relevant diagnostic tests. ${ }^{49}$ ABTS was chosen for this assay due to its common use with peroxidases. In addition, the product of the HRP-ABTS assay will result in an even distribution of colored product in the test zone and does not exhibit a 'coffee-ring' effect. ${ }^{39}$

\subsubsection{Assay Solution Preparation}

A 1X PBS solution was used throughout assay preparation. A $1 \mathrm{~mL}$ stock solution of HRP (40.4 mg) was prepared in 1X PBS, from which all HRP dilutions were made. The enzyme stock solution, and all other solutions containing enzyme were stored in a freezer at $-20^{\circ} \mathrm{C}$. Dilutions were prepared in $1 \mathrm{X}$ PBS to a dilution factor (DF) of 32 . A 32 DF HRP solution was prepared in IPA (IPA-HRP) immediately prior to use. Precipitates were allowed to settle out of the IPA-HRP solution, and the supernatant was decanted to a storage container. A substrate solution was prepared in IPA at a 50\% (v/v) with the ABTS solution (IPA-ABTS). The ABTS solution and $1 \mathrm{M}$ trehalose solution were used and prepared respectively as was described in Section 2.1.

\subsubsection{Microfluidic Paper-Based Analytical Device Implementation (Shelf Life)}

Table 2.4.1 on the next page outlines the conditions of each different assay performed on the microPADs for shelf life experiments. All concentrations are given as weight percent. Reagents in water, buffer, or IPA were added to microPAD wells through the use of a 
Table 2.4.1.: Individual microPAD assay conditions, " $\sqrt{\mathrm{s}}$ " indicating reagents used.

\begin{tabular}{|c|c|c|c|c|c|c|c|}
\hline Label & Solution Delivered & 1-M Trehalose & HRP & IPA-HRP & ABTS & IPA-ABTS & $\begin{array}{c}\text { Storage: } \\
21^{\circ} \mathrm{C} 4{ }^{\circ} \mathrm{C}\end{array}$ \\
\hline SSE-XF & $x$ & $x$ & $\sqrt{ }$ & $x$ & $x$ & $\sqrt{ }$ & $4^{\circ} \mathrm{C}$ \\
\hline SSE-IF & $x$ & $x$ & $x$ & $\sqrt{ }$ & $x$ & $\sqrt{ }$ & $4^{\circ} \mathrm{C}$ \\
\hline SSE-XR & $x$ & $x$ & $\sqrt{ }$ & $x$ & $x$ & $\sqrt{ }$ & $21{ }^{\circ} \mathrm{C}$ \\
\hline SSE-IR & $x$ & $x$ & $x$ & $\sqrt{ }$ & $x$ & $\sqrt{ }$ & $21{ }^{\circ} \mathrm{C}$ \\
\hline $\mathrm{C} 1$ & $x$ & $x$ & $\sqrt{ }$ & $x$ & $\sqrt{ }$ & $x$ & $21^{\circ} \mathrm{C}$ \\
\hline $\mathrm{C} 2$ & $x$ & $\checkmark$ & $\sqrt{ }$ & $x$ & $\sqrt{ }$ & $x$ & $21{ }^{\circ} \mathrm{C}$ \\
\hline A5-1 & $5.0 \%$ SU-8 (A5) & $\sqrt{ }$ & $\checkmark$ & $x$ & $\sqrt{ }$ & $x$ & $21^{\circ} \mathrm{C}$ \\
\hline A6-3 & $2.5 \%$ SU-8 (A6) & $\sqrt{ }$ & $\sqrt{ }$ & $x$ & $\sqrt{ }$ & $x$ & $21^{\circ} \mathrm{C}$ \\
\hline A7-1 & $1.3 \%$ SU-8 (A7) & $\checkmark$ & $\checkmark$ & $x$ & $\sqrt{ }$ & $x$ & $21{ }^{\circ} \mathrm{C}$ \\
\hline A8-1 & $0.63 \%$ SU-8 (A8) & $\sqrt{ }$ & $\sqrt{ }$ & $x$ & $\sqrt{ }$ & $x$ & $21^{\circ} \mathrm{C}$ \\
\hline A5-2 & $5.0 \%$ SU-8 (A5) & $x$ & $\checkmark$ & $x$ & $\sqrt{ }$ & $x$ & $21^{\circ} \mathrm{C}$ \\
\hline A6-4 & $2.5 \%$ SU-8 (A6) & $x$ & $\checkmark$ & $x$ & $\checkmark$ & $x$ & $21{ }^{\circ} \mathrm{C}$ \\
\hline A7-2 & $1.3 \%$ SU-8 (A7) & $x$ & $\sqrt{ }$ & $x$ & $\sqrt{ }$ & $x$ & $21^{\circ} \mathrm{C}$ \\
\hline A8-2 & $0.63 \%$ SU-8 (A8) & $x$ & $\sqrt{ }$ & $x$ & $\sqrt{ }$ & $x$ & $21^{\circ} \mathrm{C}$ \\
\hline A6-5 & $2.5 \%$ SU-8 (A6) & $x$ & $\sqrt{ }$ & $x$ & $\sqrt{ }$ & $x$ & $21^{\circ} \mathrm{C}$ \\
\hline A6-6 & $2.5 \mathrm{SU}-8$ (A6) & $x$ & $\checkmark$ & $x$ & $\sqrt{ }$ & $x$ & $21^{\circ} \mathrm{C}$ \\
\hline $\mathrm{NC1}$ & $2.5 \% \mathrm{NC}(\mathrm{N} 1)$ & $x$ & $\sqrt{ }$ & $x$ & $\sqrt{ }$ & $x$ & $21^{\circ} \mathrm{C}$ \\
\hline $\mathrm{NC} 2$ & $2.5 \% \mathrm{NC}(\mathrm{N} 1)$ & $x$ & $\checkmark$ & $x$ & $\sqrt{ }$ & $x$ & $21^{\circ} \mathrm{C}$ \\
\hline PS1 & $2.5 \%$ PS (P1) & $x$ & $\sqrt{ }$ & $x$ & $\sqrt{ }$ & $x$ & $21^{\circ} \mathrm{C}$ \\
\hline PS2 & $2.5 \%$ PS (P1) & $x$ & $\checkmark$ & $x$ & $\sqrt{ }$ & $x$ & $21^{\circ} \mathrm{C}$ \\
\hline A2-1 & $30 \%$ SU-8 (A2) & $x$ & $x$ & $\sqrt{ }$ & $x$ & $\sqrt{ }$ & $21^{\circ} \mathrm{C}$ \\
\hline A3-1 & $20 \%$ SU-8 (A3) & $x$ & $x$ & $\sqrt{ }$ & $x$ & $\sqrt{ }$ & $21^{\circ} \mathrm{C}$ \\
\hline A4-1 & $10 \%$ SU-8 (A4) & $x$ & $x$ & $\checkmark$ & $x$ & $\sqrt{ }$ & $21^{\circ} \mathrm{C}$ \\
\hline B2-1 & $30 \%$ SU-4 (B2) & $x$ & $x$ & $\sqrt{ }$ & $x$ & $\checkmark$ & $21^{\circ} \mathrm{C}$ \\
\hline B3-1 & $20 \%$ SU-4 (B3) & $x$ & $x$ & $\sqrt{ }$ & $x$ & $\sqrt{ }$ & $21^{\circ} \mathrm{C}$ \\
\hline B4-1 & $10 \%$ SU-4 (B4) & $x$ & $x$ & $\sqrt{ }$ & $x$ & $\sqrt{ }$ & $21^{\circ} \mathrm{C}$ \\
\hline A7-3 & $1.3 \%$ SU-8 (A7) & $x$ & $x$ & $\checkmark$ & $x$ & $\checkmark$ & $21^{\circ} \mathrm{C}$ \\
\hline A7-4 & $1.3 \%$ SU-8 (A7) & $x$ & $\sqrt{ }$ & $x$ & $x$ & $\sqrt{ }$ & $21^{\circ} \mathrm{C}$ \\
\hline A6-7 & $2.5 \%$ SU-8 (A6) & $x$ & $x$ & $\checkmark$ & $x$ & $\checkmark$ & $21^{\circ} \mathrm{C}$ \\
\hline A6-8 & $2.5 \%$ SU-8 (A6) & $x$ & $\sqrt{ }$ & $x$ & $x$ & $\sqrt{ }$ & $21^{\circ} \mathrm{C}$ \\
\hline A1-1 & $40 \%$ SU-8 (A1) & $x$ & $x$ & $\sqrt{ }$ & $x$ & $\sqrt{ }$ & $21^{\circ} \mathrm{C}$ \\
\hline A1-2 & $40 \%$ SU-8 (A1) & $x$ & $\sqrt{ }$ & $x$ & $x$ & $\sqrt{ }$ & $21^{\circ} \mathrm{C}$ \\
\hline A4-2 & $10 \%$ SU-8 (A4) & $x$ & $\checkmark$ & $x$ & $x$ & $\sqrt{ }$ & $21^{\circ} \mathrm{C}$ \\
\hline B1-1 & $40 \%$ SU-4 (B1) & $x$ & $x$ & $\sqrt{ }$ & $x$ & $\sqrt{ }$ & $21^{\circ} \mathrm{C}$ \\
\hline B1-2 & $40 \%$ SU-4 (B1) & $x$ & $\sqrt{ }$ & $x$ & $x$ & $\checkmark$ & $21^{\circ} \mathrm{C}$ \\
\hline
\end{tabular}


Formulatrix Mantis Liquid Dispenser. Reagents containing acetone were manually pipetted into each well with an Eppendorf Research Plus micropipette. Given below are the reagent order of addition, reagent volume, and reagent concentration, respectively, of each reagent added to a microPAD test zone.

1. $1 \mu \mathrm{L}$, polymer in acetone (A5-1, A6-3, A7-1, A8-1, A5-2, A6-4, A7-2, A8-2, A6-5, A6-6, NC-1, NC-2, PS-1, PS-2); if label is not given the entirety of the microPAD was coated with polymer.

2. $1 \mu \mathrm{L}, 1 \mathrm{M}$ trehalose (C2, A5-1, A6-3, A7-1, A8-1).

3. $1 \mu \mathrm{L}, \mathrm{HRP}$ (SSE-XF, SSE-XR, C1, C2, A5-1, A6-3, A7-1, A8-1, A5-2, A6-4, A7-2, A8-2, A6-5, A6-6, NC-1, NC-2, PS-1, PS-2) or $1 \mu \mathrm{L}$, IPA-HRP (SSE-IF, SSE-IR, A2-1, A3-1, A4-1, B2-1, B3-1, B4-1, A7-3, A6-7, A1-1, B1-1).

4. $3 \mu \mathrm{L}$, ABTS (A5-1, A6-3, A7-1, A8-1, A5-2, A6-4, A7-2, A8-2, A6-5, A6-6, NC1, NC-2, PS-1, PS-2) or two $3 \mu \mathrm{L}$ aliquots, IPA-ABTS (SSE-XF, SSE-XR, SSE-IF, SSE-IR, A2-1, A3-1, A4-1, B2-1, B3-1, B4-1, A7-3, A7-4, A6-7, A6-8, A1-1, A1-2, A4-2, B1-1, B1-2).

Steps one through three were performed following the completion of the photolithographic process. Step four is time sensitive, and was performed on the initial day as soon as possible after step three was completed. Step four was then repeated on subsequent days based on the degradation of signal from the enzyme loaded microPAD. All microPADs were stored at room temperature under ambient laboratory conditions.

Additional control experiments were run to determine the activity of HRP in solution (SSE, for 1X PBS and IPA) when stored at both room temperature and $4{ }^{\circ} \mathrm{C}$, the activity of HRP with (C1, no stabilizing agents) on paper, and the activity of HRP stabilized with dried trehalose $(\mathrm{C} 2)$ on paper. These experiments utilized steps one through four in the same manner as described above. 


\subsubsection{Microfluidic Paper-Based Analytical Device Post-Assay Processing}

Thirty minutes after the addition of the substrate (ABTS or IPA-ABTS) to all designated wells, the microPAD was imaged with an Epson Perfection V300 photo scanner at 48-bit color, 300 dpi to the least compressed image quality available for a JPEG. The software package ImageJ version 1.46r was then used to process each image. Within ImageJ, the image was manipulated by inverting the image (producing its negative), splitting the image into RBG colors and analyzing the red channel. This was done in order to minimize the signal from yellow wax wells. Using the produced image, the color intensity of each assay's well was recorded. Microsoft Excel was then used for data processing. 


\section{Results and Discussion}

\subsection{Microfluidic Paper-Based Analytical Device Design, Processing, and Development}

\subsubsection{Photolithographic Process}

The photolithographic process is typically split into six distinct parts: photoresist application, pre-bake, exposure, post-exposure bake, hard bake, and development. Photolithography on paper utilizes all of these steps to some extent, although their importance is less defined when considering the processes described within this manuscript. Here the microPADs lack defined structures which typically characterize SU-8 applications; instead, the microPADs will ideally be characterized by uniform photoresist thickness and crosslink density. Therefore, many of the conditions of the photolithographic process were varied in order to determine the ideal conditions to maximize throughput of the microPADs and maximize their utility of the resulting devices for applications in diagnostics.

The process described in Section 2.2.3 is not truly optimized, it merely represents the accumulation of many tests performed to obtain a consistent microPAD.

\subsubsection{Photoresist application}

Unlike a microelectromechanical system (MEMS) application for photoresists, spincoating was not a practical method for applying photoresist to paper. Consider that instead of forming a film of photoresist on top of a substrate, such as a silicon wafer, the photoresist was impregnated (absorbed) into the cellulose matrix of the paper. Ideally, excess photoresist would not be present to form a film outside the thickness of the paper. However, this cannot be ensured due to the current processing techniques used.

The first issue arose with incomplete impregnation of photoresist into the microPAD. This was characterized by a fast evaporation of solvent, leaving behind a largely viscous portion of photoresist that did not visually penetrate to the back of the paper. Initial mi- 
croPADs were produced by pipetting SU-8 solution onto paper and ensuring impregnation with a wooden roller. This technique was discarded because the wooden roller did not assist the photoresist penetrating into the paper once the solvent had evaporated to a large extent. Instead, larger photoresist solution volumes were produced in order to completely saturate the paper. With these larger solution volumes, excess photoresist was consistently present at higher photoresist concentrations due to elevated solution viscosity. To remove this excess photoresist, the impregnated paper was dragged across the inside edge of a Pyrex dish. This had the effect of containing excess photoresist solution and removing to a visually acceptable degree excess solution on the top and bottom of the impregnated paper.

\subsubsection{Pre-Bake}

Pre-bake conditions were explored early on in the optimization process. The pre-bake (also known as a soft bake) is used in the photolithographic process to drive solvents from the polymer film, and dry the film for subsequent handling. ${ }^{27}$ It was hypothesized that by forcing the photoresist to undergo rapid thermal expansion, the permeability of the resulting film would allow for reagent addition with the resulting microPAD due to a large presence of microcracking within the polymer.

Holding all other photolithographic steps constant, a variety of pre-bake temperatures were explored using a $40 \%(\mathrm{w} / \mathrm{w}) \mathrm{SU}-8$ solution. This high concentration was used because successful cross-linking could be determined based on the appearance of the fully processed microPAD (e.g. Figure 2.2.7). The permeability of these microPADs were tested by dipping the edge of the processed microPAD in a solution of blue dye. It was found that over the range of $30^{\circ} \mathrm{C}$ to $150{ }^{\circ} \mathrm{C}$ baking temperatures, and one min to one hour baking times there is no large difference with the permeability of the microPADs. This result was largely obfuscated by the incomplete polymerization of SU-X, which could have been due to a lack of photoinitiation, low degree of crosslinking, or early termination of the polymer chains. Ultimately, the highest number of successful polymerizations were performed when 
the solvent evaporation between the photoresist application and exposure was minimized; therefore, no pre-bake was performed in the optimized process. For devices not processed with an exposure, a pre-bake at $65^{\circ} \mathrm{C}$ was performed for 20 minutes.

\subsubsection{Exposure}

As discussed in Section 1.3.1, exposure forms a strong acid from the triarylsulfonium salt initiator. The production of microPADs utilized two means of exposure: 1) sunlight, and 2) UV lamp. The effectiveness of different exposure types and exposure times was explored qualitatively to determine the most effective exposure to produce microPADs. Sunlight exposures were performed for a range of exposure times from five to thirty minutes on days when the US weather service reported a UV index of nine or greater. UV lamp exposures were performed both within "the box" and in an open environment for a range of exposure times ranging from five to twenty minutes.

Sunlight exposures produced variable results. It was seen that exposure times of less than 15 minutes, no matter the day the experiment was performed on, would not produce crosslinked SU-8 as determined in Section 3.1.1.2, with $40 \%$ (w/w) SU-8 impregnated paper. At exposure times greater than 15 minutes, the highest number of successful polymerizations was performed at 30 minutes. However, even with exposure times maximized at 30 minutes, exposures via sunlight succeeded only approximately 50 percent of the time. It is the conclusion of the author, that polymerizations via sunlight exposure succeeded only when: 1) enough solvent remained to enable the photoinitiation from the photoacid, and/or 2) the temperature of the polymerization platform (aluminum foil) was raised to a high enough temperature to drive the acid-catalyzed, thermally-driven crosslinking often seen in the post-exposure bake (Section 3.1.1.4). Controlling and testing the variables necessary to optimize sunlight exposures was outside the scope of this project, however novel the results may have been. Therefore, the use of a UV lamp was explored. 
The Spectroline UV lamp used was adapted for use with photoresist exposure processing. The exposure energy at the maximum exposure time of 20 minutes was $360 \mathrm{~mJ} / \mathrm{cm}^{2}$. This exposure is well in excess to the reported exposure dosages for the reported film thicknesses provided by Micro chem for SU-8 $2000\left(160 \mathrm{~mJ} / \mathrm{cm}^{2}\right.$ for a thickness of 40 microns) in the technical data sheet. ${ }^{26}$ Several issues arose when exposing impregnated microPADs with the UV lamp. The first was incomplete or uninitiated crosslinking of the impregnated microPAD which was apparent from a visible lack of color change in the impregnated microPAD. The next issue appeared only when exposure was performed in an open-environment (counter-top), where the SU-8 was selectively polymerized in the area directly below the UV lamp. This was expected, but undesired, because the UV lamp acts as its own UV mask by preventing line-of-sight from the lamp to the photoresist for portions of the microPAD. In order to fully expose the microPAD to the UV light, the lamp was first elevated from four centimeters to eight centimeters above the microPAD. It was eventually concluded that in doing so, the exposure dosage would have to be increased to an unreasonable extent. Instead, "the box" was produced to encourage total coverage with UV light to produce photoacid at the edges of the microPAD. The product of this process can be seen in Figure 2.2.7 on page 27.

\subsubsection{Post-Exposure and Hard Bakes}

Early experiments with sunlight exposure did not utilize the post-exposure bake (PEB) or hard bake, instead they relied on ambient conditions and photoinitiation providing the driving force for propagation of polymer chains and crosslinking. Initial experiments underestimated the importance of the PEB and hard bake. It was only after testing varying exposures settings during the open-environment UV lamp trials that it became apparent that thermally driving the polymerization at higher temperatures was necessary. The PEB and hard bake conditions combined with exposure conditions discussed in Section 2.2.3 yielded

successful polymerizations with each microPAD produced under the combined conditions. 


\subsubsection{Development}

The development process consisted of two steps. The first, a wash in developer, was meant to remove unreacted monomer within the film. The second, an IPA wash, terminates the cationic polymerization by chain transfer and precipitated monomer to form a white, hazy solution. For a typical photolithographic application, development is a key step in forming high resolution microstructures. For applications with microPADs, this is typically less important. However, removing excess monomer from the microPAD surface has the potential to reduce hydrophobicity of the final microPAD. More important is that development allows for the characterization and comparison of the effect of unreacted monomer on the enzyme shelf-life on a microPAD. This is discussed more in Sections 3.3.1 and 3.3.3.

\subsubsection{Alternative Polymer Matrices}

Physical modifications to paper with PS 800-5000 molecular weight and NC, compared to SU-8, was performed to determine the effect different polymer structures had on the shelf life of horseradish peroxidase. The modification of paper with these polymers was simple and effective, only requiring the polymer to be impregnated into the cellulose matrix. The results of the shelf-life experiments with these microPADs are given in Section 3.3.2.

Modification of the SU-8 film was also attempted with SU-8 with 30K molecular weight PS and SU-8 with 800 to 5000 molecular weight PS, similar to Walheim et al. where thin polymer films were produced with sacrificial polystyrene to increase the performance of antireflective coatings. ${ }^{50}$ Ideally, the films with incorporated PS could be bathed in acetone, removing the PS from the film. The increased porosity of the SU-8 film would then allow for the addition of reagents to the microPAD even at high concentrations of SU-X. This was not found to be the case. The resulting microPAD did not appear to allow for aqueous solutions to penetrate the SU-8. Visually, there appeared to be a increase in the wetting at the surface of the microPAD; however, at least at these lower concentrations of PS, the use 
of PS incorporated into SU-8 films proved inadequate for reagent addition to microPADs and was not pursued further.

\subsection{Microfluidic Paper-Based Analytical Device Characterization}

The characterization of the discussed microPADs was performed in order to better understand how variations in processing affect the shelf life of an enzyme on the microPAD. Fourier transform infrared spectroscopy, was performed in order to determine the success of the photoresist polymerization during the photolithographic process.

\subsubsection{FTIR}

Infrared spectrometers use two modes of emitting and gathering infrared electromagnetic radiation (EMR): transmission and reflection. Transmission spectroscopy requires pressing a solid sample between two inorganic salt discs. A non-volatile liquid sample can be prepared in much the same way; however, a volatile liquid must be placed in a sealed cell to prevent evaporation. This sample preparation is a clear drawback, but what may be even worse with this methodology is that the sample must be transparent for the analysis to be successful. Thus the number of samples which may be analyzed by this method is limited. Alternatively, attenuated total reflectance (ATR) spectroscopy can be used. Since now both the emission source and receiver are on the same side of the sample, only the optics of the machine must be transparent to transmit the EMR to the sample. The instrument used in this experiment employs a zinc selenide salt sandwiched between a glass substrate and the sample. Radiation is emitted from a source, enters the crystal, and is internally reflected many times before exiting the crystal. Each reflection will penetrate the sample up to a few micrometers, resulting in a selective attenuation of the radiation at the wavelengths the sample absorbs at. With this method many more samples may be analyzed because the sample is not limited to only transparent materials, but instead may include opaque materials. $^{51}$ 
ATR FTIR spectroscopy was used here to qualitatively determine the extent of crosslinking with SU-X systems by monitoring the loss of signal from the carbon-oxygen stretching of the cis or trans substituted epoxy rings in the monomer at 861 or $910 \mathrm{~cm}^{-1}$ respectively at the surface of the paper (a full study of the crosslinking as a function of the paper depth was not explored). ${ }^{52,27}$ Alternatively, the appearance of carbon-oxygen-carbon stretching in ethers can be monitored in the range of 1000 to $1230 \mathrm{~cm}^{-1} \cdot{ }^{27}$ However, ether stretches of the latter can overlap with carbon-oxygen-carbon ether stretching present in cellulose. Figure 3.2.1 shows the FTIR spectrum of cellulose, here the spectrum shows a very broad stretch across approximately 900 to $1200 \mathrm{~cm}^{-1}$ with a small, but distinguished stretch labeled at $898 \mathrm{~cm}^{-1}$. Figure 3.2.1 depicts paper which underwent only the development step of the processing (acetone and IPA washes), this spectrum is significant because it highlights that even after an hour of air drying, a carbon-oxygen stretch is present at 831 $\mathrm{cm}^{-1}$. The FTIR spectra of acetone and IPA are given in the Appendix A for comparison.

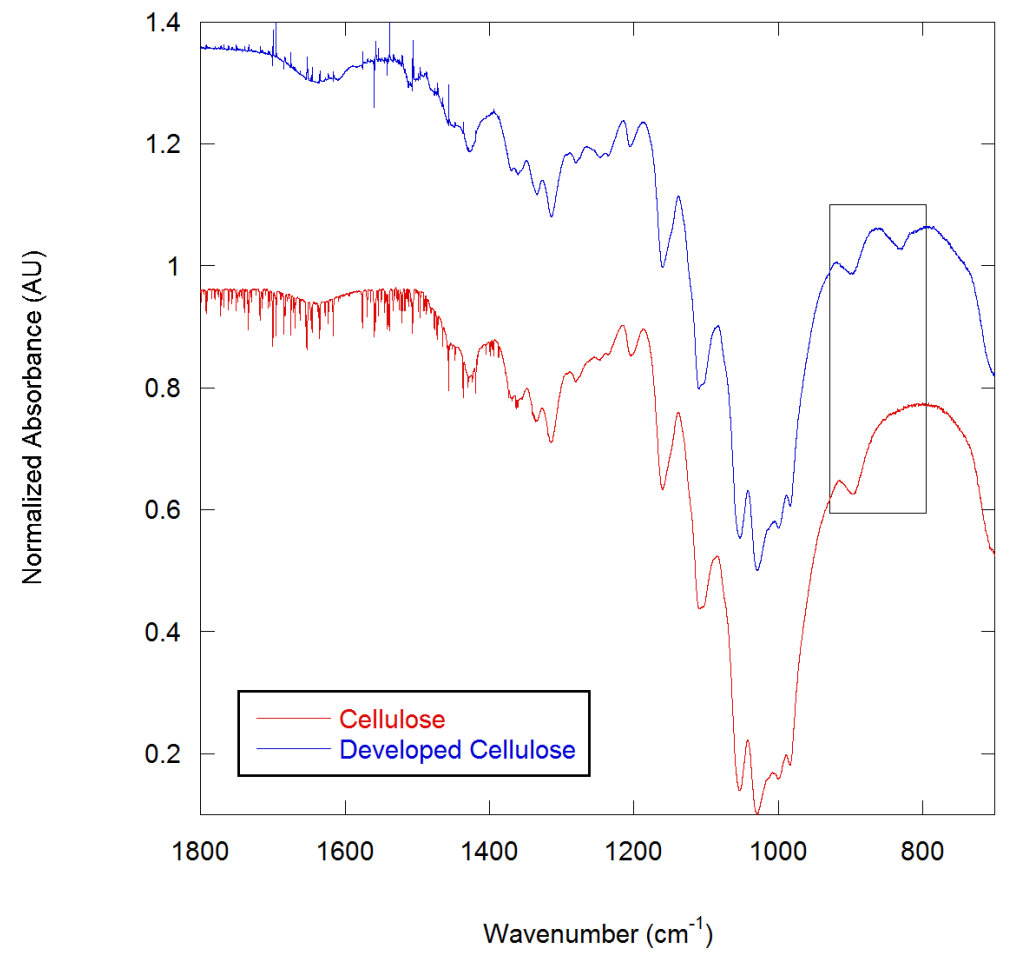

Figure 3.2.1.: A FTIR spectrum of (bottom) cellulose found in the untreated chromatography paper, and (top) developed (acetone, IPA washed) paper used in the production of microPADs. 
When discussing the FTIR spectra of SU-8 and SU-X microPADs a couple things should be kept in mind: (1) The spectra is dependent on the SU-X thickness and penetration of the microPAD at a given point. If the microPAD was not processed correctly and a thick SU-X film remains on the surface of the paper, the FTIR spectra may not show traces of cellulose; and (2) depending on the intensity of the molecular vibrations, the carbon-oxygen stretching of the epoxy rings may be present as the cis and/or trans stretch.

In Figure 3.2.2 we see the FTIR spectrum of an SU-8 impregnated microPAD before formation of the photoacid initiator. Two peaks stand out in the pre-exposure spectrum:

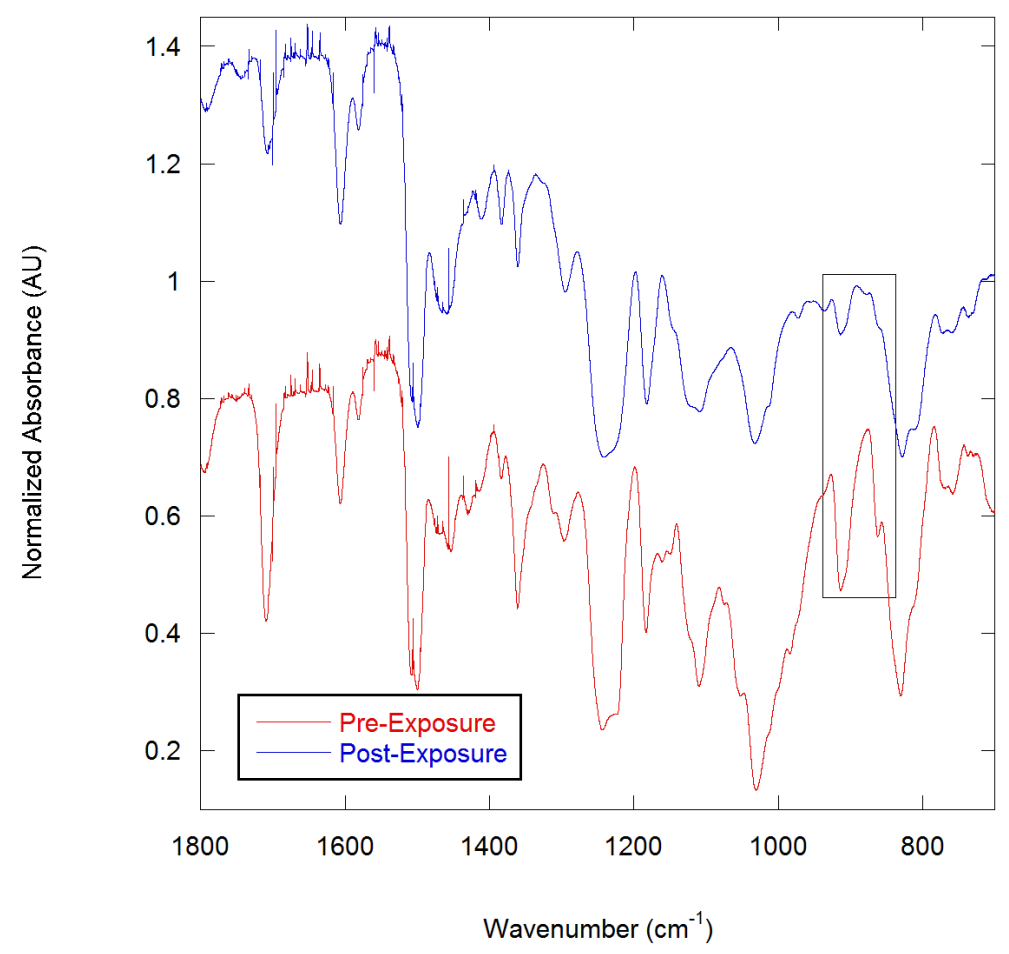

Figure 3.2.2.: A FTIR spectrum of (bottom) $40 \%$ (w/w) SU-8 impregnated paper before exposure, and (top) after exposure, in open environment.

a strong carbon-oxygen stretch of the trans substituted epoxy ring at $910 \mathrm{~cm}^{-1}$ and a less intense carbon-oxygen stretch of the cis substituted epoxy ring at $861 \mathrm{~cm}^{-1}$. In the postexposure spectrum, the trans substituted epoxy's carbon-oxygen stretch remains present with this spectrum, indicating that a portion of the SU-8 epoxy functionality remains. However, the intensity of this peak at $910 \mathrm{~cm}^{-1}$ is dramatically reduced, which suggests that 
the polymerization was successful but not complete. For comparison, Figure 3.2.3 presents the spectra of a portion of the microPAD which did not visually appear to polymerize. The

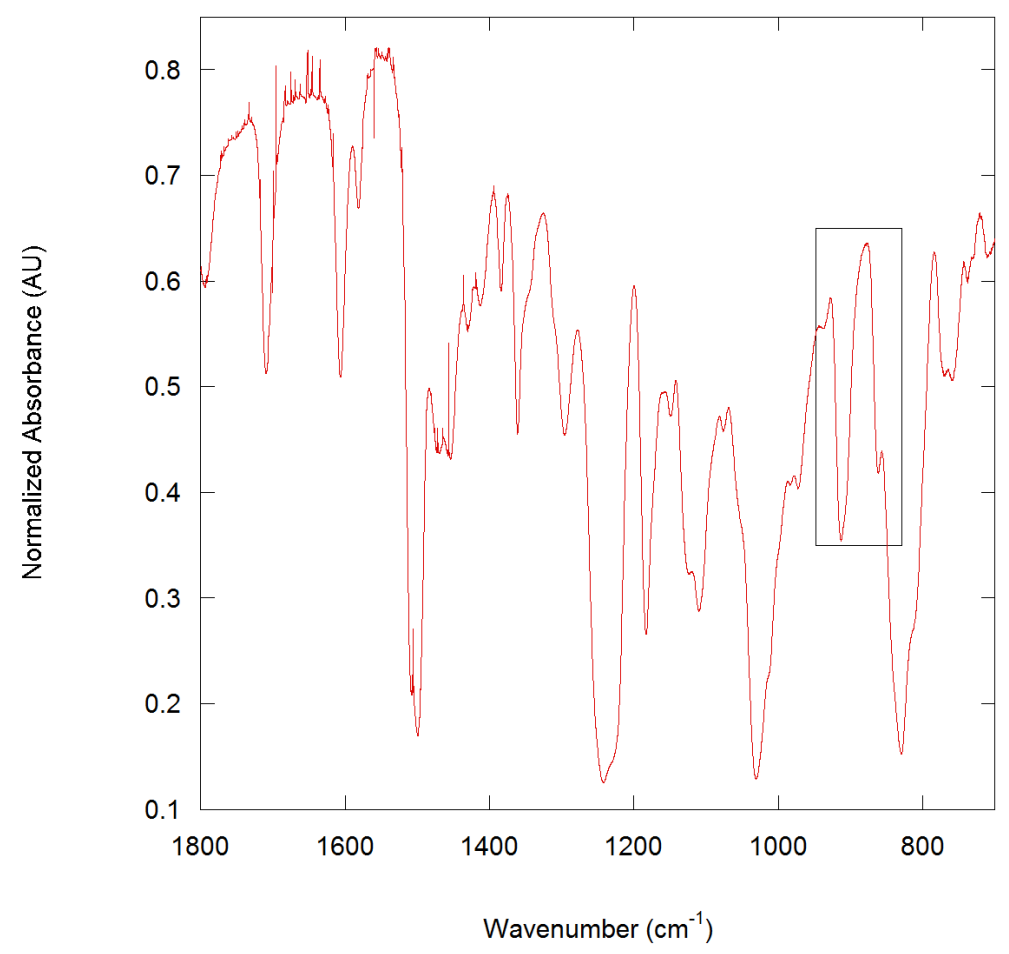

Figure 3.2.3.: A FTIR spectrum of an uncrosslinked portion of $40 \%$ (w/w) SU-8 impregnated paper after exposure, in open environment.

stretches of the epoxy ring here are akin to those featured in Figure 3.2.2, the pre-exposure impregnated microPAD. These spectra demonstrate that the success of the polymerization can be determined visually by a change in color and opacity of the paper and also show that the exposure process needed to be improved if the entire portion of the working microPAD was to be polymerized. Finally, Figure 3.2.4 gives the FTIR spectrum of the microPAD after both the post-exposure bake and hard bake. It is not clear what stretches are present at 877 and $932 \mathrm{~cm}^{-1}$, it suffices to say that these are not epoxy stretches suggesting (given the previous spectra) that the polymerization has gone to completion after the hard bake.

With the photolithographic process finalized, the effect of SU-8 weight percent concentration on the FTIR spectra was explored. Figure 3.2.5 displays a stacked plot of FTIR spectra for paper patterned with SU-8 in concentrations ranging from $40 \%$ (w/w) SU-8 
to $0 \%(w / w)$ SU-8 . Not surprisingly, the lower the SU-8 concentration, the more akin the spectrum is to the spectrum of cellulose (Figure 3.2.1). This result proved to be problematic when attempting to confirm the polymerization of low SU-X concentrations in paper, because not only was there no visible color or opacity change, there was also no apparent change in the FTIR spectra. Therefore, to determine the success of these low SU-X concentration microPADs an alternative method was used which is described in Section 3.2.2.

As discussed in Section 3.1.2, several alternate matrices were used with microPADs to compare the matrix effect different polymers had on the shelf life of an enzyme on microPADs. For PS and NC matrices, FTIR were not taken as premade polymer was only dissolved in solution and applied to the microPAD. Figure 3.2.6 shows a representative FTIR spectrum of a $40 \%(w / w)$ SU-8, $4 \%(w / w)$ PS microPAD.

\subsubsection{Microfluidic Paper-Based Analytical Device Hydrophobicity}

Polymers in general can be considered hydrophobic due to their long carbon backbones. By chemically incorporating varying functionality into the polymer backbone or sidechains this hydrophobicity can be modified to a great extent. However, hydrophobicity can also be affected by the physical modification of a surface. In an effort to apply this to paper, several processes were tested on SU-X impregnated paper. Before describing the results of these tests it should be noted that the transition from hydrophobic to hydrophilic SU-X impregnated paper will be reported here as a range of SU-X weight concentrations due to an inherent variation in individual microPAD and paper processing. This ultimately stems from having a processing system that requires bulk solution processing, as well as inconsistent and manual processing.

As it stands the hydrophobic-hydrophilic transition of SU-8 falls in the range of $2.5 \%$ (w/w) SU-8 to $5.0 \%(w / w)$ SU-8. Of 10 treated paper squares with $2.5 \%(w / w)$ SU-8, $80 \%$ absorbed the $3 \mu \mathrm{L}$ of water within 5 minutes; the remaining paper squares absorbed 


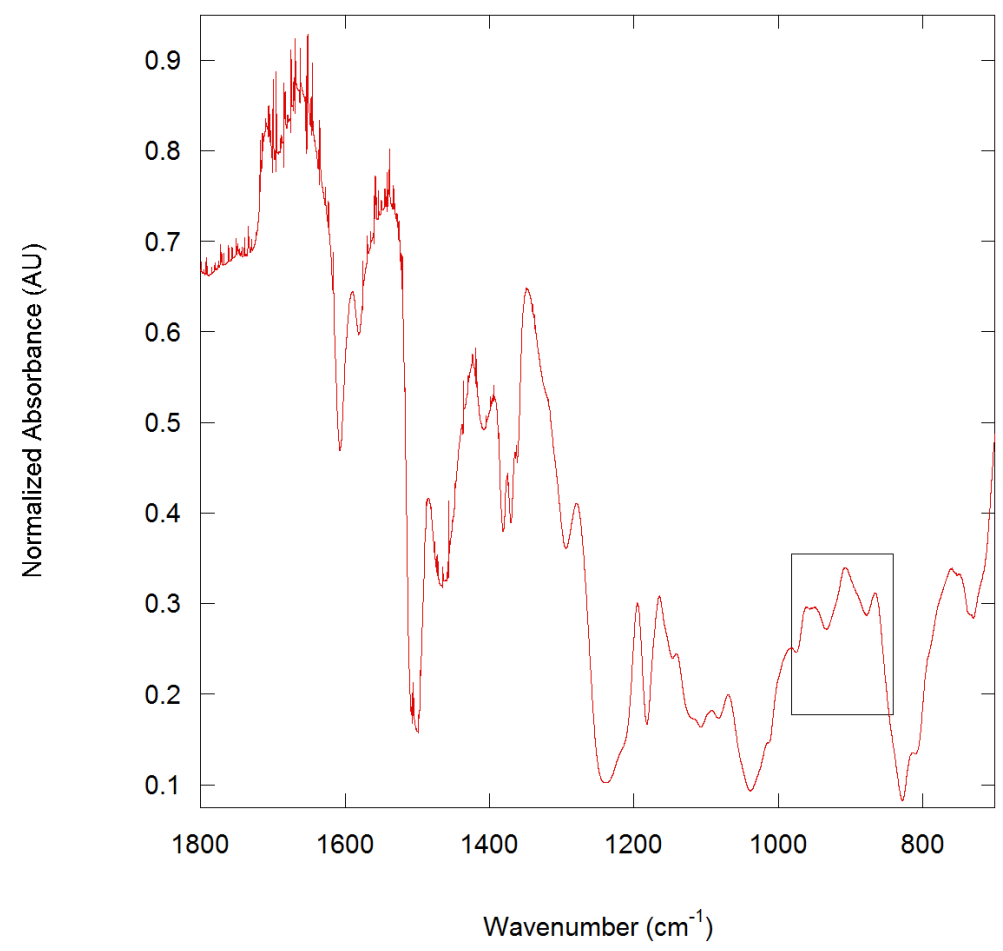

Figure 3.2.4.: A FTIR spectrum of $40 \%$ (w/w) SU-8 after the hard bake.

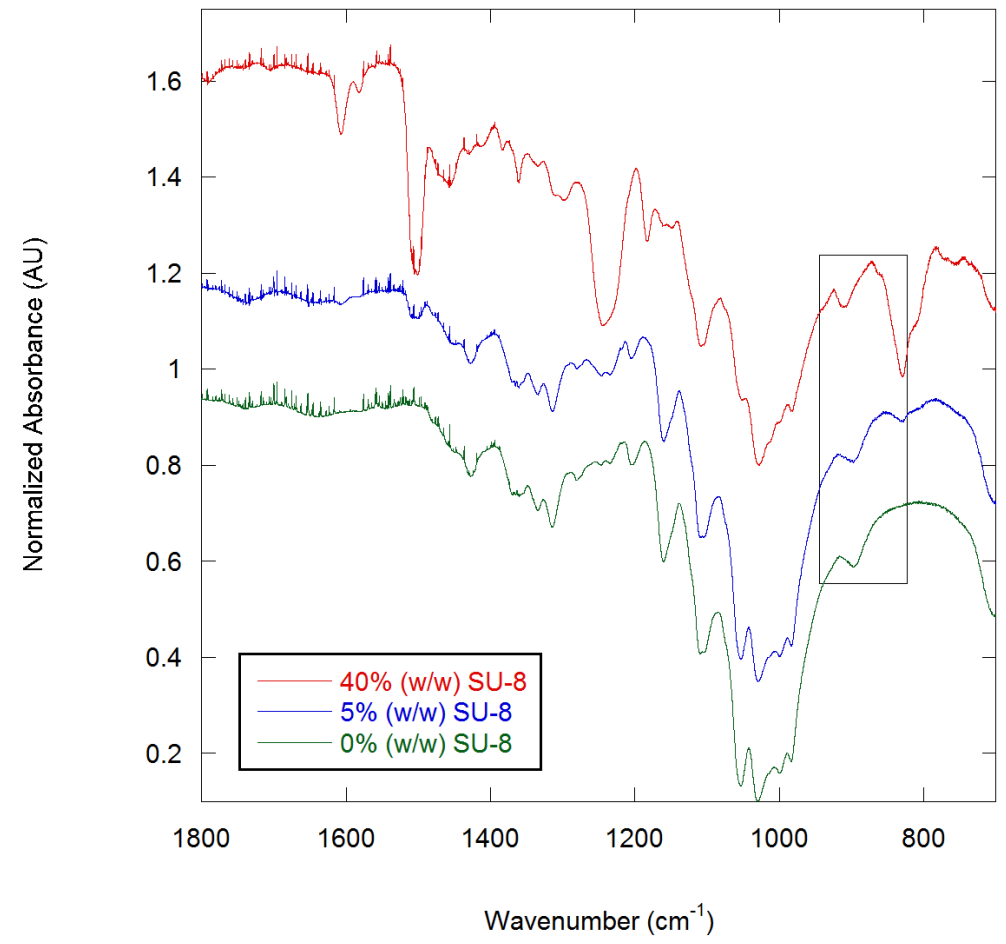

Figure 3.2.5.: Stacked FTIR spectra of the various SU-8 concentrations used: (top) $40 \%$ (w/w) SU-8, (middle) 5\% (w/w) SU-8, (bottom) 0\% (w/w) SU-8. 
the water in more than 5 minutes. Of 10 treated paper squares with $5.0 \%(\mathrm{w} / \mathrm{w}) \mathrm{SU}-$ $8,30 \%$ absorbed the aliquot of water after 5 minutes, the remainder failed to absorb or incompletely absorbed the water within the observation time. SU-8 concentrations at and below $1.3 \%(\mathrm{w} / \mathrm{w}) \mathrm{SU}-8$, immediately absorbed the complete aliquot of water while SU-8 concentrations at and above $10 \%(\mathrm{w} / \mathrm{w}) \mathrm{SU}-8$ failed to absorb into the paper before completely evaporating. The above hydrophobic-hydrophilic transitions describe the behavior of microPADs prepared from the processing described in Section 2.2.3.

The hydrophobic-hydrophilic transition for paper treated with SU-8 containing no photoinitiator was much broader and more highly variable. This was because the complete processing of microPADs, even without initiator, was typically performed with a development step in acetone; however, this step and the wash times associated with it were varied to determine if the hydrophobic-hydrophilic transition could be narrowed to a predictable range. The results of these tests showed a range of hydrophilicity from $0.63 \%(w / w) S U-8$ to $5.0 \%(\mathrm{w} / \mathrm{w}) \mathrm{SU}-8$. The length and total agitation of the development in acetone can be correlated to this range, in that longer development in acetone and more agitation made the microPAD with higher SU-8 concentrations more hydrophilic. For example, microPADs containing 5.0\% (w/w) SU-8 and washed in an acetone bath for 30 minutes were found to be completely hydrophilic to the testing method used (all 10 squares absorbed water in less than 5 minutes). This is not surprising as it is expected that the development process removes uncrosslinked SU-8 from the microPAD. More surprising was that rinsing the test microPADs with $10 \mathrm{~mL}$ of acetone through a disposable pipette would drastically affect the expected hydrophobicity of all concentrations of SU-8 in an unexpected manner. For example, of 10 test papers treated with $5.0 \%(\mathrm{w} / \mathrm{w}) \mathrm{SU}-8$ and rinsed with the latter method, $40 \%$ were found to absorb the aliquot of water within 5 minutes, $30 \%$ were found to absorb the aliquot of water in more than 5 minutes, and the remaining $30 \%$ failed to absorb the water into the microPAD. Without the development step, the hydrophobicity of the microPADs containing uncrosslinked SU-8 was much more predictable. At and 
below $0.63 \%$ (w/w) SU-8 the microPADs were found to be completely hydrophilic. Above $2.5 \%(\mathrm{w} / \mathrm{w})$ SU-8 the microPADs were completely hydrophobic and did not absorb water. MicroPADs prepared with $1.3 \%(\mathrm{w} / \mathrm{w})$ SU-8 and $2.5 \%(\mathrm{w} / \mathrm{w})$ SU-8 was entirely batch dependent with hydrophobicity due to the total SU-8 delivered to the microPAD, most microPADs within this range of SU-8 concentrations would absorb water within or after 5 minutes.

Other conditions such as pre-bake, post-bake, hard-bake temperatures and total times, as well as IPA wash time failed to affect hydrophobicity of crosslinked SU-8 microPADs to a measurable extent. Of these conditions, only IPA washing was observed to affect the properties of the surface of the microPAD by apparently increasing the relative surface energy to allow more complete wetting. However, to better probe this effect, contact angle measurements should be taken. None of the other mentioned conditions appeared to affect the hydrophobicity of any microPADs of a given SU-8 concentration, assuming crosslinking was successful. Incomplete or unsuccessful crosslinking tended to result in microPADs with complete hydrophilicity for all concentrations of SU-8 due to the development removing the SU-8 from the microPAD.

An additional attempt was made to increase the permeability of the SU-8, in order to enhance reagent delivery. The SU-8 films incorporated 4\% (w/w) PS of two molecular weights (800-5000, and 30000) into 40\% (w/w) SU-8 during the photolithographic process, and the PS was removed during the development step with acetone. The results of these tests were not found to be promising, as the surfaces appeared to become more hydrophobic. Ultimately, this processing was disregarded in lieu of better characterizing the shelf life of enzymes with varying SU-X concentrations. 


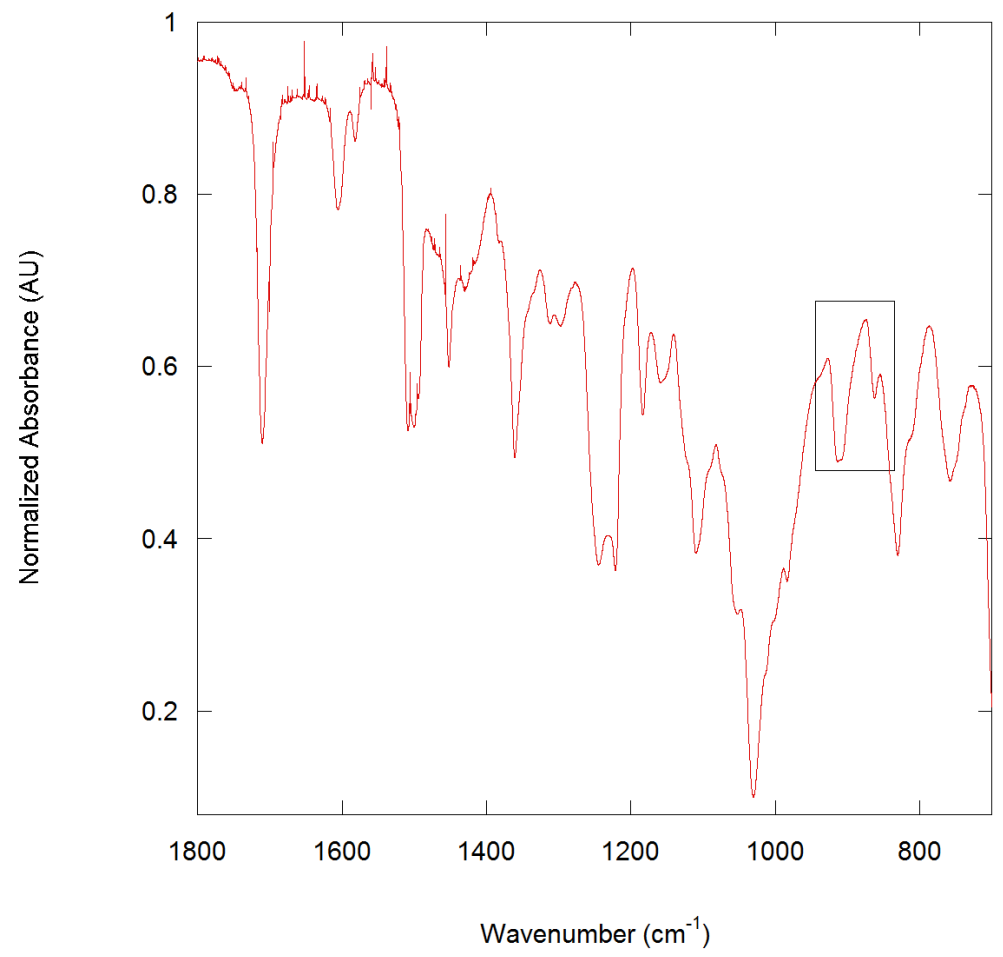

Figure 3.2.6.: A FTIR spectrum of $40 \%$ (w/w) SU-8 with $4 \%$ (w/w) PS post-development. 


\subsection{Enzyme Survival - Microfluidic Paper-Based Analytical Device Shelf Life}

Ultimately, the processing, conditioning, and characterization of the microPADs discussed thus far was to define to what extent an enzyme can survive on paper when treated in a specific way. Discussed below is the extent of the enzyme shelf life for microPADs treated under a variety of conditions. Each condition described below will be compared to three controls: (1) enzyme in $1 \mathrm{X}$ PBS and IPA, stored at both $4{ }^{\circ} \mathrm{C}$ and room temperature (Figure 3.3.1); (2) unstabalized enzyme deposited directly on paper (Figure 3.3.2a); and (3) enzyme deposited after trehalose was deposited directly on paper (Figure 3.3.2b; discussed in 1.1.2).

Figure 3.3.1 shows the first of the controls, solution stabilized enzyme (SSE), for two different enzyme solutions. Here, a large range of relative signal from both the 1X PBSHRP enzyme solution and the IPA-HRP solutions was observed. This variation could be due to two possible reasons: (1) variations in the temperature of the enzyme solution each time the assay was performed, and (2) variations in the substrate concentration delivered to the enzyme. Regardless of the approximate maximum difference of 30 percent signal, the enzyme activity was assumed constant for the duration of the shelf life experiments because there is no clear decline in signal over time. The variation in signal should be kept in mind when observing the trends for enzyme activity of HRP on microPADs as presented below, because this would contribute to the standard deviation of each datum in the shelf life experiments; however, due to the scope of this project, this variable is ignored in lieu of observing the physical ability of SU-X, or other polymer matrices, to prevent the clear decline of enzyme activity over time.

Figures 3.3.2 show the shelf life of unstabalized 1X PBS-HRP and trehalose stabilized 1X PBS-HRP on paper. Both figures show that HRP rapidly loses activity and the enzyme is trending towards being completely denatured after two to three days. This shelf life is less than ideal for microPAD applications, where devices should ideally have a shelf life with predictable activity for 6 months to a year. In total, the control experiments performed 
suggest that HRP has the potential to demonstrate, in a short time period, the efficacy of different stabilizing treatments for enzymes on paper; additionally, these experiments show the experimental setup to be a good model system for studying the stability of enzymes on paper.

\subsubsection{Unexposed SU-8 Shelf Life}

Initial shelf life tests were performed during the processing optimization of SU-X. These tests utilized the entirety of the photolithographic processing steps discussed in Section 2.2.3, with the exception of the exposure via sunlight or UV lamp. To ensure crosslinking did not occur, the photoinitiator, triarylsulfonium hexafluorophosphate salts, was not added to the SU-X solution mixtures. Additionally, the effects of the processing order on paper and SU-8 addition were explored. Initial testing revealed that the processing order of the SU-8 microPADs had little effect on enzyme survival over a period of 10 days. A more important condition is the total concentration of SU-8 present on the microPAD, as well as the presence of the disaccharide trehalose in addition to SU-8. With this in mind experiments were performed testing different concentrations $(0.63 \%, 1.3 \%, 2.5 \%$, and $5.0 \%(\mathrm{w} / \mathrm{w}))$ of SU-8 with both trehalose and no trehalose. There is a clear trend of decreased activity over the 17 day period (Figure 3.3.3 ). A trend is seen that at higher concentrations of SU-8, more stabilization occurs; however, at the highest concentration $(5.0 \%(\mathrm{w} / \mathrm{w}))$ this stabilizing effect appears to diminish. Figure 3.3.4 represents a composite image of the $2.5 \%(\mathrm{w} / \mathrm{w}) \mathrm{SU}-8$ assay results scanned thirty minutes after the assay was performed, here we see a clear decline in the intensity of the precipitate product over time. Overall, these results were promising when compared to the controls; however, this trend of stability needed to be explored in more depth. Additionally, issues arise when reproducing specific microPADs as SU-8 monomer is susceptible to solvents, and unexposed SU-8 can also be tacky if the film is thick enough. Unexposed SU-8 is therefore not suitable for long term use with 2-D microPADs, in an open environment. 


\subsubsection{Alternative Matrices Shelf Life}

Alternative polymer matrices explored for HRP shelf life on paper included nitrocellulose (NC) and polystyrene (PS), both at 2.5\% weight percent. Figure 3.3.5 shows the result obtained from these shelf life experiments. Half of the microPADs used were also primed for reagent delivery by soaking in a $\mathrm{DI} \mathrm{H}_{2} \mathrm{O}$ bath. For all but the $\mathrm{NC}$ microPADs, there was no difference in the results between the primed and unprimed microPADs. This is significant for SU-X prepared microPADs, because at lower SU-X concentrations this method may be used to increase throughput of prepared microPADs when delivering reagents to the device since reagents that wick into the paper dry faster than a drop of solution not permeating into the microPAD.

Figure 3.3.5 additionally displays stabilizing effects present with all polymer matrices to varying degrees. PS had the smallest effect on signal production, with NC performing only slightly better by losing 100 percent enzyme signal in 10 days. After the same 10 day period, SU-8 retained approximately 20 percent signal. With this in mind, SU-8 and SU-X stabilization was explored in more detail in subsequent sections. An interesting phenomena was observed over the initial days of the experiment. Here a sharp decrease in the enzyme activity is seen during the first two days. Followed by a lower rate of the loss of enzyme activity for the remainder of the experiment. It is likely that this initial sharp loss of activity corresponds to the loss of water from the enzymes surroundings, as this sharp decrease is reminiscent of the decline in activity from trehalose stabilized HRP, which relies heavily on adsorbed water to affect enzyme stabilization. After this sharp decline in activity, the mechanism of stabilization is likely singularly due to that of the polymer present on the microPAD. More testing is necessary to narrow down the specific means of enzyme stabilization. 


\subsubsection{SU-X Shelf Life}

MicroPAD containing SU-X, either "SU-4" or SU-8, were explored for low and high SU-X concentrations in microPADs. Low concentrations, as discussed in Section 3.2.2, typically resulted in microPADs which had better reagent loading and assay characteristics. However, higher and hydrophobic concentrations of SU-X showed greater promise for long term enzyme stability on microPADs.

\subsubsection{Low SU-X Concentrations}

Previous discussion in this section utilized low SU-X concentrations in many of the experiments due to the ease of processing and reagent loading. Drawing from these experiments and the microPAD shelf life shown in Figure 3.3.6, a relatively high variability at supposedly the same concentration of SU-8 delivered to the microPAD suggests inconsistencies in either the processing of the microPAD or different shelf-li ves for similar enzyme concentrations. The former is more probable as microPAD processing over the course of experiments was overall inconsistent. A summary of these experiments is given in Figure 3.3.7, here a range of approximately 20 percent total signal can be expected, no matter the initial signal of the microPAD or the rate of the loss of enzyme activity. This suggests that earlier experiments, performed before more consistent processing was used, should be repeated to establish appropriate trends.

Figure 3.3.6 compares low SU-8 concentration microPADs loaded with either 1X PBS diluted HRP or IPA diluted HRP. IPA assists with enzyme loading into the test zones of the microPADs for higher concentrations of SU-X. However, for low concentrations of SU-X, IPA is able to easily penetrate the wax barriers of a microPAD creating irregular patterns and assay test zones. This likely contributed to the variability of the signal from the IPAHRP assays. Alternatively, enzyme in IPA solutions could be denaturing at a higher rate, or the concentration of enzyme delivered to the microPAD from IPA could be affecting the stability of the enzyme. IPA-HRP assays will also consistently include a large dip in signal 
after the first day, despite the stability seen from enzyme in IPA (Figure 3.3.1). Overall, IPA-HRP appears to have worse performance on microPADs of low SU-8 concentration in comparison to $1 \mathrm{X}$ PBS-HRP for low concentrations of SU-8. An unexplored

\subsubsection{High SU-X Concentrations}

Higher SU-X concentration microPADs are increasingly hydrophobic and tend to lose the wicking characteristics of paper to a great extent. In order to retain microPAD functionality, high SU-X concentrations were tested under several different conditions: (1) with 1 X PBSHRP spotted on the microPAD and an assay performed with $50 \%$ (v/v) IPA in ABTS, and (2) with IPA-HRP spotted on the microPAD and assay performed with $50 \%(\mathrm{v} / \mathrm{v})$ IPA in ABTS. The latter condition is shown in Figures 3.3.8a and 3.3.8b, which shows that increasing the concentration of the polymer added to the microPAD increases the stability of HRP over time. This could be due to two possible reasons: (1) an increase in the total crosslink density present on the microPAD, or (2) an increase in the concentration of epoxide/ether functionality. These effects could increase the number of hydrogen bond acceptors on the surface and within the microPAD, which in turn could slow the evaporation of water from the device, thus keeping the enzyme hydrated for extended periods; they could produce a more enzyme-friendly surface for the enzyme to adsorb on to; or, in the case of entrapment, they could provide an effective lock on the enzymes quaternary/tertiary structure. Regardless of the mechanism of stabilization more initial epoxide functionality on the paper results in longer shelf life.

An experiment comparing the shelf life of microPADs produced with a set of $2.5 \%, 10 \%$, and $40 \%$ (w/w) SU-8 spotted with 1X PBS-HRP, a set of $2.5 \%, 10 \%$, and $40 \%$ (w/w) SU-8 microPADs spotted with IPA-HRP, as well as $40 \%$ (w/w) SU-4 microPAD spotted with 1X PBS-HRP and a 40\% (w/w) SU-4 microPAD spotted with IPA-HRP, was performed. The 1X PBS-HRP solutions were spotted to adsorb onto SU-X, while the comparative 
experiment was spotted with IPA-HRP to entrap within the SU-X. In all cases substrate delivery was by $50 \%(\mathrm{v} / \mathrm{v})$ IPA in ABTS. The results of this are seen in Figures 3.3.9.

Over at most a three day period, there is a decline in signal for nearly all devices; however, after this, an apparent stable signal is reached. At $40 \%(\mathrm{w} / \mathrm{w}) \mathrm{SU}-\mathrm{X}$ concentrations the initial dip in signal appeared only in IPA-HRP solutions, suggesting a change in stabilization at these transisitons. Lower concentrations of SU-X performed poorly, with the exception of $10 \%$ (w/w) SU-8 spotted with 1XPBS-HRP, losing all signal after the first day. Taking into account the previous experience with fabricating microPADs, it is likely that these microPADs did not have successful fabrications and SU-8 was completely removed during development. Larger absolute signals were seen for SU-4 microPADs, this is not an indication of overall enzyme stability because differences in the concentration of enzyme delivered with IPA-HRP is not taken into account. 


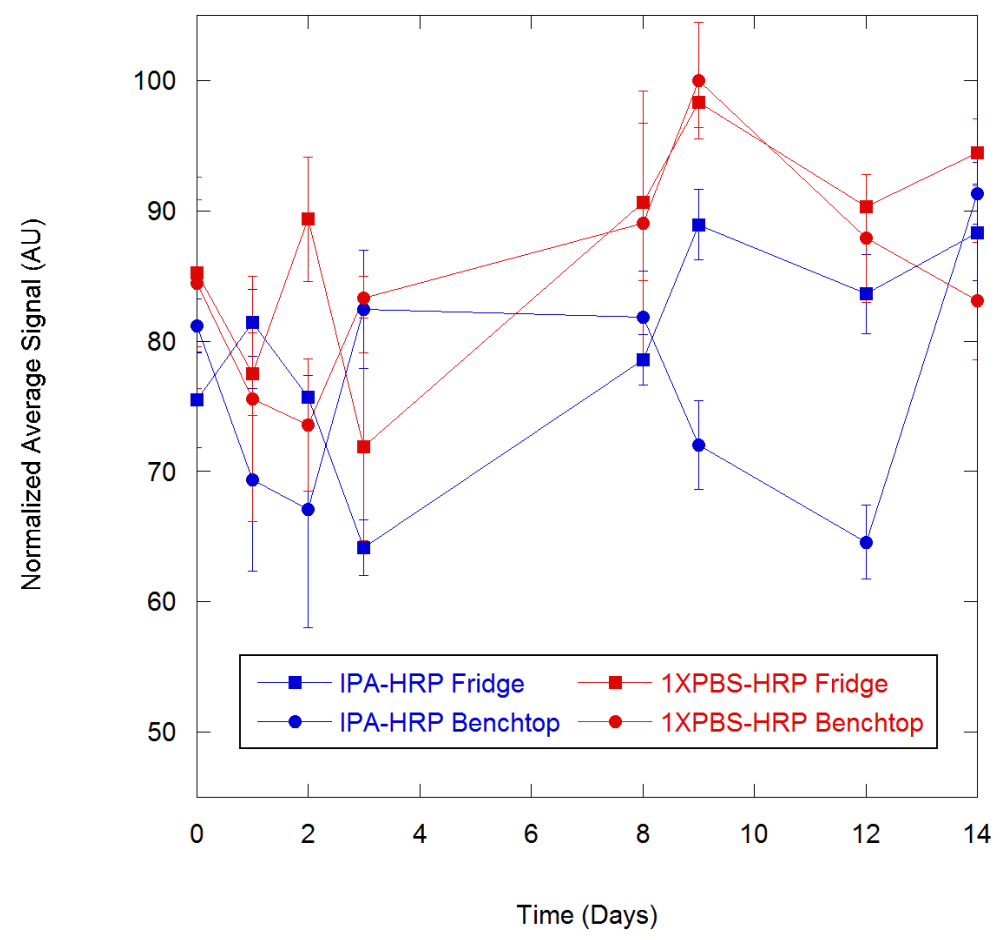

Figure 3.3.1.: Solution stabilized horseradish peroxidase, stored at (O) $4{ }^{\circ} \mathrm{C}$ and (ם) room temperature for (Blue) HRP in $1 \mathrm{X}$ PBS and (Red) HRP in IPA. 


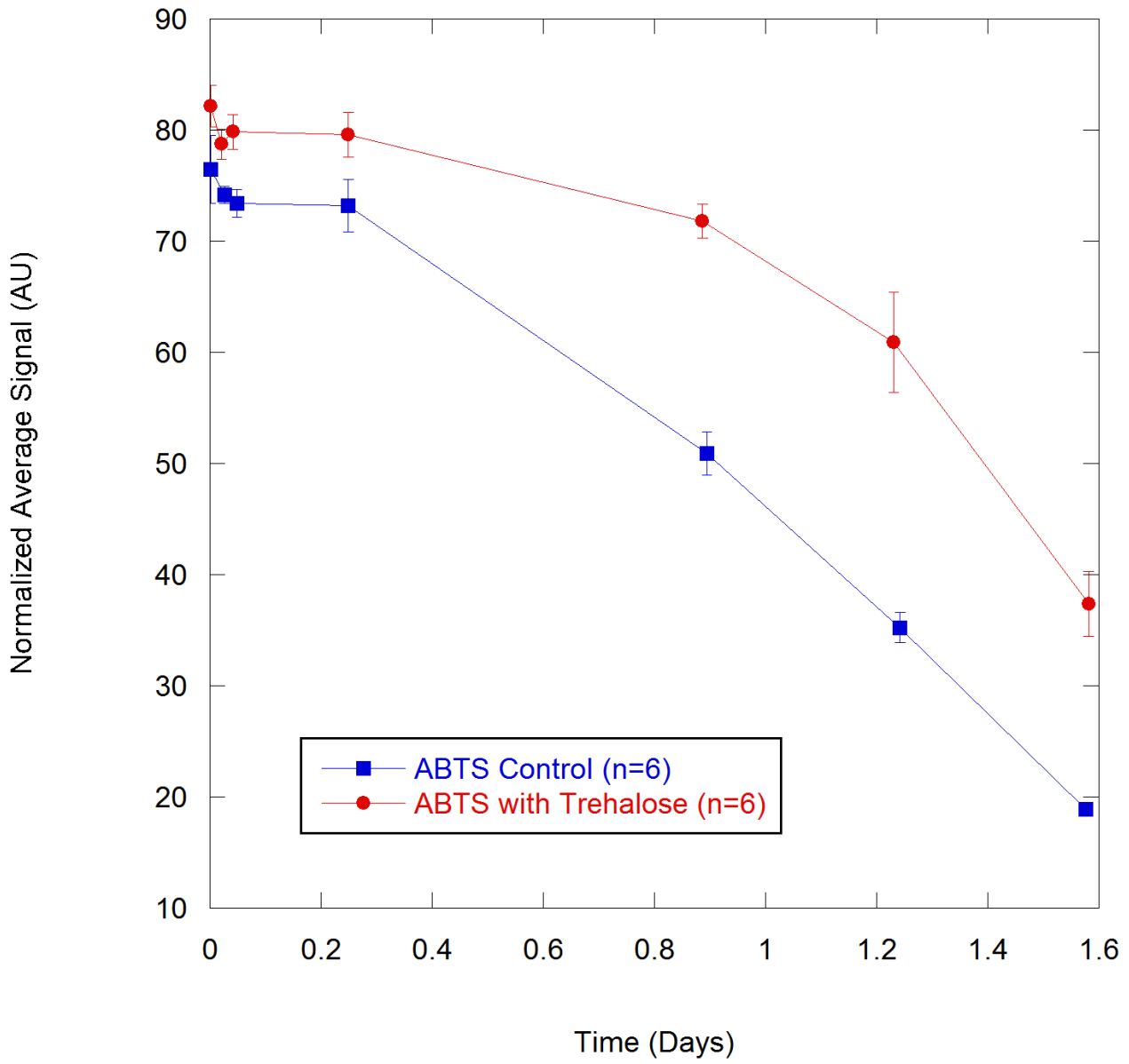

Figure 3.3.2.: HRP deposited to microPADs: $(\bullet)$ unstabalized HRP on paper, and (ם) trehalose stabilized HRP on paper. 


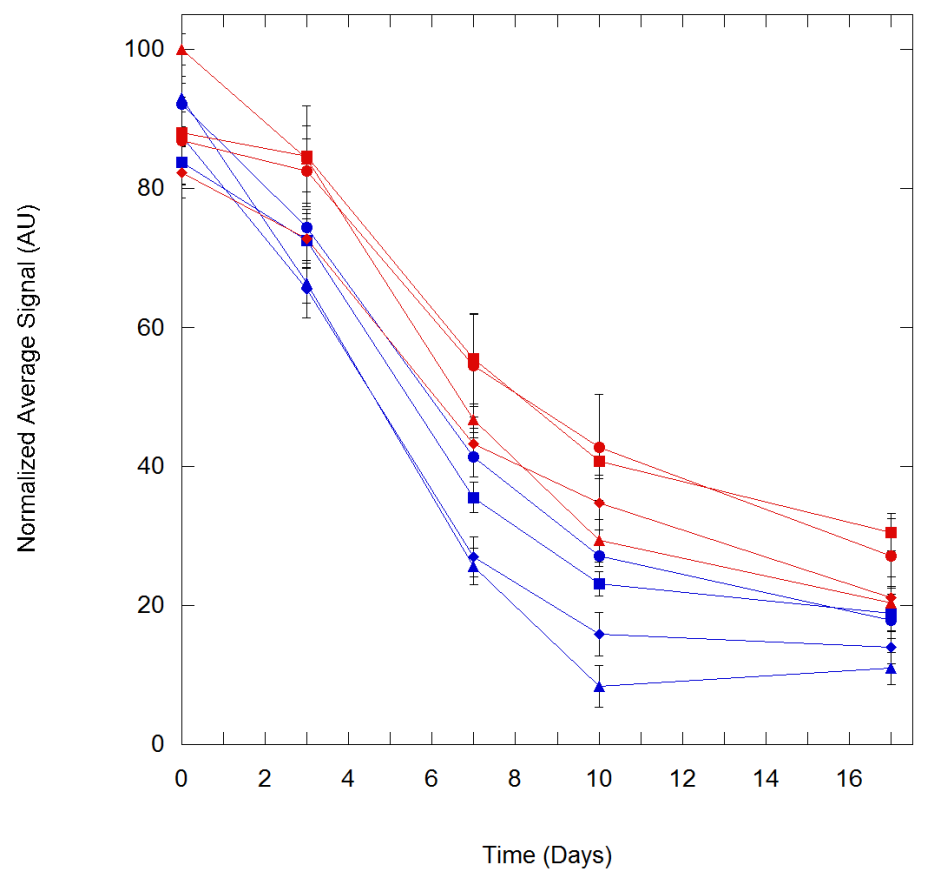

\begin{tabular}{|c|c|}
\hline - $0.6 \%(\mathrm{w} / \mathrm{w}) \mathrm{SU}-8$, no Trehalose & $\Rightarrow \quad 0.6 \%(\mathrm{w} / \mathrm{w})$ SU-8, with Treh \\
\hline$\longrightarrow 1.3 \%(\mathrm{w} / \mathrm{w})$ SU-8, no Trehalose & $\longrightarrow 1.3 \%(\mathrm{w} / \mathrm{w}) \mathrm{SU}-8$, with Trehalose \\
\hline$\longrightarrow 2.5 \%(\mathrm{w} / \mathrm{w})$ SU-8, no Trehalose & $\longrightarrow 2.5 \%(\mathrm{w} / \mathrm{w}) \mathrm{SU}-8$, with Trehalose \\
\hline$\longrightarrow 5.0 \%(\mathrm{w} / \mathrm{w}) \mathrm{SU}-8$, no Tre & $\longrightarrow 5.0 \%(\mathrm{w} / \mathrm{w}) \mathrm{SU}-8$, with Trehalose \\
\hline
\end{tabular}

Figure 3.3.3.: Shelf life for variable low concentrations of SU-8, both with and without trehalose for unexposed SU-8 without development.

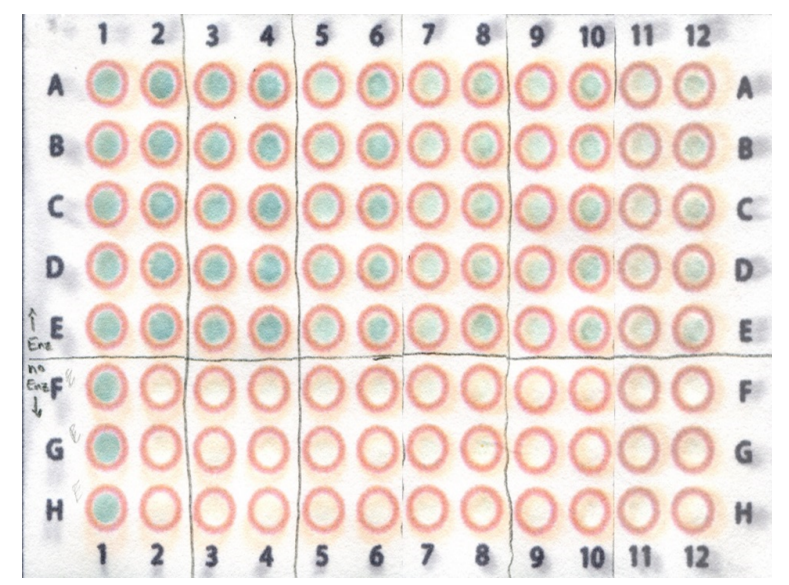

Figure 3.3.4.: Composite image of the results displayed in Figure 3.3.3. Odd numbered columns contain $1 \mu \mathrm{L}$ of $1 \mathrm{M}$ trehalose in addition to $2.5 \%(\mathrm{w} / \mathrm{w}) \mathrm{SU}-8$. Even numbered columns contain no trehalose, but retain the $2.5 \%$ (w/w) SU-8. Columns $1 \& 2$ represent day 0 results; columns $3 \& 4$ represent day 3; columns $5 \& 6$ represent day 7; columns 7 $\& 8$ represent day 10; $9 \& 10$ represent day 14; finally, columns $11 \& 12$ represent day 17 . Rows A through E include all reagents including enzyme. Rows F through $\mathrm{H}$ contain all reagents except enzyme. With the exception of column 1, rows F-H which do contain enzyme. 


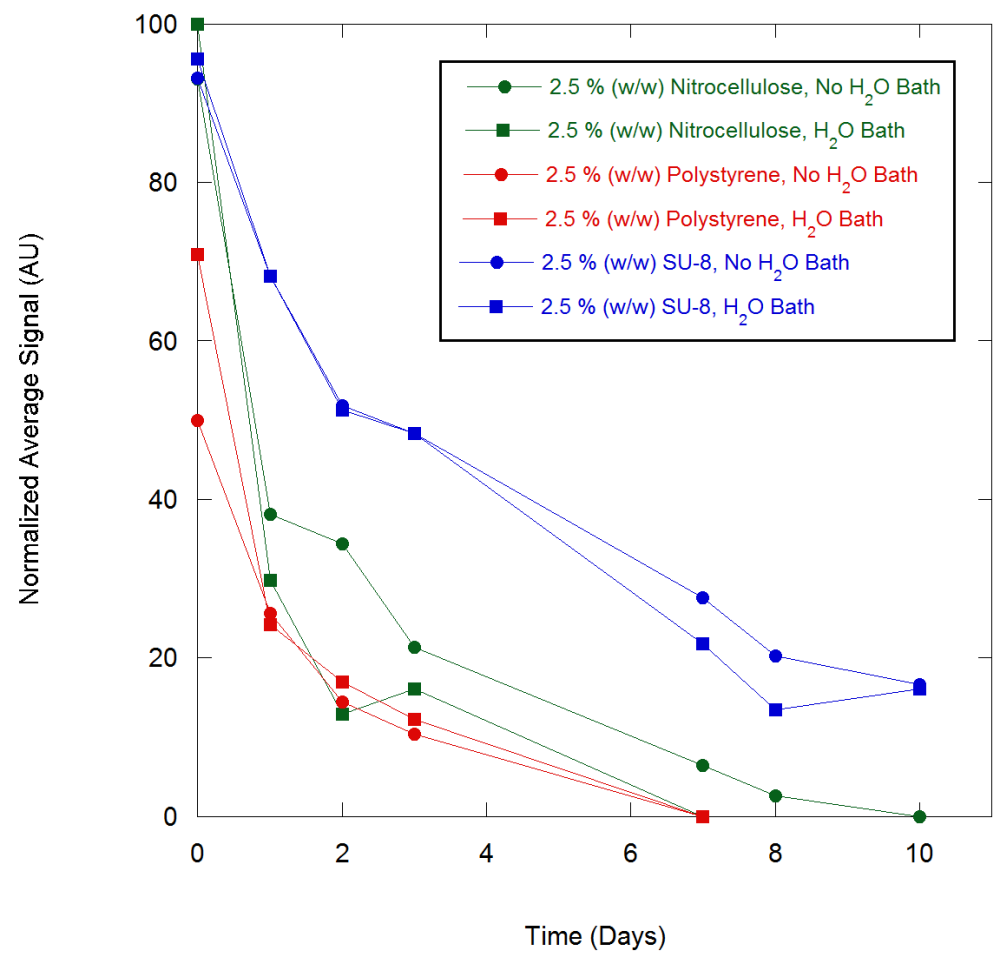

Figure 3.3.5.: MicroPADs containing (Blue) 2.5\% (w/w) SU-8, (Red) 2.5\% (w/w) PS MW 800-5000, and (Green) 2.5\% (w/w) NC both (O) presoaked with DI H2O before enzyme deposition, and (ם) enzyme deposited directly after polymer. 


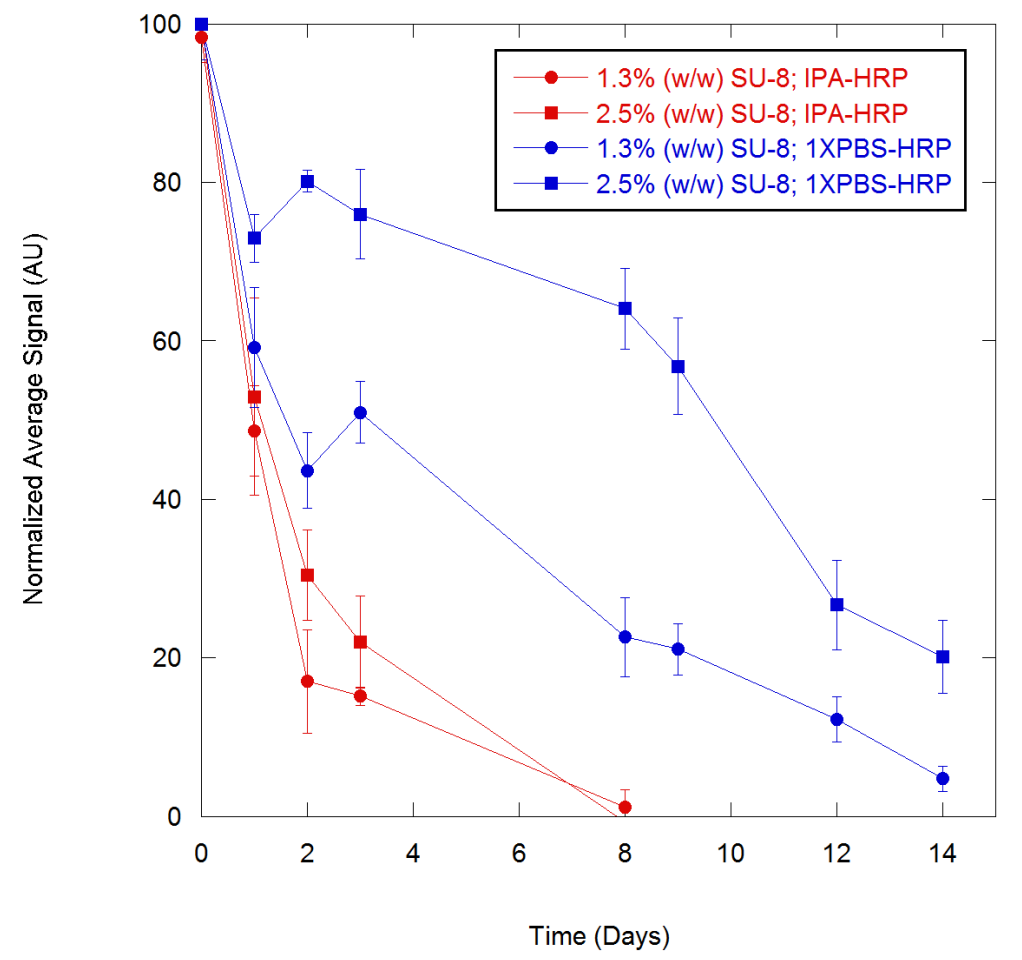

Figure 3.3.6.: MicroPADs impregnated with (๑) $1.25 \%(\mathrm{w} / \mathrm{w})$ SU-8 and (口) $2.5 \%(\mathrm{w} / \mathrm{w})$ impregnated with (Red) IPA-HRP and (Blue) 1X PBS-HRP. Assays were run with 50\% (v/v) IPA-ABTS. 


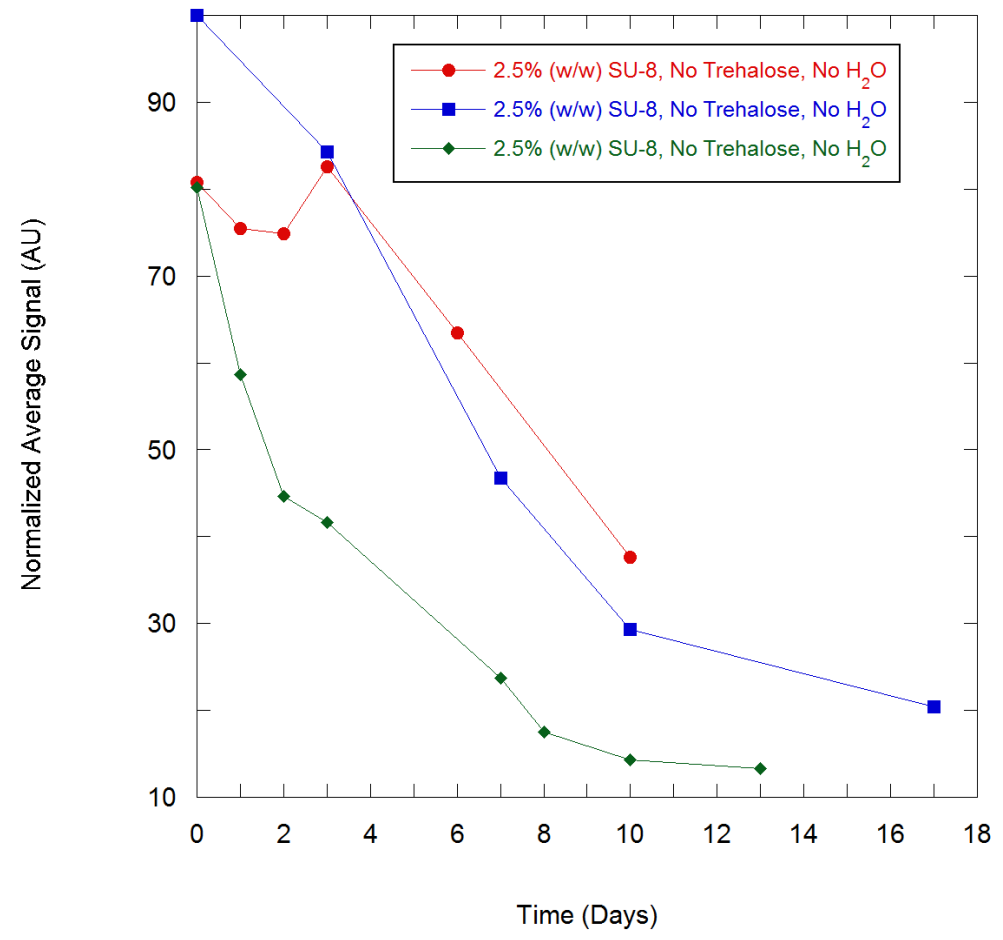

Figure 3.3.7.: Summary of low SU-8 concentrations performed over the course of the shelf life experiments. 

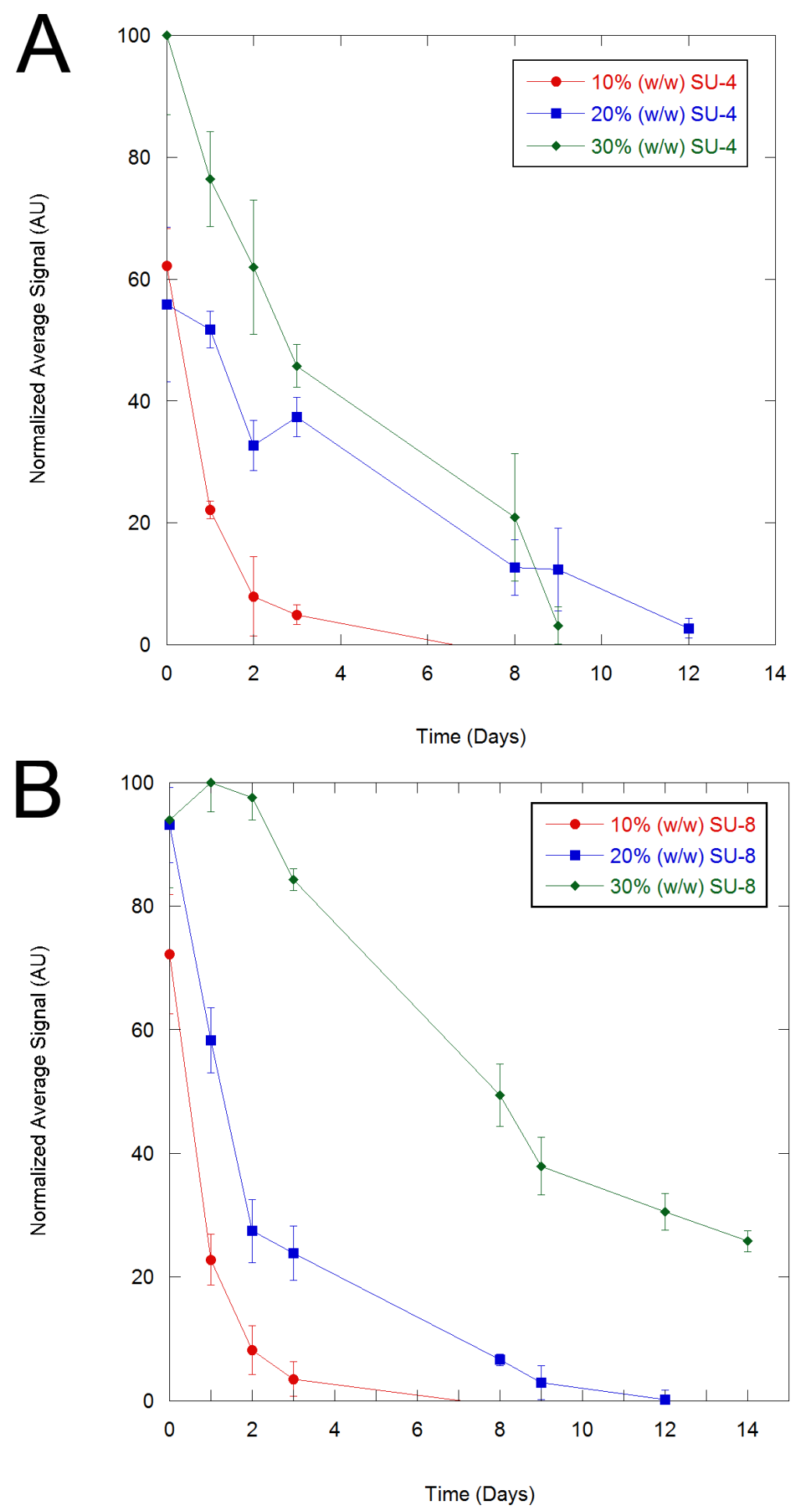

Figure 3.3.8.: Hydrophobic concentrations of SU-X on fully processed microPADs. Assays were run with 50\% (v/v) IPA-ABTS to avoid the hydrophobic effects of high SU-X concentrations: (a) microPADs with fully processed $(\bullet) 10 \%(\mathrm{w} / \mathrm{w}),(\boldsymbol{\square}) 20 \%$ $(\mathrm{w} / \mathrm{w})$, and $(\bullet) 30 \%(\mathrm{w} / \mathrm{w})$ "SU-4;" and (b) microPADs with fully processed $(\bullet) 10 \%$ (w/w), (匹) 20\% (w/w), and (•) 30\% (w/w) "SU-8." 


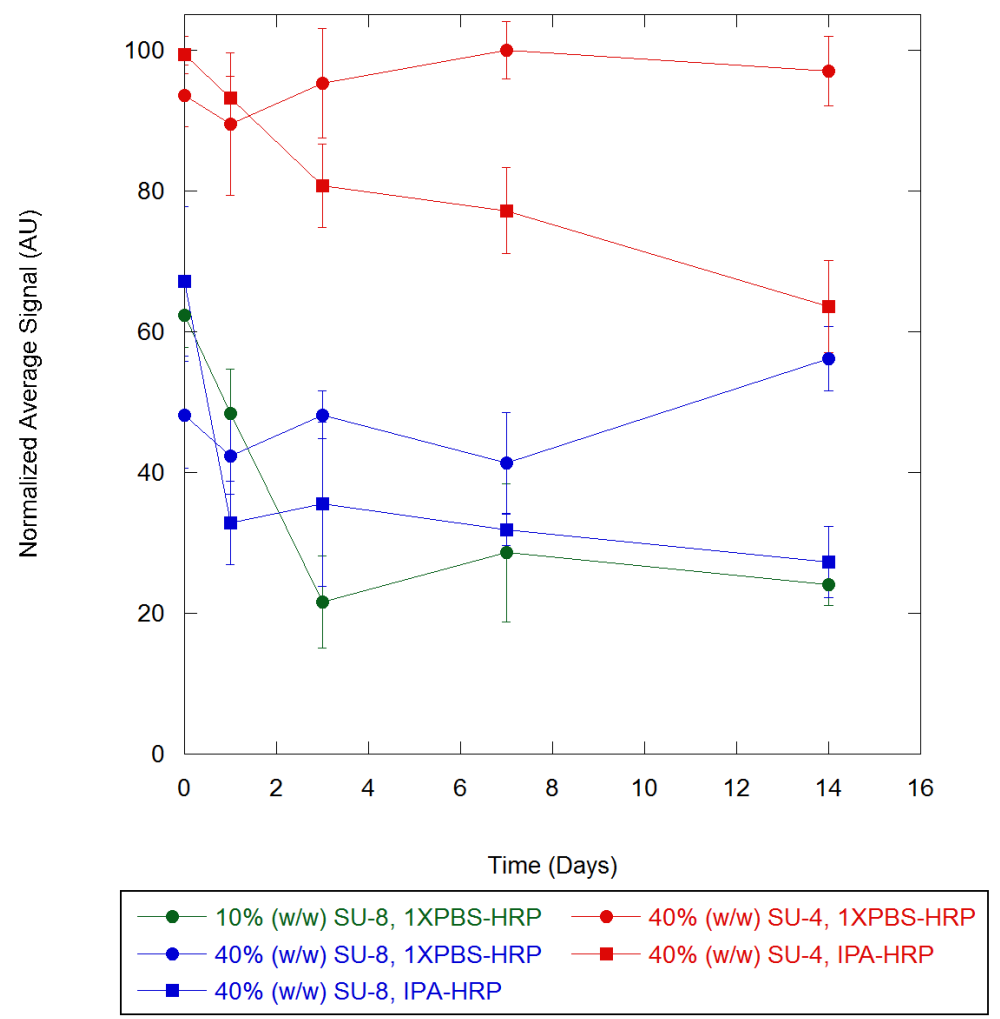

Figure 3.3.9.: Comparison of activity of high SU-X concentration microPADs spotted with (a) IPA-HRP, or (b) 1X PBS-HRP. 


\section{Conclusions}

The combination of wax printing and photolithography was achieved with SU-8 on microPADs. Improvements and challenges still remain, these include the use of solvents in the photolithography process, which dissolve wax barriers. The use of isopropyl alcohol (IPA) as a reagent delivery medium complicates the use of wax printing as well, as it allows for penetration of the wax barriers by reagents. While wax printing remains a cost-effective standard for microPAD production, its incorporation into other fabrication processes is not recommended - particularly with photolithography. In lieu of producing hydrophobic barriers, other fabrication methods could be used. For example, Teflon-barriers would remain impervious to IPA and any solvent likely used in the photolithographic process. ${ }^{53}$

Characterization of the photolithographic process as well as characterization of the produced microPADs ability to accept aqueous reagent delivery was performed. FTIR was found to be an acceptable method to qualitatively follow the crosslinking of SU-X by means of the disappearance of the stretches for epoxide moieties. Future work on this topic would further characterize both the amount to SU-X delivered to an area of microPAD and the mechanism of enzyme stabilization on SU-X. These experiments could potentially be performed with thermogravimetric analysis and surface imaging (e.g. atomic force microscopy, scanning electron microscope) respectively.

Finally, SU-X was found to improve the shelf-life of microPADs treated with a variety of concentrations of SU-8 and SU-4. The most promising results were shown for higher concentrations of SU-X; however, at these high concentrations, reagent delivery to the devices becomes problematic. IPA was found to be an effective medium for delivering a substrate when diluted 1:1 ratio by volume with the aqueous substrate solution. Creating IPA diluted samples complicates the potential application of microPADs containing stabilizing SU-X. Further exploration into the effect of SU-X on enzyme shelf-life is required, specifically with regard to substrate addition to the microPAD. Several avenues of exploration may 
be treating the SU-X containing paper chemically, or with corona/plasma. Regardless, the application of SU-8 and other equivalent epoxy novolac resins appears promising and should continue to be explored as a potential enzyme stabilizing platform on paper. 


\section{REFERENCES}

[1] McNeil Jr., D. G. Far From Any Lab, Paper Bits Find Illness. 2011; http://www . nytimes. com/2011/09/27/health/27paper.html.

[2] Peeling, R.; Holmes, K. K.; Mabey, D.; Ronald, A. Sexually transmitted infections 2006, 82 Suppl 5, v1-6.

[3] Pelton, R. TrAC Trends in Analytical Chemistry 2009, 28, 925-942.

[4] Zhao, W.; van der Berg, A. Lab on a chip 2008, 8, 1988-91.

[5] Giddings, J. C.; Keller, R. A. Advances in Chromatography, Volume 1; M. Dekker: New York, 1965.

[6] Adams, J. M.; Dolin, P. A. Printing Technology; Delmar Cengage Learning: Florence, KY, 2002; p 542.

[7] Price, C. P. 2001, 1285-1288.

[8] Martinez, A. W.; Phillips, S. T.; Whitesides, G. M.; Carrilho, E. Analytical chemistry $2010,82,3-10$.

[9] Yager, P.; Domingo, G. J.; Gerdes, J. Annual review of biomedical engineering 2008, $10,107-44$.

[10] Chin, C. D.; Linder, V.; Sia, S. K. Lab on a chip 2012, 12, 2118-34.

[11] Sia, S. K.; Kricka, L. J. Lab on a chip 2008, 8, 1982-3.

[12] Yager, P.; Edwards, T.; Fu, E.; Helton, K.; Nelson, K.; Tam, M. R.; Weigl, B. H. Nature 2006, 442, 412-8.

[13] Martinez, A. W.; Phillips, S. T.; Carrilho, E.; Thomas, S. W.; Sindi, H.; Whitesides, G. M. Analytical chemistry 2008, 80, 3699-707. 
[14] Roine, R.; Ohinmaa, A.; Hailey, D. CMAJ : Canadian Medical Association Journal $2001,165,765-71$.

[15] Yagoda, H. Industrial \& Engineering Chemistry Analytical Edition 1937, 9, 79-82.

[16] Müller, R. H.; Clegg, D. L. Analytical Chemistry 1949, 21, 1123-1125.

[17] Martinez, A. W.; Phillips, S. T.; Butte, M. J.; Whitesides, G. M. Angewandte Chemie (International ed. in English) 2007, 46, 1318-20.

[18] Li, X.; Ballerini, D. R.; Shen, W. Biomicrofluidics 2012, 6, 11301-1130113.

[19] Lorenz, H.; Despont, M.; Fahrni, N.; LaBianca, N.; Renaud, P.; Vettiger, P. Journal of Micromechanics and Microengineering 1997, 7, 121-124.

[20] Commons, W. Comparison Positive Negative Tone Resist. 2013; https: //en.wikipedia.org/wiki/Photoresist\#mediaviewer/File:Comparison_ positive_negative_tone_resist.svg.

[21] Campo, a. D.; Greiner, C. Journal of Micromechanics and Microengineering 2007, 17, R81-R95.

[22] Abgrall, P.; Conedera, V.; Camon, H.; Gue, A.-M.; Nguyen, N.-T. Electrophoresis 2007, 28, 4539-51.

[23] Zhang, J.; Tan, K. L.; Hong, G. D.; Yang, L. J.; Gong, H. Q. Journal of Micromechanics and Microengineering 2001, 11, 20-26.

[24] Lin, C.-H.; Lee, G.-B.; Chang, B.-W.; Chang, G.-L. Journal of Micromechanics and Microengineering 2002, 12, 590-597.

[25] Zhang, J.; Tan, K.; Gong, H. Polymer Testing 2001, 20, 693-701.

[26] MicroChem, Nano SU-8. 2002; http://www.microchem.com/pdf/SU8_2-25. pdf. 
[27] Keller, S.; Blagoi, G.; Lillemose, M.; Haefliger, D.; Boisen, A. Journal of Micromechanics and Microengineering 2008, 18, 125020.

[28] Cao, C.; Birtwell, S. W.; Hø gberg, J.; Wolff, A.; Morgan, H.; Bang, D. D. Surface Modification of Photoresist SU-8 for Low Autofluorescence and Bioanalytical Applications. 15th International Conference on Miniaturized Systems for Chemistry and Life Sciences. Seattle, Washington, USA, 2011; pp 1161-1163.

[29] Tao, S. L.; Popat, K. C.; Norman, J. J.; Desai, T. A. Langmuir : the ACS journal of surfaces and colloids 2008, 24, 2631-6.

[30] Xue, P.; Bao, J.; Chuah, Y. J.; Menon, N. V.; Zhang, Y.; Kang, Y. Langmuir : the ACS journal of surfaces and colloids 2014, 30, 3110-7.

[31] Hartwig, A.; Schneider, B.; Lühring, A. Polymer 2002, 43, 4243-4250.

[32] Barker, I. a.; Dove, A. P. Triarylsulfonium hexafluorophosphate salts as photoactivated acidic catalysts for ring-opening polymerisation. 2013; http://www.ncbi. nlm.nih.gov/pubmed/24256456.

[33] Dektar, J. L.; Hacker, N. P. Journal of the American Chemical Society 1990, 112, 6004-6015.

[34] Yetisen, A. K.; Akram, M. S.; Lowe, C. R. Lab on a chip 2013, 13, 2210-51.

[35] Lu, Y.; Shi, W.; Jiang, L.; Qin, J.; Lin, B. Electrophoresis 2009, 30, 1497-500.

[36] Carrilho, E.; Martinez, A. W.; Whitesides, G. M. Analytical chemistry 2009, 81, 7091-5.

[37] Martinez, A. W.; Phillips, S. T.; Nie, Z.; Cheng, C.-M.; Carrilho, E.; Wiley, B. J.; Whitesides, G. M. Lab on a chip 2010, 10, 2499-504.

[38] Byrnes, S.; Thiessen, G.; Fu, E. Bioanalysis 2013, 5, 2821-36. 
[39] Chaplan, C. A.; Mitchell, H. T.; Martinez, A. W. Analytical Methods 2014, 6, 1296.

[40] Iyer, P. V.; Ananthanarayan, L. Process Biochemistry 2008, 43, 1019-1032.

[41] Hanefeld, U.; Gardossi, L.; Magner, E. Chemical Society reviews 2009, 38, 453-68.

[42] Mateo, C.; Palomo, J. M.; Fernandez-Lorente, G.; Guisan, J. M.; FernandezLafuente, R. Enzyme and Microbial Technology 2007, 40, 1451-1463.

[43] Haard, N. Food technology 1998, 52, 64-67.

[44] Illanes, A. Electronic Journal of Biotechnology 1999, 2, 1-9.

[45] Vieille, C.; Gregoryzeikus, J. Trends in Biotechnology 1996, 14, 183-190.

[46] Hsiao, K.-t.; Mathur, R.; Advani, S. G.; Gillespie, J. W.; Fink, B. K. Journal of Manufacturing Science and Engineering 2000, 122, 463.

[47] Childs, R. E.; Bardsley, W. G. The Biochemical journal 1975, 145, 93-103.

[48] Kadnikova, E. N.; Kostić, N. M. Journal of Molecular Catalysis B: Enzymatic 2002, $18,39-48$.

[49] Hiner, a. N.; Hernández-Ruíz, J.; Arnao, M. B.; García-Cánovas, F.; Acosta, M. Biotechnology and bioengineering 1996, 50, 655-62.

[50] Walheim, S.; Schaffer, E.; Mlynek, J.; Steiner, U. Science (New York, N.Y.) 1999, 283, $520-2$.

[51] Harvey, D. Modern Analytical Chemistry; McGraw-Hill: Boston, 2000.

[52] Parikh, V. M. Absorption spectroscopy of organic molecules; Addison-Wesley: Massachusetts, 1974.

[53] Deiss, F.; Matochko, W. L.; Govindasamy, N.; Lin, E. Y.; Derda, R. Angewandte Chemie (International ed. in English) 2014, 1-5. 
[54] Chu, P.; Guenther, F.; Lafferty, W. NIST Chemistry WebBook; National Institute of Standards and Technology: Gaithersburg, 1999. 
A. Reference FTIR Spectra 


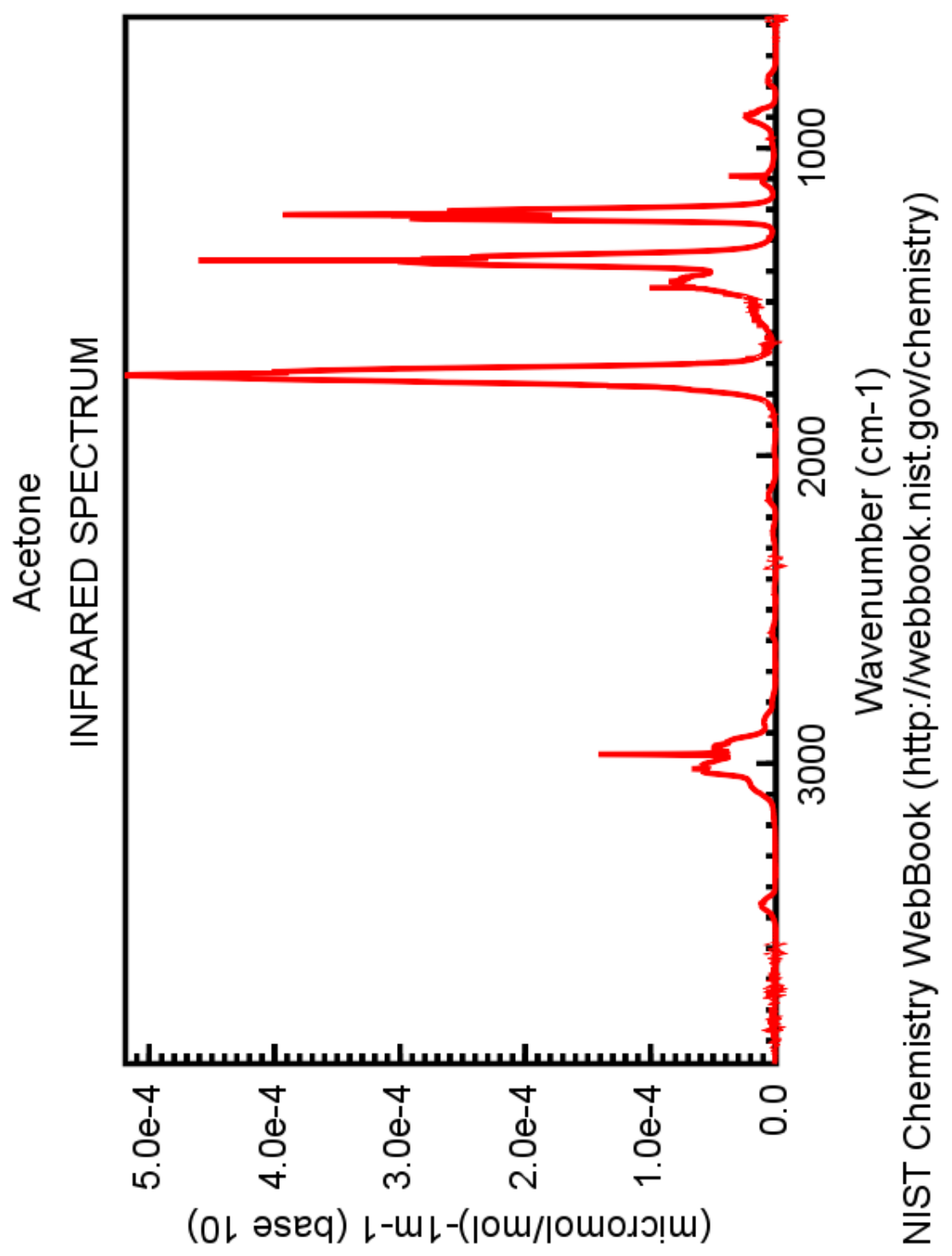

Figure A.1.: FTIR spectrum of acetone. ${ }^{54}$ 


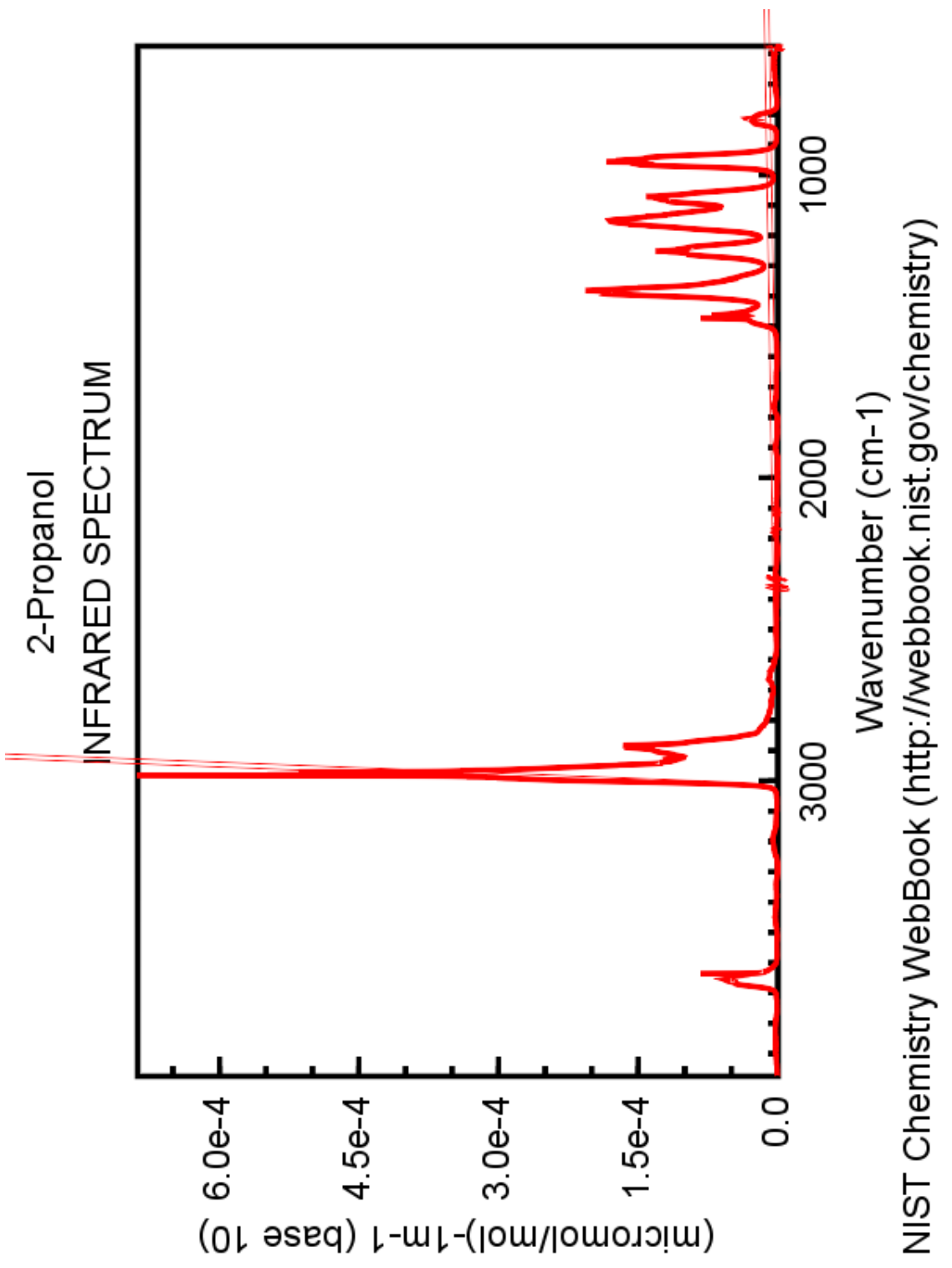

Figure A.2.: FTIR spectrum of 2-propanol. ${ }^{54}$ 
B. Analytical Methods, 2014, 1296 


\section{Paper-based standard addition assays $\uparrow$}

Cite this: DOI: $10.1039 / \mathrm{c} 4 a y 00205 a$

\author{
Cory A. Chaplan, $\$$ Haydn T. Mitchell $\$$ and Andres W. Martinez*
}

Received 22nd January 2014

Accepted 27th January 2014

DOI: $10.1039 /$ c4ay00205a

www.rsc.org/methods

Standard addition assays conducted on paper-based microfluidic devices are introduced as an alternative to external standards for calibrating quantitative tests. To demonstrate this technique, a colorimetric, paper-based, standard addition assay was optimized for the determination of glucose concentrations in the range of 0 to $5 \mathrm{mM}$. Comparable results were obtained from the assay via digital image colorimetry under three different lighting conditions.

In this communication, we introduce standard addition assays as an alternative to external standards for the quantitative detection of analytes on paper-based microfluidic devices (microPADs). Paper-based standard addition assays can be performed in a single sample addition step, and the technique is particularly appealing for quantifying the results of colorimetric assays since it does not require reproducible lighting conditions for digital image colorimetry. We demonstrated the technique by measuring the concentration of glucose in several solutions under three different lighting conditions.

The development of portable, user-friendly and quantitative diagnostic assays is an important part of efforts to improve healthcare in developing countries and remote settings. ${ }^{1}$ Porous membrane-based devices, such as dipsticks, lateral-flow devices and paper-based microfluidic devices, are among the most promising platforms for simple point-of-care diagnostics and typically support colorimetric assays. ${ }^{1-4}$ On their own, colorimetric assays provide only qualitative results, but in combination with a digital camera, colorimetric assays can provide quantitative results via digital image colorimetry (DIC)..$^{5-8}$

In DIC, the concentration of analytes is determined by taking a digital picture of a concluded colorimetric assay, reading the intensity of the color in the image, and then comparing the intensity to an external calibration curve. ${ }^{8}$ This technique is

Department of Chemistry \& Biochemistry, California Polytechnic State University, San Luis Obispo, CA 93401, USA. E-mail: awmartin@calpoly.edu

$\dagger$ Electronic supplementary information (ESI) available. See DOI: 10.1039/c4ay00205a

† These authors contributed equally to this project. appealing for the quantification of analytes in the field because it can be performed using a digital camera, ${ }^{6}$ it is compatible with porous-membrane-based diagnostic devices, ${ }^{8}$ and colorimetric assays already exist for a large number of analytes. ${ }^{9}$ The problem with DIC, in the context of point-of-care testing, is that the intensities of the colors in a digital image vary with the lighting conditions under which the image is obtained. ${ }^{10}$ Therefore, if an assay is imaged under one set of lighting conditions in the field, and the color intensity is compared to an external calibration curve that was prepared in a laboratory under a different set of lighting conditions, then the results would not be accurate..$^{8,10}$

Three different approaches have been described for overcoming the lighting intensity problem associated with DIC: (i) controlling the lighting conditions in the field by using a portable scanner, a special light, or a light-box;, ${ }^{411-13}$ (ii) analyzing a series of external standard solutions in parallel with the unknown, and imaging all the results at the same time under the same lighting conditions; and (iii) imaging the device alongside a color calibration chart, and using the calibration chart and an associated algorithm to compensate for variations in lighting conditions. ${ }^{10}$ The first two approaches are inconvenient for point-of-care diagnostics since they require additional equipment, reagents and time. The third approach was studied in detail by Murdock et al., and they concluded that color calibration charts would not allow for accurate calibration of colorimetric assays that produce varying intensities of the same color, and that a series of external standard solutions would most likely still need to be analyzed and imaged in parallel with the sample in order to account for variations in lighting conditions. ${ }^{14}$ Ultimately, all three approaches rely on an external calibration curve and are not ideal for point-of-care colorimetric assays.

The method of standard additions provides an alternative to external standard calibration. Standard addition assays are used in quantitative analysis to overcome matrix effects that can affect the signal of an assay. ${ }^{15}$ In a traditional single-point standard addition assay, the signal of an unknown sample is 
measured, and then, the signal of the unknown plus a spike of analyte of known concentration is measured. From these data, one can calculate the concentration of the unknown sample without relying on an external calibration curve. ${ }^{16}$ Since standard addition assays do not rely on an external calibration curve, the results are not affected by variations in lighting conditions. While traditional standard addition assays are timeconsuming since several solutions must be prepared and tested for each sample to be analyzed, ${ }^{15}$ multiple standard addition assays can be performed simultaneously on a microPAD from a single sample-addition step. The reagents and the spike of analyte can both be added to the device during fabrication, stored on-chip and then re-suspended in the sample once the sample is added to the device (Fig. 1). ${ }^{17}$ This approach allows for spikes of analyte to be added to unknown samples automatically, without any additional input from the user and without diluting the sample to any significant extent.

We chose glucose as the analyte to demonstrate the technique for two principal reasons: (i) the measurement of glucose by DIC on paper-based devices using external calibration curves has been well characterized, ${ }^{8}$ and (ii) glucose can be dried in a channel of a paper-based microfluidic device and will dissolve in a sample solution as the solution wicks along the channel, ${ }^{17}$ which is a requirement for this particular form of paper-based standard addition assay. We optimized an enzymatic assay for glucose using 2,2'-azino-bis(3-ethylbenzothiazoline-6-sulfonic acid) diammonium salt (ABTS), which produced a blue-green colored product when glucose was present in the sample. ${ }^{19,20}$ The standard addition assay was optimized to determine glucose concentrations in the range of 0 to $5 \mathrm{mM}$, since glucose levels in urine above $1.4 \mathrm{mM}$ can be indicative of disease. ${ }^{18}$

We fabricated paper-based microfluidic devices by wax printing with a central sample zone from which the sample was distributed through eight channels (spokes) into eight test zones (Fig. 1). ${ }^{21}$ The device was designed to run four replicates of sample $\left(S_{0}\right)$ and four replicates of sample spiked with a known concentration of glucose $\left(S_{1}\right)$. A reagent mixture containing $25 \mathrm{mM}$ ABTS, $75 \mathrm{kU} \mathrm{L}^{-1}$ glucose oxidase, $250 \mathrm{kU} \mathrm{L}^{-1}$ horseradish peroxidase and $1 \mathrm{M}$ trehalose dissolved in $1 \times$ PBS

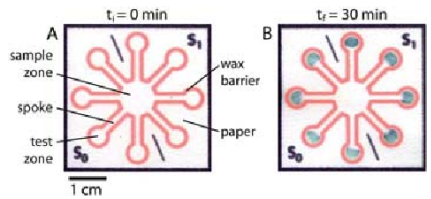

Fig. 1 Paper-based standard addition devices. (A) A device before addition of the sample. Reagents for the colorimetric glucose assay were dried in each test zone. A glucose spike solution was dried in the four spokes on the side of the device labeled $S_{1}$. (B) A device 30 minutes after adding a sample containing $2.5 \mathrm{mM}$ glucose to the sample zone. When the sample is added to the device, it wicks along the spokes dissolving any dried glucose and transporting it into the test zones. The intensity of the color in the $S_{1}$ test zones is always greater than that in the $S_{0}$ test zones because of the additional glucose from the spokes. was added to each test zone, and a $5.0 \mathrm{mM}$ glucose spike solution was added to the spokes of the $S_{1}$ sample zones. We then tested a series of glucose solutions prepared in $1 \times$ PBS by adding $35 \mu \mathrm{L}$ of the sample solution to the sample zone. No other action from the user was required, and the assays were complete after 30 minutes (please see the ESI for a discussion of the experimental design and additional experimental details $\dagger$ ).

To demonstrate the capability of the standard addition assay to provide quantitative results via DIC that were independent of lighting conditions, the devices were imaged under three different lighting conditions: (i) scanner with an LED light source, (ii) digital camera outdoors in sunlight and (iii) digital camera indoors under fluorescent lighting. The color intensities of the test zones in the red channel of the digital images were read using Image J.

Since the intensity of the color of paper-based colorimetric assays is usually not directly proportional to the concentration of analyte, ${ }^{\mathbf{8}}$ a non-linear standard addition assay was developed. We chose a rectangular hyperbolic equation as the basis for the non-linear standard addition calculations because this equation described accurately the relationship between signal and concentration for the glucose assay (Fig. 2), and it allowed for the derivation of an equation relating the concentration of glucose to the signal from the assay. The signal from the assay $(S)$ can be described as:

$$
S=\frac{S_{\max } C}{K+C}
$$

where $S_{\max }$ is the maximum signal (color intensity) that can be achieved, $C$ is the concentration of analyte, and $K$ is a constant equal to the concentration of analyte at which half of the maximum signal is achieved. Rewriting eqn (1) in the context of a single-point standard addition assay yields two equations:

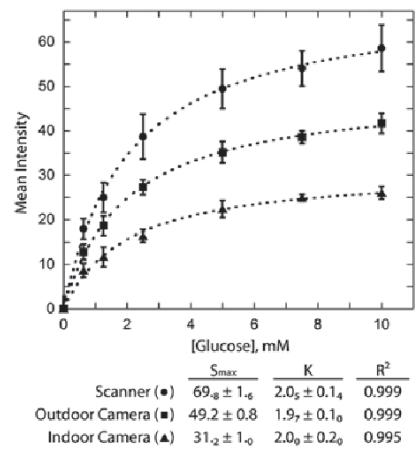

Fig. 2 External calibration curves for the glucose assay under three lighting conditions: scanner (O), outdoor camera ( $\mathbf{a}$ ) and indoor camera ( $\mathbf{\Delta}$ ). Data points represent the mean of eight trials, and error bars represent one standard deviation from the mean. The curves were fit with eqn (1), and values for $S_{\max }, K$ and $R^{2}$ were obtained using KaleidaGraph®. 


$$
\begin{gathered}
S_{0}=\frac{S_{\max } C_{\mathrm{u}}}{K+C_{\mathrm{u}}} \\
S_{1}=\frac{S_{\max }\left(C_{\mathrm{u}}+C_{\mathrm{s}}\right)}{K+\left(C_{\mathrm{u}}+C_{\mathrm{s}}\right)}
\end{gathered}
$$

where $S_{0}$ is the signal from the unknown sample, $S_{1}$ is the signal from the unknown sample plus the spike of analyte, $C_{\mathrm{u}}$ is the concentration of the unknown, and $C_{\mathrm{s}}$ is the concentration of the spike. From eqn (2) and (3), we can solve for $C_{\mathrm{u}}$ in terms of $S_{0}, S_{1}, C_{\mathrm{s}}$, and either $S_{\max }$ or $K$. Since the intensities of color are known to vary with lighting conditions, we chose to solve for $C_{\mathrm{u}}$ in terms of $K$ under the hypothesis that the magnitude of $K$ would not vary with lighting conditions. The concentration of the unknown can thus be solved as:

$$
C_{\mathrm{u}}=\sqrt{\frac{\left(C_{\mathrm{s}}+K\right)^{2}}{4}+\frac{S_{0} C_{\mathrm{s}} K}{S_{1}-S_{0}}}-\frac{C_{\mathrm{s}}+K}{2}
$$

Eqn 4 was used to calculate the concentration of glucose in samples. The values for $S_{1}$ and $S_{0}$ came from the respective means of the color intensities of the four $S_{1}$ and $S_{0}$ test zones from each device. The value for $K$ was obtained from an external calibration curve that was fit with eqn (1) (Fig. 2), and the value for $C_{\mathrm{s}}$ was determined experimentally by testing known glucose solutions using devices with a spike of glucose in all eight spokes (Fig. 3).

The external calibration curves for the three different lighting conditions are shown in Fig. 2. All three curves have the same general shape, and fitting the curves with eqn (1) provided excellent fits, giving correlation values $\left(R^{2}\right)$ of $0.999,0.999$ and 0.995 for the scanner, outdoor camera and indoor camera respectively. As expected, the values of $S_{\max }$ varied significantly with lighting conditions. The value for $K$, on the other hand, was constant for all three lighting conditions with an average value of $2.0 \mathrm{mM}$. This result was critical to the success of this project and confirmed the initial hypothesis that the magnitude of $K$ would not be affected by lighting conditions.

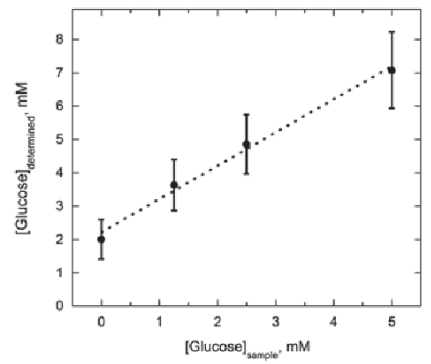

Fig. 3 Glucose spike calibration curve. Concentrations from each trial were determined using the scanner's external calibration curve. Each data point represents the mean of eight trials, and error bars represent one standard deviation from the mean. Linear regression analysis of the data gave a slope of $1.00 \pm 0.06$, an intercept of $2.2 \pm 0.2$ and an $R^{2}$ value of 0.992 for the best-fit line. The magnitude of the intercept represents the concentration of the spike $\left(C_{s}\right)$.
A second critical result was that the amount of glucose "spiked" into the sample from the spoke was constant for the range of glucose concentrations that were tested (Fig. 3). The average concentration of the spike $\left(C_{\mathrm{s}}\right)$ was $2.2 \mathrm{mM}$. This concentration was lower than the concentration of the $5.00 \mathrm{mM}$ glucose spike solution that was added to the spokes, which could be due to one or two reasons: (i) the glucose in the spoke did not dissolve completely in the sample, so less than half of the glucose added to the spoke actually reached the test zone, or (ii) the glucose dried in the spoke was diluted compared to its initial concentration. Ultimately, the source of the difference in concentrations is irrelevant to the results of the standard addition assay.

Using a value of $2.0 \mathrm{mM}$ for $\mathrm{K}$, a value of $2.2 \mathrm{mM}$ for $C_{\mathrm{s}}$ and the average values for $S_{1}$ and $S_{0}$ obtained from the scanned images for each device, the concentration of glucose in five different samples were determined using eqn (4) (Table 1). These results illustrate the accuracy and precision of the standard addition assay. While a large relative range was observed for the three trials of each sample, the mean value was always within one standard deviation of the true value. These results are typical of paper-based colorimetric assays, which tend to display a low level of precision, but relatively good accuracy when multiple trials are averaged to minimize the effects of indeterminate error. ${ }^{8}$ Using a 3D-microPAD, ${ }^{22,23}$ it should be possible to run dozens or even hundreds of replicates for $S_{0}$ and $S_{1}$ simultaneously on a single device, which should further improve the accuracy of the results. It should also be possible to perform multi-point standard addition assays by spiking the sample with different concentrations of analyte, which could also improve the accuracy of the results. The precision of the assays could likely be improved by using an automated liquid dispensing system to deposit the reagents on the devices.

The results from the three different lighting conditions are shown in Fig. 4 and Table 2. The results from the standard addition assays retain the same level of accuracy under different lighting conditions, while the accuracy of the results from the external calibration assays decreases significantly when the lighting conditions of the external standards do not match the lighting conditions of the samples (Fig. 4, Table S1 $\dagger$ ). A $t$-test confirmed there was no statistically significant difference between the results from the external calibration assay with controlled lighting conditions (scanner) and the standard

Table 1 Results from standard addition assays imaged with a scanner

\begin{tabular}{lclcc}
\hline Sample & $\begin{array}{l}\text { Trial 1 } \\
(\mathrm{mM})\end{array}$ & $\begin{array}{l}\text { Trial 2 } \\
(\mathrm{mM})\end{array}$ & $\begin{array}{l}\text { Trial 3 } \\
(\mathrm{mM})\end{array}$ & $\begin{array}{l}\text { Mean } \pm \text { std } \\
\text { dev. (mM) }\end{array}$ \\
\hline $0 \mathrm{mM}$ & -0.03 & 0.02 & -0.06 & $-0.02 \pm 0.04$ \\
$1.25 \mathrm{mM}$ & 1.49 & 1.03 & 1.46 & $1.3 \pm 0.3$ \\
$2.50 \mathrm{mM}$ & 2.34 & 2.63 & 2.12 & $2.4 \pm 0.3$ \\
$5.00 \mathrm{mM}$ & 4.72 & 5.24 & 4.84 & $4.9 \pm 0.3$ \\
Artificial urine $^{a}$ & 2.49 & 1.89 & 2.23 & $2.2 \pm 0.3$ \\
Unknown $^{b}$ & 2.81 & 2.40 & 2.19 & $2.5 \pm 0.3$
\end{tabular}

a Contained $2.5 \mathrm{mM}$ glucose and $5 \mathrm{mM}$ tartrazine, a yellow dye, to simulate the color of urine. ${ }^{b}$ Contained $2.5 \mathrm{mM}$ glucose and was analyzed in a blind experiment. 


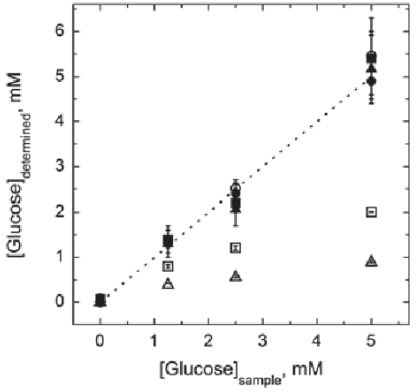

Fig. 4 Comparison of the standard addition assay results (solid shapes) to external calibration results (open shapes) for the three

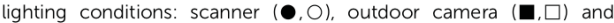
indoor camera $(\boldsymbol{\Lambda}, \Delta)$. The external calibration results for all three lighting conditions were calculated using the scanner's external calibration curve. Data points represent the mean of three trials, and error bars represent one standard deviation from the mean. The dashed line represents the desired result where the determined concentration equals the actual concentration of glucose in the sample.

Table 2 Results from the standard addition assays determined for the three lighting conditions. External calibration results for the scanner data are also shown for comparison. Results are all reported as the mean \pm standard deviation for three trials

\begin{tabular}{lcccc}
\hline Sample & $\begin{array}{l}\text { Scanner } \\
(\mathrm{mM})\end{array}$ & $\begin{array}{l}\text { Outdoor } \\
\text { camera }(\mathrm{mM})\end{array}$ & $\begin{array}{l}\text { Indoor } \\
\text { camera }(\mathrm{mM})\end{array}$ & $\begin{array}{l}\text { Scanner } \\
\text { ext. cal. }(\mathrm{mM})\end{array}$ \\
\hline $0 \mathrm{mM}$ & $-0.02 \pm 0.04$ & $0.07 \pm 0.03$ & $0.10 \pm 0.07$ & $-0.02 \pm 0.03$ \\
$1.25 \mathrm{mM}$ & $1.3 \pm 0.3$ & $1.4 \pm 0.3$ & $1.3 \pm 0.1$ & $1.31 \pm 0.09$ \\
$2.50 \mathrm{mM}$ & $2.4 \pm 0.3$ & $2.1 \pm 0.2$ & $2.1 \pm 0.4$ & $2.5 \pm 0.1$ \\
$5.00 \mathrm{mM}$ & $4.9 \pm 0.3$ & $5.4 \pm 0.9$ & $5.2 \pm 0.8$ & $5.4 \pm 0.5$ \\
Art. urine $^{a}$ & $2.2 \pm 0.3$ & $2.4 \pm 0.5$ & $2.2 \pm 0.3$ & $2.58 \pm 0.07$ \\
Unknown $^{b}$ & $2.5 \pm 0.3$ & $2.2 \pm 0.5$ & $2.1 \pm 0.6$ & $2.8 \pm 0.2$
\end{tabular}

${ }^{a}$ Contained $2.5 \mathrm{mM}$ glucose and $5 \mathrm{mM}$ tartrazine, a yellow dye, to simulate the color of urine. ${ }^{b}$ Contained $2.5 \mathrm{mM}$ glucose and was analyzed in a blind experiment.

addition assays under all three lighting conditions at the $95 \%$ confidence level. The assay is most precise at lower concentrations of glucose where the differences between $S_{1}$ and $S_{0}$ are largest. At higher concentrations of glucose, as the difference between $S_{1}$ and $S_{0}$ decreases, the precision of the results tends to decrease. It should be possible to tune the range of concentrations of glucose that can be detected using this method by changing the concentration of glucose in the spike.

We demonstrated a paper-based, colorimetric, standard addition assay for glucose that is simple to perform and provides quantitative results by DIC that are independent of lighting conditions. These advantages are important in the context of point-of-care diagnostics and telemedicine, especially since lighting conditions in the field are difficult to control. In its present form, the paper-based standard addition assay can only be used for analytes that can be dried on a device, stored and then re-suspended in a sample, but the technique is not limited to colorimetric assays. Paper-based standard additions could be adapted to any type of paper-based assay including fluorescence assays, absorbance assays, electrochemical assays and chronometric assays. ${ }^{24-30}$ The quantitative results of these assays are not typically affected by ambient lighting conditions, but the method of standard additions could still be useful for overcoming matrix effects and for calibrating devices in the field without the need for a standard reference solution.

\section{Acknowledgements}

This project was supported by a Non-Tenured Faculty Grant from $3 \mathrm{M}$, the Extramural Funding Initiative (EFI) at California Polytechnic State University, San Luis Obispo, and the California State University Program for Education and Research in Biotechnology (CSUPERB). We would like to thank Emily Fogle for help with fitting the calibration curves in Kaleidgraph.

\section{References}

1 C. D. Chin, V. Linder and S. K. Sia, Lab Chip, 2007, 7, 41-57. 2 P. von Lode, Clin. Biochem., 2005, 38, 591-606.

3 A. W. Martinez, S. T. Phillips, M. J. Butte and G. M. Whitesides, Angew. Chem., Int. Ed., 2007, 46, 13181320.

4 A. K. Yetisen, M. S. Akram and C. R. Lowe, Lab Chip, 2013, 13, 2210-2251.

5 W. Colin, E. Mark, M. Bonner and R. S. Pomeroy, Trends Anal. Chem., 1993, 12, 395-403.

6 L. Byrne, J. Barker, G. Pennarun-Thomas and D. Diamond, Trends Anal. Chem., 2000, 19, 517-522.

7 A. Lopez-Molinero, D. Liñan, D. Sipiera and R. Falcon, Microchem. J., 2010, 96, 380-385.

8 A. W. Martinez, S. T. Phillips, E. Carrilho, S. W. Thomas, H. Sindi and G. M. Whitesides, Anal. Chem., 2008, 80, 3699-3707.

9 E. Jungreis, in Spot Test Analysis: Clinical, Environmental, Forensic, and Geochemical Applications, 2nd edn, John Wiley \& Sons, Inc., New York, 1997.

10 L. Shen, J. A. Hagen and I. Papautsky, Lab Chip, 2012, 12, 4240-4243.

11 O. Mudanyali, S. Dimitrov, U. Sikora, S. Padmanabhan, I. Navruz and A. Ozcan, Lab Chip, 2012, 12, 2678-2686.

$12 u$ Chek, Biosense Technologies Pvt. Ltd, http://www.uchek.in.

13 D. Lee, B. G. Jeon, C. Ihm, J. Park and M. Y. Jung, Lab Chip, 2011, 11, 120-126.

14 R. C. Murdock, L. Shen, D. K. Griffin, N. Kelley-Loughnane, I. Paputsky and J. A. Hagen, Anal. Chem., 2013, DOI: 10.1021/ac403040a, just accepted manuscript.

15 D. Harvey, in Analytical Chemistry 2.0, published online at http://www.asdlib.org/onlineArticles/ecourseware/Welcome. htmlhttp://www.asdlib.org/onlineArticles/ecourseware/Wel come.html, 2008, pp. 161-167.

16 The relationship between signal and concentration must be known, and standard addition assays are typically 
performed for assays with a linear relationship between signal and concentration.

17 K. C. Schilling, D. Jauregui and A. W. Martinez, Lab Chip, 2013, 13, 628-631.

18 J. K. Davidson, in Clinical Diabetes Mellitus: A ProblemOriented Approach, 3rd edn, Thieme, New York, 2000, p. 444.

19 D. R. Nelson and A. K. Huggins, Anal. Biochem., 1974, 59, 46-53.

20 R. C. Bateman and J. A. Evans, J. Chem. Educ., 1995, 72, A240A241.

21 E. Carrilho, A. W. Martinez and G. M. Whitesides, Anal. Chem., 2009, 81, 7091-7095.

22 A. W. Martinez, S. T. Phillips and G. M. Whitesides, Proc. Natl. Acad. Sci. U. S. A., 2008, 105, 19606-19611.

23 G. G. Lewis, M. J. DiTucci, M. S. Baker and S. T. Phillips, Lab Chip, 2012, 12, 2630-2633.
24 E. Carrilho, S. T. Phillips, S. J. Vella, A. W. Martinez and G. M. Whitesides, Anal. Chem., 2009, 81, 5990-5998.

25 N. K. Thom, K. Yeung, M. B. Pillion and S. T. Phillips, Lab Chip, 2012, 12, 1768-1770.

26 A. K. Ellerbee, S. T. Phillips, A. C. Siegel, K. A. Mirica, A. W. Martinez, P. Striehl, N. Jain, M. Prentiss and G. M. Whitesides, Anal. Chem., 2009, 81, 8447-8452.

27 W. Dungchai, O. Chailapakul and C. S. Henry, Anal. Chem., 2009, 81, 5821-5826.

28 Z. Nie, C. A. Nijhuis, J. Gong, X. Chen, A. Kumachev, A. W. Martinez, M. Narovlyansky and G. M. Whitesides, Lab Chip, 2010, 10, 477-483.

29 J. P. Metters, S. M. Houssein, D. K. Kampouris and C. E. Banks, Anal. Methods, 2013, 5, 103-110.

30 G. G. Lewis, M. J. DiTucci and S. T. Phillips, Angew. Chem., Int. Ed., 2012, 51, 12707-12710. 